\title{
Study of the hydrogen escape rate at Mars during Martian years 28 and 29 from comparisons between SPICAM/Mars Express observations and GCM-LMD simulations
}

\author{
Chaufray, J-Y ${ }^{1}$., F. Gonzalez-Galindo 2 , M.A. Lopez-Valverde 2 , F. Forget ${ }^{3}$, E. Quémerais ${ }^{1}$, J-L. Bertaux ${ }^{1}$, F. \\ Montmessin ${ }^{1}$, M. Chaffin ${ }^{4}$, N. Schneider ${ }^{4}$, J.T. Clarke ${ }^{5}$, F. Leblanc ${ }^{1}$, R. Modolo ${ }^{1}$, R.V. Yelle ${ }^{6}$ \\ ${ }^{1}$ LATMOS,CNRS, Guyancourt, France, ${ }^{2} I A A$, CSIC, Granada, Spain, ${ }^{3} L M D, C N R S$, Paris, France, ${ }^{4} L A S P$, \\ Boulder, CO, USA, ${ }^{5}$ Boston University, Boston, MA, USA, ${ }^{6}$ University of Arizona, Tucson, AZ, USA
}

Abstract

We have simulated the 3D atomic hydrogen density in the Martian upper atmosphere and associated Jeans escape rate during Martian years 28 and 29. The coronal Lyman- $\alpha$ brightness is computed using a 3D radiative transfer model which accounts for the monthly average hydrogen density for these two years and is compared to a large set of observations by Mars Express/SPICAM. The simulated brightness is generally in good agreement with the observations for $\mathrm{Ls}<230^{\circ}$ and Ls $>330^{\circ}$ for Martian year 28 and Ls $<270^{\circ}$, Ls $>340^{\circ}$ for Martian year 29 , but the model strongly underestimated the brightness for $230<\mathrm{Ls}<330^{\circ}$ for Martian year 28 and $270<\mathrm{Ls}<340^{\circ}$ for Martian year 29. In these simulations the transport of water vapor contributes to the production of hydrogen at high altitudes during southern summer. A possible explanation for the model discrepancy is an underestimate of this water transport, associated with an underestimate of the hygropause altitude and/or an underestimate of the supersaturation of the mesosphere. Considering this discrepancy, we estimate the hydrogen escape rate during these two Martian years to vary by almost two orders of magnitude, between $\sim 10^{25}$ to $6 \times 10^{26} \mathrm{~s}^{-1}$ (equivalent to a global layer of water $\sim 33$ to $2000 \mathrm{~mm}$ deep every billion years), in agreement with the seasonal variations estimated directly from the fit of the SPICAM observations during the Martian year 28 by Chaffin et al. (2014). Our analysis suggests that episodic dust storms and associated enhancements at high altitude near perihelion are a major factor in the $\mathrm{H}$ escape estimates averaged over one martian year or longer periods, but the accumulated water lost at this rate for 4 billions years is much lower than the amount of water needed to form the flow channels observed on Mars.

1) Introduction

Several geologic and mineralogic observations indicate that Mars was not always as dry as it is today (e.g. Bibring et al. 2006, Bishop et al. 2008, Hynek et al. 2010). The fate of the water is still unknown, but substantial amounts could have escaped into the interplanetary medium in the form of atomic hydrogen (Jakosky et al. 2018). So, understanding 
of how water currently escapes from Mars is necessary to investigate the long-term evolution of the Martian climate.

Recent observations suggest that the amount of exospheric hydrogen at Mars has important seasonal variations, with a larger abundance during southern summer (Chaffin et al. 2014, Clarke et al. 2014, 2017, Bhattacharyya et al. 2015). This increase of the hydrogen density should be associated with an increase of the hydrogen Jeans escape. These seasonal variations have a large impact on the plasma environment of Mars (Bertucci et al. 2013, Yamauchi et al. 2015, Romanelli et al. 2015, Halekas 2017, Rahmati et al. 2018), and could be driven by large amounts of water vapor in the mesosphere, as observed by Mars Express/SPICAM (Maltagliati et al. 2017, Fedorova et al. 2018), during dust storm season (Chaffin et al. 2017, Heavens et al. 2018). Using the Global Circulation Model of Laboratoire de Météorologie Dynamique (GCM-LMD) (Forget et al. 1999, Gonzalez-Galindo et al. 2009, 2015), we were able to simulate seasonal variations of the hydrogen escape, but with a lower range than derived from observations (Chaufray et al. 2015a). In order to better characterize the hydrogen exosphere of Mars and the variability of hydrogen escape, we performed a detailed study of the Martian years 28 and 29, and compared the simulated brightness to a large SPICAM Lyman- $\alpha$ dataset during the same two years. The year number used in this study follows the convention chosen by Clancy et al. (2000) with April $111955\left(\mathrm{Ls}=0^{\circ}\right)$ chosen as the beginning of the Martian year 1.

The data used in this study is described in section 2. In section 3, we present our 3D simulated Lyman- $\alpha$ emissions corresponding to the Martian years 28 and 29, which are obtained by coupling several models. The comparisons between the observed and simulated Lyman- $\alpha$ profiles are presented in section 4 and the possible origin of the discrepancy is discussed in section 5 , followed by conclusions in the last section.

\section{2) Observations}

The Spectroscopy for Investigation and Characterization of the Atmosphere of Mars (SPICAM) is a dual ultraviolet and infrared spectrometer aboard Mars Express (Bertaux et al. 2006, Montmessin et al. 2017). The ultraviolet channel uses an optical entrance of $4 \mathrm{~cm}$ diameter feeding an off-axis parabolic mirror which focuses the observed scene at the focal plane. The instantaneous field of view (IFOV) is limited at the focal point of the parabolic mirror by a 50 $\mu \mathrm{m}$ wide retractable slit that extends over an angular aperture of $2.8^{\circ}$, equivalent to about two pixels on the UV detector. In the upper most portion of the slit, a 10 times wider aperture allows for the observations of fainter sources at the expense of degraded spectral resolution. 
In principle, SPICAM can record 288 spatially resolved spectra along the slit. However, to save data volume transmission, only 5 spectra are transmitted each second. For all the observations presented here, the spectra are the sum of 32 individual CCD line spectra forming 5 adjacent spatial bins of $0.32^{\circ}$ each, starting from the CCD line $\mathrm{Y}_{0}$. The wavelength range of the UV channel is $118-320 \mathrm{~nm}$ and includes the strong hydrogen Lyman- $\alpha$ emission line at $121.6 \mathrm{~nm}$. This emission line is produced by resonant scattering of solar photons by hydrogen atoms in the Martian upper atmosphere. Such emission is observed over several Martian radii as a signature of the extended hydrogen exosphere of Mars (Chaufray et al. 2008).

We selected several observations obtained during the Martian years 28 (from 22 Jan 2006 to 9 Dec 2007) and 29 (from 10 Dec 2007 to 26 Oct 2009) performed by the UV spectrograph SPICAM on Mars Express, including those studied by Chaffin et al. (2014). During this period the UV channel exhibited an anomalous image intensifier behavior, leading to sporadic changes of the high voltage during a sequence of observations (Montmessin et al. 2017). We selected by visual inspection the observations least affected by this behavior. A cleaning procedure has been developed to flag the UV channel spectra affected by these high voltage spikes (Montmessin et al. 2017). All these flagged spectra have been removed from use in this study.

The list of observations used for this study is summarized in Tables 1 and 2 in theAppendix.

Some of the observations were performed without the slit, while others were performed with the slit. Also, the 5 CCD spatial bands_(corresponding to a binning of 32 individual, consecutive lines) transmitted each second can differ between observations. Three different configurations are used in the observations presented here. The first configuration corresponds to an observation with the slit and starts from $\mathrm{CCD}$ line $\mathrm{Y}_{0}=23$, the second configuration also corresponds to an observation with the slit, but starts from CCD line $\mathrm{Y}_{0}=118$, and the third configuration corresponds to an observation without the slit, starting from CCD line $\mathrm{Y}_{0}=118$.

For the first configuration, all the transmitted bands correspond to the part of the CCD in front of the narrow part of the slit, so we chose band 5, which is close to the center of the CCD (lines 118- 149). For the second configuration, the last two bands correspond to the part of the CCD in front of the large part of the slit. The signal to noise ratio is generally better, and straylight near 132 and $143 \mathrm{~nm}$ discussed by Leblanc et al. (2006) and Chaufray et al. (2009) is not detected in those bands. Therefore, we chose band 4 (lines 214-245), which is more sensitive than band 5 by about $20 \%$ (Fig. 1a). For the last configuration without the slit, we chose band 1, which corresponds to the same CCD lines as configuration 1 (lines 118-149), but is slightly different (by about 5\%) than the derived brightness from band 

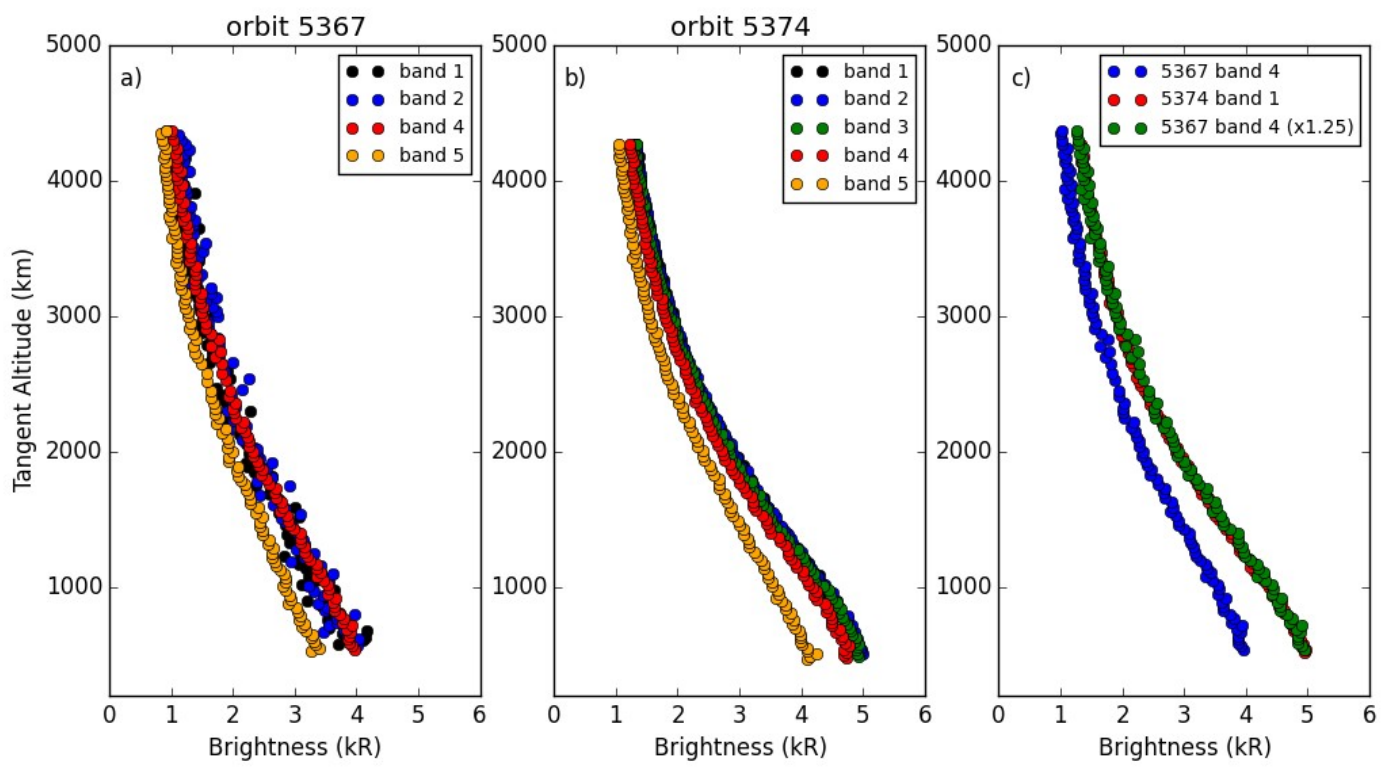

4 (lines 214-245) (Fig. 1b). As shown in Fig. 1, the signal to noise ratio of the derived brightness profile is very good and trying to improve it by using the 5 transmitted bands will not change our results since the calibration uncertainty is larger than the standard deviation of the measurement.

Fig. 1 : a) Brightness profiles derived from Mars Express orbit 5367 with the slit over the first 2 and last 2 transmitted bands of the CCD. b) Brightness profiles derived from Mars Express orbit 5374 without the slit over the 5 transmitted bands. c) Comparison between the profiles derived from the two orbits over the bands used in this study considering a 1.25 factor for the observations with the slit

Observations with very similar geometries (orbits 5367 and 5374 for example, Fig. 1) obtained with and without the slit, indicate a systematic $\sim 20 \%$ underestimate of the derived brightness with the slit compared to the observations without the slit (Fig. 1b). 5\% could be attributed to the different CCD lines as suggested by Fig. 1b. The rest of the difference could come from the method used to integrate the spectral line, neglecting the extended spectral wings, or in a continuum background subtraction. Therefore, to summarize, the brightness measured by configuration 3 is used as a reference, and a correction by $20 \%$ is applied to the brightness measured in configuration 1 and a correction of $25 \%$ is applied to the brightness measured by configuration 2. Examples of Lyman- $\alpha$ vertical profiles are displayed in Fig. 1.

The spectral shape of the Lyman- $\alpha$ line measured for each configuration is displayed in Fig. 2. For the first two configurations, the brightness is computed by integration over the line profile after subtraction of a residual linear background (Chaufray et al. 2008). For the third (slitless) configuration, the average number of counts per pixel is computed and converted to Rayleighs using the solid angle defined by one pixel. All spectra have been corrected for 


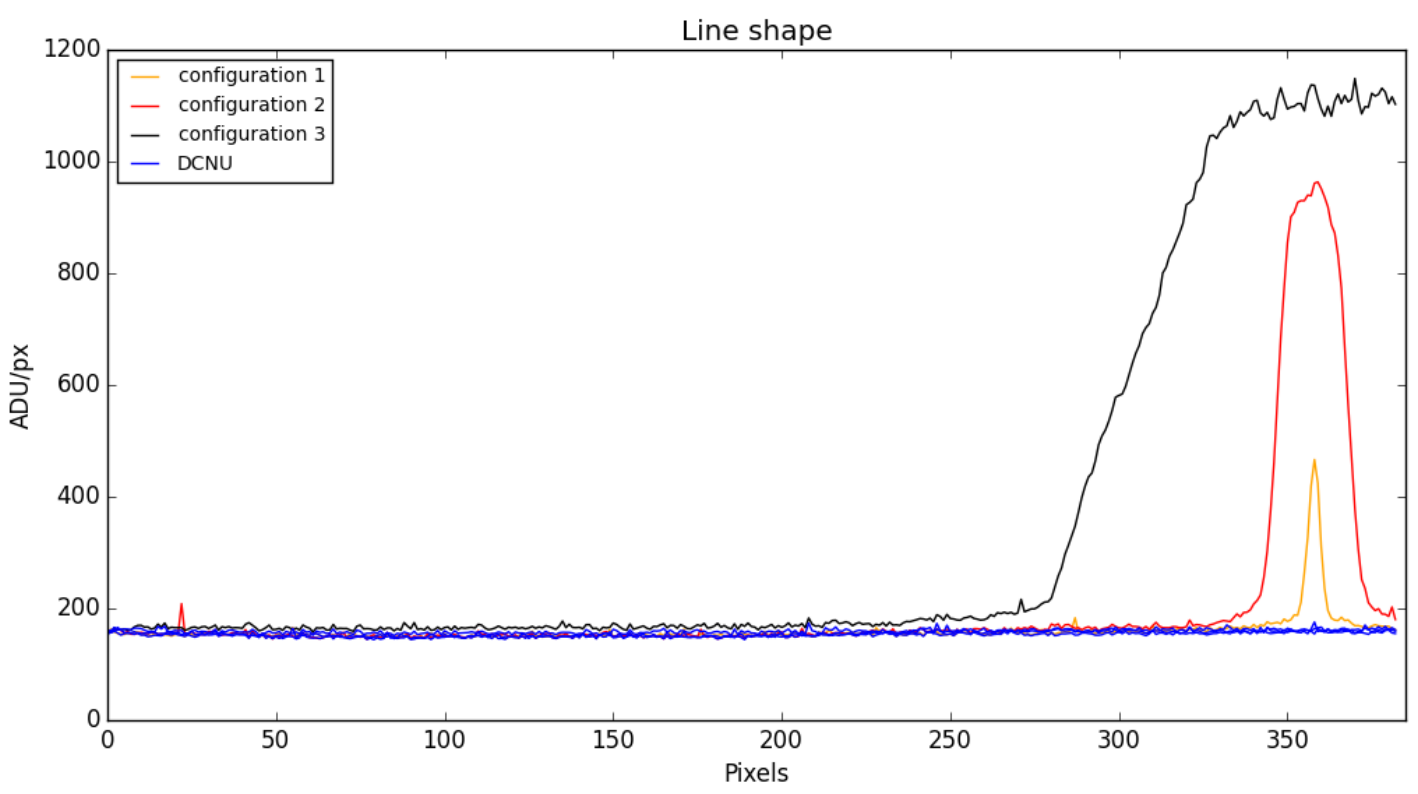

Fig. 2 Spectral shape of the Lyman-a line measured for the three configuration used in the paper. These spectral profile correspond to the average observation between 2000 and $2200 \mathrm{~km}$ from observation 5367 (narrow slit : configuration 1 and large slit : configuration 2) and observation 5374 (no slit : configuration 3). A sapphire filter is glued above the CCD MgF 2 input window, preventing Lyman- $\alpha$ photons to reach pixels $<250$ without slit (Bertaux et al. 2006). With the slit, the linewidth is constrained by the slit size. The Dark charge non-uniformity (DCNU) is also indicated in blue and is very similar for these three measured spectra.

Such a systematic uncertainty is in the range of the absolute calibration of the instrument from star observations (Montmessin et al. 2017). Therefore, in this study we focus on systematic differences between the observations and the simulations which are $>25 \%$, as well as the differences in the shape of the observed and simulated vertical variations.

\section{3) Models}

To simulate the hydrogen corona of Mars during Martian years 28 and 29, we used three models. The first model is the GCM-LMD describing the Martian atmosphere from the surface to the exobase (Forget et al. 1999, GonzalezGalindo et al. 2009, 2015), including the hydrogen in the thermosphere (Chaufray et al. 2015a, 2018). The second model is an exospheric model considering nonuniform conditions at the exobase (hydrogen density and temperature from the GCM-LMD) to derive the hydrogen density in the exosphere, based on the approach of Vidal-Madjar and Bertaux 1972. The last model is a 3D Monte Carlo radiative transfer model used to simulate the resonance Lyman- $\alpha$ 
spectral volume emission rate (Chaufray et al 2015b). The Lyman- $\alpha$ brightness is computed by formal integration of the radiative transfer equation using simulated volume emission rates for the specific geometry (spacecraft position and line of sight direction) of each individual observation listed in Tables 1 and 2 in Appendix A. The simulated brightness is directly proportional to the solar flux at the center of the solar Lyman- $\alpha$ line. To derive it, we used the solar brightness between 121-122 nm measured by SORCE (Rottman et al. 2006) rescaled to Mars distance, with accounting for the different ecliptic longitudes of Earth and Mars as seen from the Sun. The brightness at the center of the line is derived from the empirical relation given by Emerich et al. (2005). The variability of the solar flux between 121 and $122 \mathrm{~nm}$ measured by SORCE during the full period is $\sim 10 \%$, therefore uncertainty on the flux at Mars, due to the different ecliptic longitudes of Mars and Earth should be at most $\sim 10 \%$ but could differ from one observation to the other. Due to the large CPU time required by the Monte Carlo radiative transfer simulations, it is not possible to simulate the Martian Lyman- $\alpha$ volume emission rate with every GCM-LMD time step. In this study, we consider only monthly average hydrogen for the exosphere and corona. This is another source of uncertainty in the simulated brightness that prevents us from discussing differences less than a few tenths percent in the brightness comparisons. This resolution is enough to discuss the seasonal variations that are important, as observed from several missions (Chaffin et al. 2014, 2018, Clarke et al. 2014, Battacharyya et al. 2015, 2017).

\section{1) Thermospheric density}

The GCM-LMD is a 3D model of the Martian atmosphere from the surface to the exobase. It is composed of a 3D dynamics core which solves the fluid equations over a sphere and a physical core, describing all the physical processes computed for each column. In all the simulations presented below, the horizontal resolution is $5.625^{\circ}$ in longitude and $3.75^{\circ}$ in latitude. The dynamical time step $\delta \mathrm{t}$ is $1 \mathrm{sol} / 960 . \sim 1.5$ minute, and the physics time step is $\Delta \mathrm{t}$ $=5 \delta \mathrm{t} \sim 7.5$ minutes. More details on the GCM-LMD can be found in Forget et al. (1999) and Gonzalez-Galindo et al. (2009, 2015). As noted in Chaufray et al. (2015a) the vertical resolution used in the molecular diffusion (2 km) can lead to an uncertainty of a few tenths in the hydrogen density above $200 \mathrm{~km}$, depending on the season and martian year. Because we will focus on large differences between the simulated brightness and observed brightness, this uncertainty as a monthly average exosphere, should not change our conclusion.

In these simulations, we account for the daily variations of the solar flux during the Martian year 28 and 29 as computed by Gonzalez-Galindo et al. (2015). We also consider the dust scenarios of these years derived by 
Montabone et al. (2015). We do not include the microphysical processes controlling the supersaturation presented in Navarro et al. (2014), as these results, and especially the hydrogen density in the Martian thermosphere, depend on model parameters not well constrained by the observations. These simulations will be discussed in section 5 . We also do not include the coupling with the exospheric ballistic transport developed by Chaufray et al. (2018). This coupling can't be performed for a full Martian year because of the large CPU time needed. The first few studies we performed indicate that the effect on the brightness should not be greater than a few tens of percent, and therefore, the differences discussed in section 5 should still be present with this coupling. A study of the local time variations would require such a coupling. Therefore, in this paper we will focus on the seasonal variation of the hydrogen corona and escape. We only consider the hydrogen Jeans escape, computed assuming a Maxwellian distribution at the upper level. Shizgal and Blackmore (1986) have shown that the depletion of energetic atoms could reduce the real escape rate by a factor $\sim 0.57$ for an exospheric temperature of $230 \mathrm{~K}$ and 0.53 for an exospheric temperature of $310 \mathrm{~K}$, We also neglect the non-thermal escape processes which should be not very efficient for atomic hydrogen compared to the thermal escape rate (Krasnopolsky 2010). The non-thermal escape of molecular hydrogen was discussed in Krasnopolsky (2017) and Chaufray et al. (2017) and is not included in these simulations.

The simulated escape flux for the Martian years 28 and 29, as well as the escape flux derived from the monthly average conditions for Martian year 28 are displayed on Fig.2. These variations are close to the simulated escape rates for solar minimum conditions by Chaufray et al. (2015a; 2018) as expected for these Martian years. 


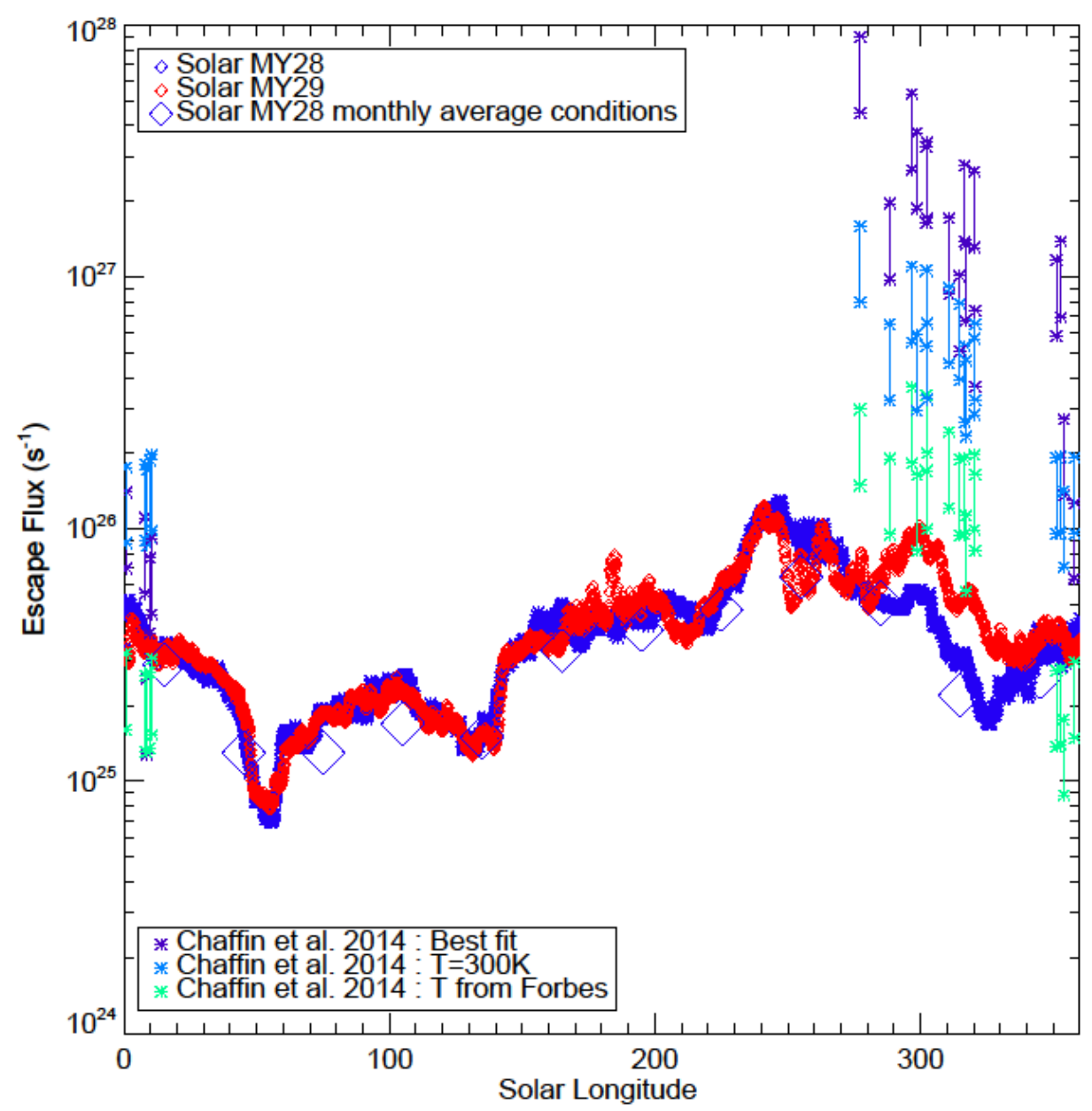

Fig. 3 : Jeans hydrogen escape rate simulated for the Martian years 28 (blue) and 29 (red) with the GCM-LMD. The escape rate derived from the monthly average upper atmosphere are also indicated by the blue diamonds. The escape rates derived from SPICAM fit (from observations between end of Martian year 28, beginning of Martian year 29) using different assumptions by Chaffin et al. 2014 are also displayed. The escape flux $\left(\mathrm{at} / \mathrm{cm}^{2} / \mathrm{s}\right)$ was converted to escape rate (at/s). For this conversion, we assume either a uniform flux at the exobase or a uniform flux at the dayside exosbase and no escape flux at the nightside, leading to the range given by each vertical line.

\section{2) Exospheric density}

The hydrogen density is extended to the exosphere (above $200 \mathrm{~km}$ ), by solving the Liouville equations from nonuniform conditions at the exobase (Vidal-Madjar and Bertaux 1972). We consider Maxwellian velocity distribution functions at the exobase using the temperature and the hydrogen density computed from the LMD-GCM reinterpolated at $200 \mathrm{~km}$ as done in our previous simulations (Chaufray et al. 2015a, 2018). The real distribution at the exobase should differ from a Maxwellian distribution. Due to the escaping particles, the Maxwellian distribution is truncated at velocity larger than the escape velocity (Shizgal and Blackmore 1986, Boqueho et al. 2005, Terada et al., 2016). However, this truncation should not affect the ballistic distribution with a velocity lower than the escaping 
velocity but only reduce the escape rate (Brinkmann, 1970). The Direct Simulation Monte Carlo method used by Terada et al. (2016) shows that the ballistic hydrogen population is very close to the Maxwellian distribution at the local temperature (see Fig 8 b in Terada et al. 2016).

In the computation of the exospheric hydrogen density we neglect ionization loss and the effect of solar radiation pressure that should be important at a few Martian radii above the altitudes scanned by the missions and the optically thick region of the hydrogen corona (Beth et al.2014).

\section{3) Radiative transfer model for resonance line}

The computation of the Lyman- $\alpha$ brightness is done in two steps, as done by numerous models (e.g., Gladstone 1992). In a first step, we use a 3D Monte Carlo radiative transfer model to compute the $3 \mathrm{D}$ volume emission rate from $80 \mathrm{~km}$ to $50,000 \mathrm{~km}$. This radiative transfer model is a 3D version of the model used by Chaufray et al. (2012, 2015b) to study the hydrogen corona of Venus, which accounts for angle-dependent partial frequency redistribution (Lee 1982) and describes the spectral line from -5 to +5 Doppler width (at the maximum temperature at the exobase, i.e., $\sim \pm 40 \mathrm{~mA})$.

The brightness is then computed in a second step, rescaling the volume emission rate to the solar flux at Lyman- $\alpha$ at the time of the observations and accounting for the geometry of each individual observation from Mars Express (Chaufray et al. 2008). Twenty-four 3D volume emission rates, monthly average, corresponding to Martian months of the years 28 and 29 have been computed. To reduce the CPU time, we did not simulate every line of sight for each observation, but instead averaged several lines of sight (between 5 and 20) and compared the simulated brightness of the average line of sight with the observed average brightness.

The interplanetary brightness emission is estimated for each individual observation using a 3D radiative transfer model of the interplanetary emission (Quémerais 2000). The possible uncertainty on the brightness should be small ( 100R) compared to the brightness of the Martian hydrogen corona and will not change the conclusion of this study.

\section{4) Effect of ballistic transport in the Martian exosphere}

The effect of ballistic transport on the hydrogen density near the exobase of Mars has been recently studied (Chaufray et al. 2018). This coupling has not been included in this study due to the large CPU needed to perform such coupled simulations. Our first estimates indicate that considering this coupling has a small effect on the 
simulated brightness. The Lyman- $\alpha$ emission line is optically thick below few thousands kilometers in altitude (Bhattacharyya et al. 2017) and therefore is sensitive to global conditions and not too significantly affected by local conditions. Ballistic transport redistributes the hydrogen atoms in the exosphere, but does not change the global amount of hydrogen in the exosphere. Therefore, it is not surprising to find a limited impact on the simulated brightness. Since the main goal of this study is to focus on the large differences between the simulations and the observations, in the next section we will neglect this effect. A more careful study of the local time variations by few tenths of the brightness as observed by MAVEN (Chaffin et al. 2015) would need to include this effect and such a study is therefore deferred to a future work.

\section{4) Comparisons with observations}

Examples of comparisons between the observed brightness and the simulated brightness at different Martian months are displayed on Fig. 4. The model reasonably reproduces the observations at Ls $=180^{\circ}, 220^{\circ}$ and $340^{\circ}$ but underestimates the brightness at $\mathrm{Ls}=280^{\circ}$ and does not reproduce the shape of the vertical profile. To quantify the difference between the observed and simulated brightness, we derived an optimized scale factor which needed to be applied to the observation to better reproduce the simulated profile by minimizing the function.

$$
\Delta^{2}=\sum_{\text {los }}\left[\log \left(I_{o b s}\right)+\log (A)-\log \left(I_{\text {sim }}\right)\right]^{2}
$$

The minimum of the function $\Delta^{2}$ is found when its derivative $\partial \Delta^{2} / \partial \mathrm{A}=0$ yielding $\mathrm{A}$ as

$$
\log (A)=\frac{1}{n} \sum_{\text {los }}\left[\log \left(I_{\text {sim }}\right)-\log \left(I_{\text {los }}\right)\right]
$$

The rescaled profile is also displayed on Fig.4. For the three observations at $\mathrm{Ls}=180^{\circ}, 220^{\circ}$ and $340^{\circ}$ displayed in Fig. 4 , this scale factor is $0.99,0.79$, and 0.82 respectively, and is in the range of possible uncertainty associated with the retrieved brightness and the solar flux at Mars or the simulation of a monthly average hydrogen corona. For the observation near Ls $=280^{\circ}$, the scale factor is 0.39 , which cannot be explained by the uncertainty in the retrieved brightness by SPICAM-UV.

The difference between the simulated and the observed Lyman- $\alpha$ brightness indicates an important underestimate of the hydrogen density by the model, as was suggested by our first simple comparison of the escape rate derived from SPICAM by Chaffin et al. (2014) at this season (from the same set of observations) and the simulated escape rate for different solar conditions (Chaufray et al. 2015a). The shape of the simulated profile also differs from that of the 
observed profile, confirming that the difference cannot be attributed to the absolute calibration of SPICAM or an

underestimate of the solar flux at Mars and is therefore due to an underestimate of the hydrogen density at this season by our model. Observations performed simultaneously between Ls $=331$ and $345^{\circ}$ by HST during the Martian year 28 also indicate a decrease of the hydrogen corona from this period (Clarke et al. 2014), which is consistent with the SPICAM observations.

Possible explanations of this underestimate of the hydrogen content of the Martian upper atmosphere in our model will be discussed in section 5
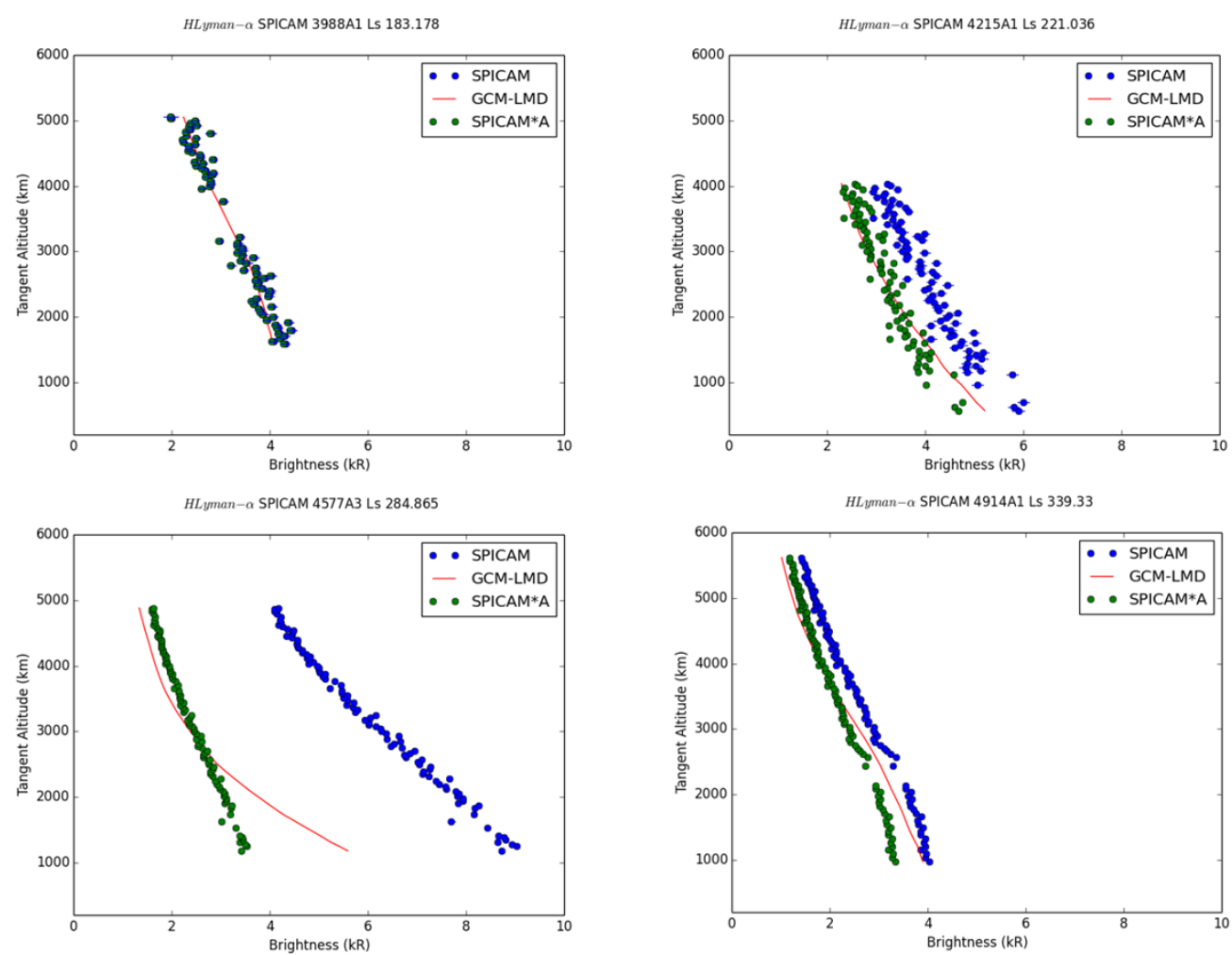

Fig. 4 Examples of comparisons between the Lyman- $\alpha$ brightness profiles observed by SPICAM-UV (blue dots) and the simulated brightness profile (red line) for different solar longitude Ls during the Martian year 28.

Fig. 5 displays the simulated profile corresponding to observation 4577 when the simulated hydrogen density is arbitrarily multiplied by a factor 6 . In that case, the simulated profile is in better agreement with the observation and the simulated shape of the profile is consistent with the observed shape, confirming the difference is likely due to an underestimate of the hydrogen density by the model. 


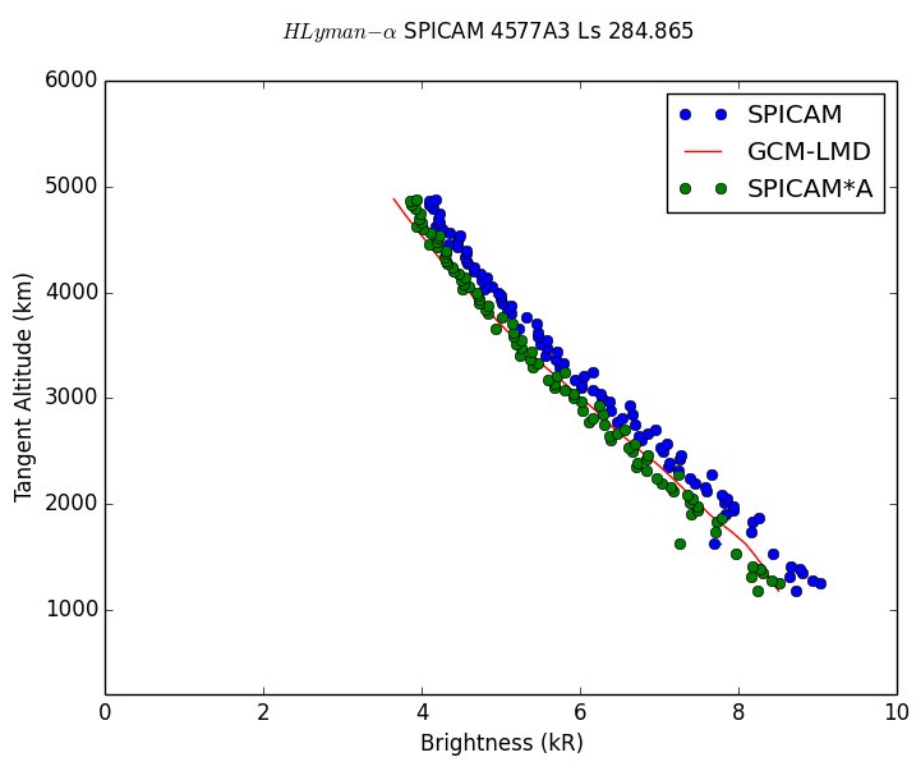

Fig. 5 Simulated Lyman- $\alpha$ brightness profile for orbit \#4577 with a simulated hydrogen density multiplied by 6 w.r.t. the normal model, compared to the SPICAM

Examples of comparisons between the observed brightness and the simulated brightness at different Martian months for Martian year 29 are displayed on Fig. 6. The model reasonably reproduces the observations at Ls near $40^{\circ}, 80^{\circ}$ (the best comparison for the full set of observations), and to a lesser extent, near $145^{\circ}$, but fails to reproduce the observation near $\mathrm{Ls}=255^{\circ}$. The observation at $\mathrm{Ls}=255^{\circ}$ corresponds to a grazing limb observation with a tangent altitude decreasing at the nightside (brightness $<4 \mathrm{kR}$ ) reaching a minimum altitude near $100 \mathrm{~km}$ and increasing on the dayside where the Lyman-alpha brightness is large, $\sim 10 \mathrm{kR}$.

For the Martian year 29, we derive an optimized scale factor $A$ for each observation. The scale factors are $1.08,0.98$, 0.81 and 0.75 for the four profiles displayed on Fig. 5., confirming that the model underestimates the brightness near summer southern solstice. While the factor 0.75 is close to the reasonable value, it corresponds to a large difference in term of hydrogen density, due to the large brightness $(\sim 10 \mathrm{kR})$ and the non-linearity between the density and the brightness for an optically thick emission.

As for the Martian year 28, we performed another simulation with the hydrogen density arbitrarily multiplied by 6 for the observation \#6832. Fig. 7 shows that the agreement is slightly better, as expected, but the brightness is still underestimated. But the remaining difference is less than $1 \mathrm{kR}$ and could be due to the deuterium emission not included in our simulations, this could possibly be important at this season at these low altitudes (Clarke et al. 2017, Chaffin et al. 2018). 

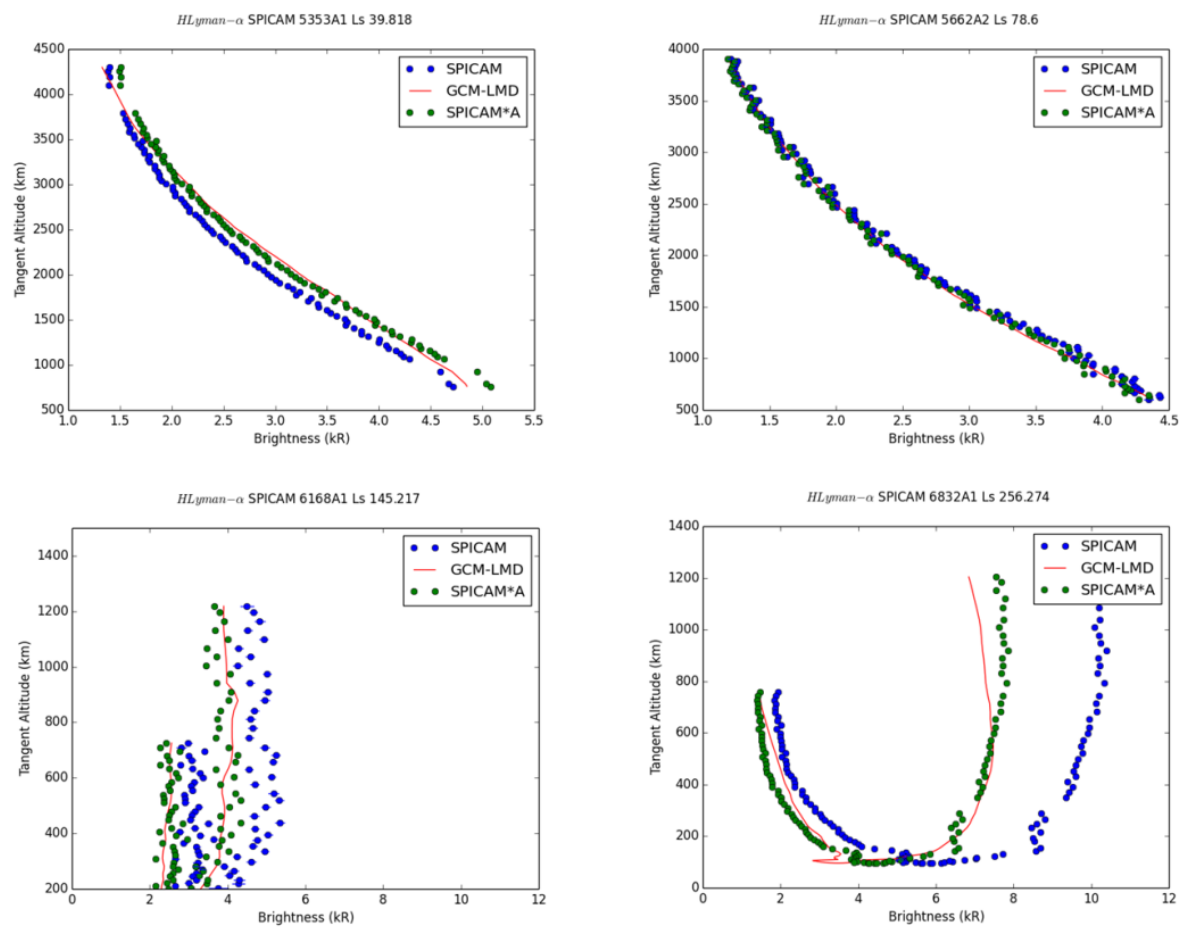

Fig. 6 Examples of comparisons between the Lyman- $\alpha$ brightness profiles observed by SPICAM- UV (blue dots) and the simulated brightness profile (red line) for different solar longitude Ls during the Martian year 29

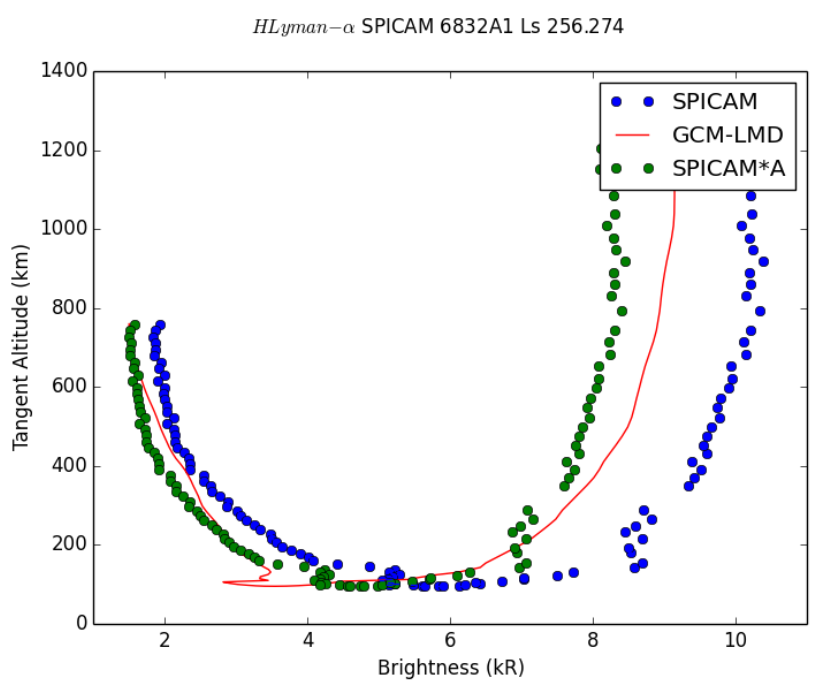

Fig. 7 Simulated Lyman- $\alpha$ brightness profile for orbit \#6832 with a simulated hydrogen density multiplied by 6 w.r.t. the normal model, compared to the SPICAM

For each observation from Martian years 28 and 29, we derive a scale factor and compute the $\chi^{2}$ value for the observed and the rescaled profiles given by:

$$
\chi^{2}=\frac{1}{n} \sum_{l o s} \frac{\left[B^{*} I_{o b s}-I_{s i m}\right]^{2}}{B^{2} \sigma_{o b s}^{2}}
$$

with $\mathrm{B}=1$ for the observed profile and $\mathrm{B}=\mathrm{A}$ for the rescaled profile.

290 Fig.8 displayed the $\chi^{2}$ value versus Ls, including all observations given in Table 1, as well as the scale factor $A$ 

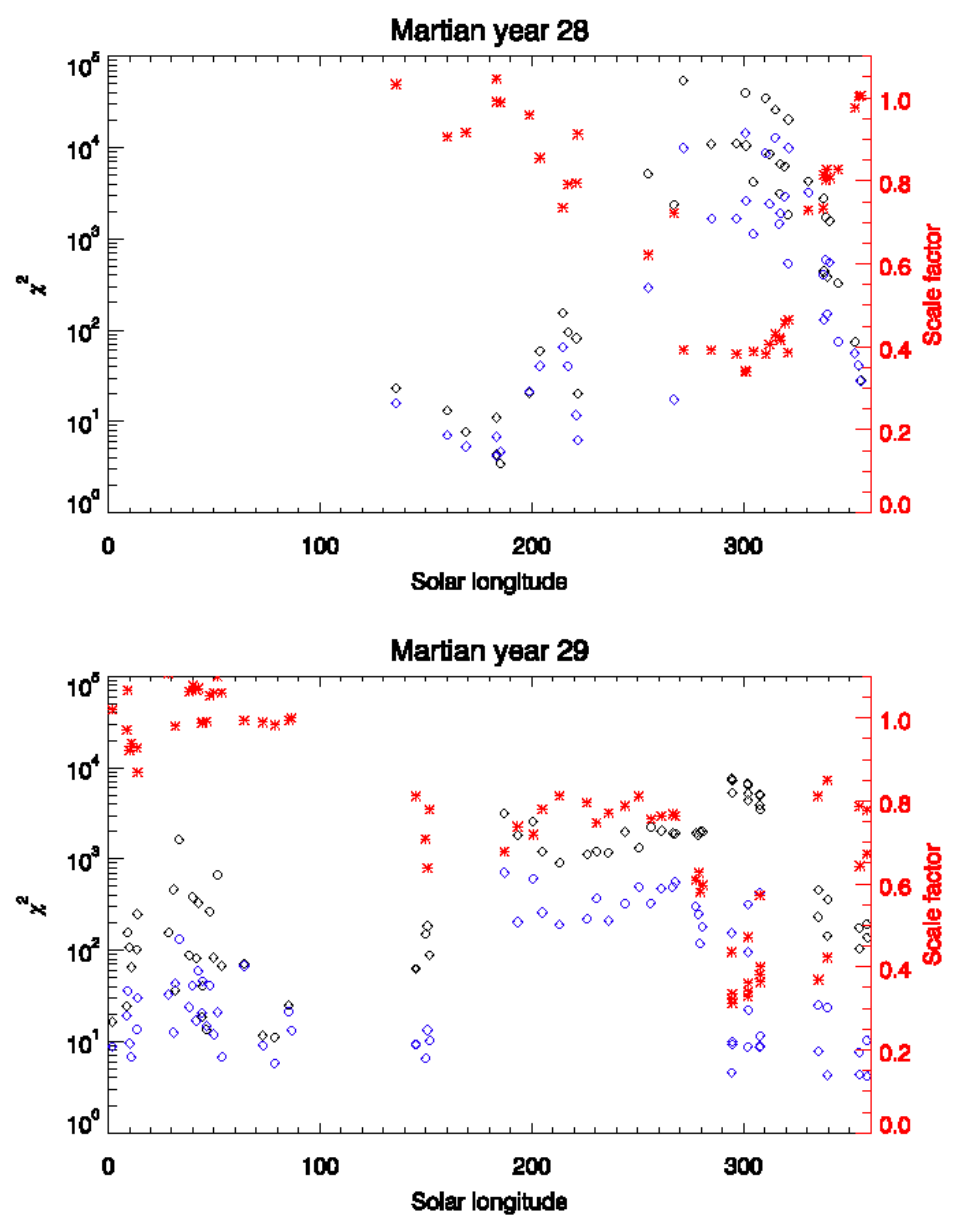

Fig. $8 \chi^{2}$ variations (black diamond, left scale) for the Martian year 28 (top) and Martian year (29), and best scale factor variations (red crosses, right scale), with the associated $\chi^{2}$ values (blue diamonds).

The full set of observations confirm the conclusion derived from the few examples shown in Figs. 4 and 6 . The simulated profiles are in reasonable agreement with the observed profile when $\chi^{2}<100$, and the scale factor is larger than $0.8\left(\mathrm{Ls}<230^{\circ}\right.$ and $\left.\mathrm{Ls}>330^{\circ}\right)$ for Martian year 28, and $\mathrm{Ls}<150^{\circ}$ and $\mathrm{Ls}>340^{\circ}$ for Martian year 29). The scale factor reaches the smallest values $\sim 0.3-0.4$ for $\mathrm{Ls}=250^{\circ}-330^{\circ}$ for both years, meaning the model strongly underestimates the hydrogen density for this season. For Martian year 28, even if the absolute brightness was rescaled, the shape of the profile would not be well reproduced as indicated by the large value of $\chi^{2}$ (see also Fig. 4 bottom left) when the optimized scale factor is included. To reproduce the observed profile, the simulated hydrogen density should be multiplied by $\sim 6$ as shown for orbit 4577 in Fig. 5 .

At Ls near $0^{\circ}$ (observation \#5070), Chaffin et al. (2014) was able to fit the observed profile with an escape flux between $1 \times 10^{7}$ and $1 \times 10^{8} \mathrm{~cm}^{-2} \mathrm{~s}^{-1}$, corresponding to a total escape rate $\sim 1.6 \times 10^{25}$ and $1.6 \times 10^{26} \mathrm{~s}^{-1}$ if we assume a 
uniform escape flux at the exobase in agreement with our simulated escape rate equal to $4 \times 10^{25} \mathrm{~s}^{-1}$ at this season (Fig. 3). The escape flux simulated is not uniform and larger at the dayside than nightside (Chaufray et al. 2015a). If we integrate only the escape flux at the dayside to derive the escape rate, then all the derived escape rate from SPICAM shown in Fig. 3 should be divided by two. In that case, the observations near Ls $=0^{\circ}$ are still in agreement with our simulations. The two other observations detailed in Chaffin et al. (2014) (observation \#4646 and \#4501) are not reproduced by our simulations and the escape rate derived by Chaffin et al. (2014) was between $2 \times 10^{8}$ and $5 \times 10^{9} \mathrm{~cm}^{-}$ ${ }^{2} \mathrm{~s}^{-1}\left(3 \times 10^{26}\right.$ and $\left.8 \times 10^{27} \mathrm{~s}^{-1}\right)$ for observation \#4501 $\left(\mathrm{Ls}=271^{\circ}\right)$ and between $1 \times 10^{8}$ and $2 \times 10^{9} \mathrm{~cm}^{-2} \mathrm{~s}^{-1}\left(1.6 \times 10^{26} \mathrm{~s}^{-1}\right.$ $\left.3.2 \times 10^{27} \mathrm{~s}^{-1}\right)$ for observation $\# 4646\left(\mathrm{Ls}=296^{\circ}\right)$, larger than our simulated escape flux of $\sim 10^{26} \mathrm{~s}^{-1}$ at these periods

(Fig. 2). These high values would be in better agreement with the model escape flux if the simulated hydrogen density was increased by a factor $\sim 6$ as suggested by our simple density rescale.

\section{5) Discussion}

The hydrogen density in the Martian upper atmosphere simulated for Martian years 28 and 29 is in general in reasonable agreement with the SPICAM observations suggesting that the main source processes of atomic hydrogen are included in these simulations during most of the Martian year, except near southern summer solstice $\left(\operatorname{Ls} \sim 270^{\circ}\right)$. At this period, the hydrogen density and the escape rate are likely underestimated by a factor $\sim 6$.

At this period, deuterium Lyman- $\alpha$ could contribute partly to the brightness, especially in the thermosphere / lower exosphere as shown by MAVEN/IUVS (Clarke et al. 2017), for example for orbits 6500s, 6600s and 6700s (Fig. 6 bottom right). However, the deuterium brightness measured by MAVEN/IUVS during Martian year 32 is less than 1 kRayleigh and decreases quickly with altitude, becoming negligible above $300 \mathrm{~km}$. Such a low brightness is insufficient to explain the discrepancy between the model and the observations. Substantial amounts of water vapor at high altitude in the mesosphere has been detected by SPICAM at this season (Maltagliati et al. 2013, Fedorova et al. 2018). The water vapor could be photodissociated and contribute to an increase in the hydrogen density in the Martian thermosphere and exosphere, as well as the hydrogen escape (Chaffin et al. 2017). The MAVEN/NGIMS ionospheric observation above $150 \mathrm{~km}$ near the same season (Martian year 32) suggested a water vapor mixing ratio of only $0.4 \mathrm{ppb}$ at $80 \mathrm{~km}$, in order to avoid the loss of observed $\mathrm{HCO}^{+}$by $\mathrm{H}_{2} \mathrm{O}$ reactions (Fox et al. 2015), but has been recently questionned by another model (Krasnopolsky 2019). In this study, the water vapor density profile was assumed to be close to a diffusion equilibrium profile. Recently, Heavens et al. (2018) suggested that the increase of the hygropause altitude due to dust storms could lead to an increase of the hydrogen density (and escape) in the 
thermosphere, while Shaposhnikov et al. (2019) suggest, using a GCM, that a "pump" mechanism could facilitate upward transport at high latitude during perihelion. Seasonal variations of the altitude of the hygropause at Mars are present in the LMD-GCM simulations (Montmessin et al. 2005). Fig. 9 displays a typical density profile for $\mathrm{H}_{2} \mathrm{H}_{2}$, $\mathrm{H}_{2} \mathrm{O}$, and $\mathrm{CO}_{2}$ and the temperature profile obtained for Martian year 28 at the equator at noon for the month Ls = $120-150$ and $\mathrm{Ls}=270^{\circ}-300^{\circ}$. At Ls $=120-150^{\circ}$, the water vapor is confined to very low altitudes $(<20 \mathrm{~km})$. The hydrogen density presents a double-peaked profile, one peak in the low atmosphere due to water vapor photodissociation, and another peak in the low thermosphere due to ionospheric reactions (Krasnopolsky 2002). At Ls $=240-270^{\circ}$, the hygropause is $\sim 50 \mathrm{~km}$, but there are still substantial amounts of water vapor in the mesosphere near $80 \mathrm{~km}$ but not above $120 \mathrm{~km}$. Only one hydrogen density peak is observed, because the peak associated with ionospheric reactions is merged with the hydrogen coming from water photodissociation transported from the lower atmosphere which is $\sim 100$ times larger.
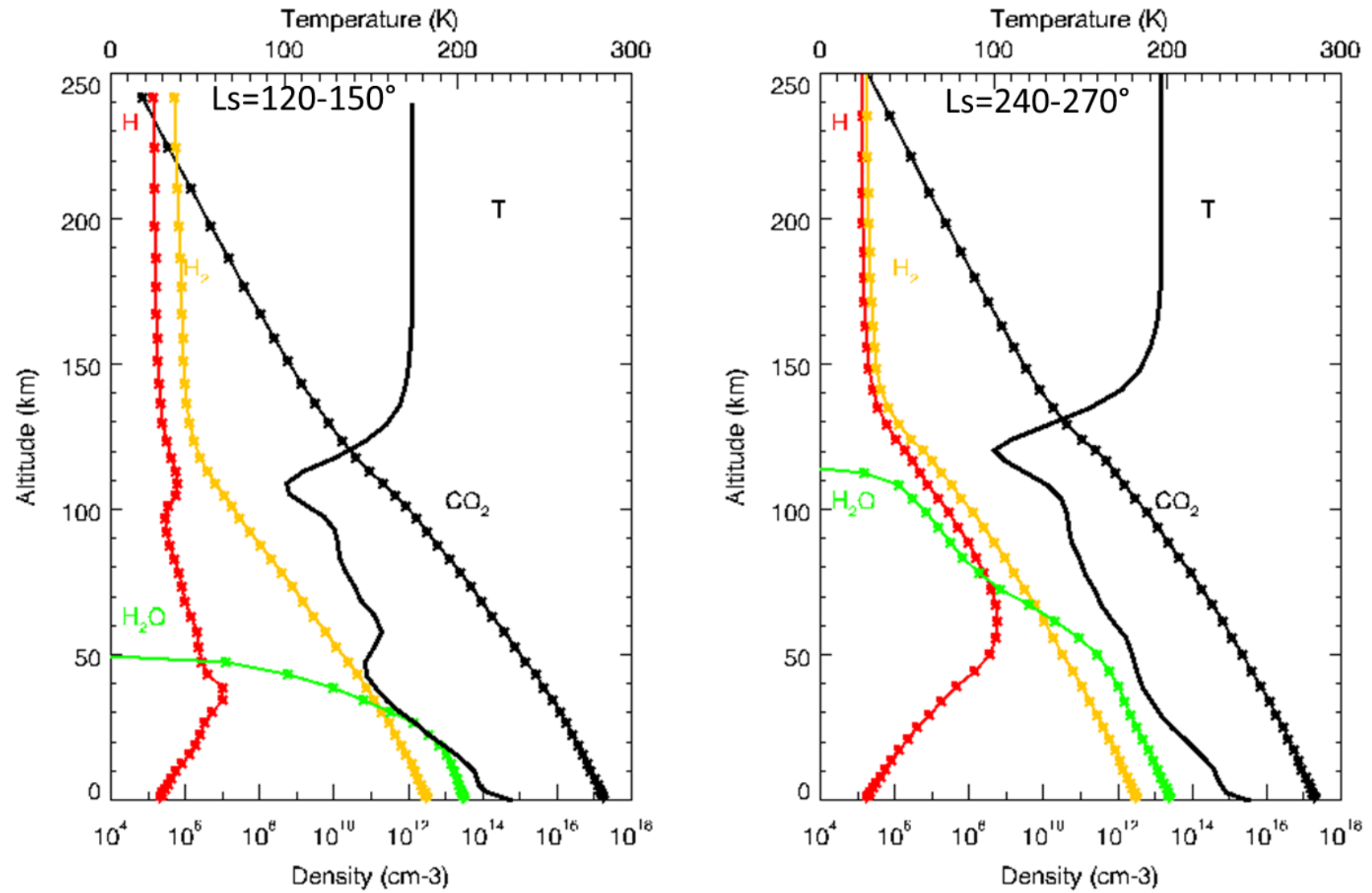

Fig. 9 Vertical density profile of the main hydrogen species and $\mathrm{CO}_{2}$ at noon equator from the surface to the exobase simulated for two different months during the Martian year 28.

The dissociation of water vapor is a source of atomic hydrogen in the Martian lower thermosphere. Such an effect 
could be the main driver of our simulated escape rate, contrary to our first interpretation (Chaufray et al. 2015a). This would partly explain the difference between our simulations and the model of Krasnopolsky (2002), as suggested by Krasnopolsky (2017). An increase in the water vapor density could explain the current underestimate of the hydrogen escape at Ls $=270^{\circ}-300^{\circ}$, and would be in agreement with the conclusion of Maltagliati et al. (2013), who found that the GCM-LMD underestimates the water vapor in the Martian mesosphere at this season, since the description of the water cycle presented in this study is done with similar assumptions than those used by these authors. This discrepancy was attributed to an underestimation of the strength of the interaction between the water vapor and the dust cycles. The water vapor mixing ratio in the mesosphere depends on the supersaturation of the upper atmosphere, which is not well known. The microphysical processes controlling supersaturation are now included in the LMDGCM (Navarro et al. 2014), but their effects on the water vapor at high altitudes depends on model parameters not well constrained by the observations and therefore were not included in these simulations. A similar comparison with the few SPICAM observations displayed in Fig. 4 of the simulations used to generate the Mars Climate Database 5.2 (for the Martian year 28), including these microphysical processes is displayed in Fig. 10
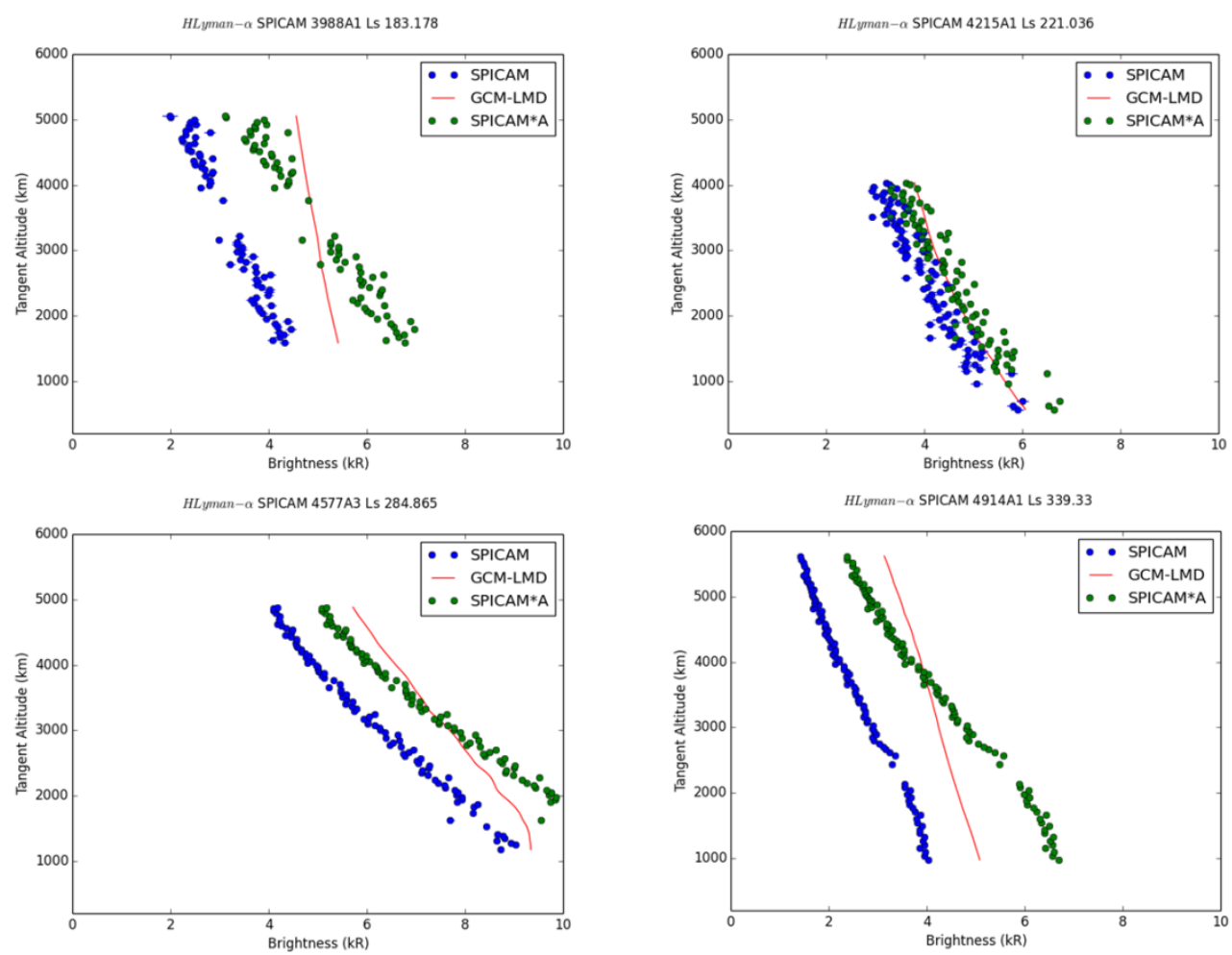

Fig. 10 Same as Fig. 4 but for the simulations used to generate the MCD 5.2, including the microphysical processes controlling the supersaturation.

In these simulations, the Lyman-alpha brightness is overestimated, even at the southern summer. The brightness 
profiles for observations $4215 \mathrm{~A} 1$ is in good agreement, while the simulated shape profiles of the three other observations is very different from the observed shape suggesting that the difference is due to an overestimate of the hydrogen density in the Martian upper altitude. The average hydrogen escape rate for Ls $=270-300^{\circ}$ used to simulate the observation 4501 is $1.1 \times 10^{27} \mathrm{~s}^{-1}$, larger by $\sim 2$ compared with the escape rate estimated in section 4 after a global rescale of the hydrogen density. Although not perfect, the updated LMD-GCM microphysical processes could be sufficient to produce the large amount of hydrogen lacking in our previous simulations. Our simulations could also be consistent with the ionospheric observations by MAVEN/NGIMS, because if the amount of water vapor coming from the lower atmosphere is larger at $120 \mathrm{~km}$, but remains negligible above $150 \mathrm{~km}$, it could prevent the $\mathrm{HCO}^{+}$ destruction while producing $\mathrm{H}_{3} \mathrm{O}^{+}$ions near $120 \mathrm{~km}$ (Fox et al. 2015). A more detailed study of the link between the water vapor and the atomic hydrogen and ionospheric water species will benefit from future observations of the water vapor density in the Martian mesosphere/thermosphere by Exo Mars Trace Gas Orbiter (Lopez-Valverde et al. 2018), especially during the recent global dust storm in June 2018. Comparisons of other Martian years like Martian year 32 where an increase of water vapor was also observed by SPICAM (Fedorova et al. 2018), and using the large Lyman- $\alpha$ dataset of MAVEN/IUVS (Chaffin et al. 2015, 2018) will be also useful to estimate the year to year variability possibly due to dust events and the variability associated with the solar activity in order to extrapolate past conditions with larger EUV solar flux and orbit parameters. Space weather events also increase the atmospheric escape rates (Jakosky et al.. 2015). But they mostly increase the heavy ions escape rates as reported in Jakosky et al. (2015) during the interplanetary coronal mass ejection in March 2015. Solar flares could heat the Martian upper atmosphere and increase the escape rate during short time periods (Mayyasi et al. 2018).

Considering the discrepancy of our model and the SPICAM observations, we estimated the hydrogen escape rate during these two Martian years to vary between $\sim 10^{25}$ to $6 \times 10^{26} \mathrm{~s}^{-1}$. This range is in good agreement the with the seasonal variations $\left(3 \times 10^{25} \mathrm{~s}^{-1}\right.$ near aphelion and $4 \times 10^{26} \mathrm{~s}^{-1}$ near perihelion) reported from pick-up protons for the Martian years 32-33 by Rahmati et al. (2018), and from Lyman- $\alpha$ emission by Chaffin et al. (2018) (estimated $~ 5$ $14 \times 10^{26} \mathrm{~s}^{-1}$ at Ls $=250^{\circ}$ for MY 32 and $1-4 \times 10^{26} \mathrm{~s}^{-1}$ at Ls $=200^{\circ}$ for MY 33). It is slightly lower than the escape rate estimated from the hydrogen column upstream of the bow shock by Halekas (2017); considering $\mathrm{T}=200 \mathrm{~K}$ near aphelion, and $\mathrm{T}=300 \mathrm{~K}$ near perihelion, and assuming an uniform escape rate, the escape flux derived from Fig. 5 of Halekas (2017) leads to an escape rate variation from $\sim 3 \times 10^{25}$ to $\sim 3 \times 10^{27} \mathrm{~s}^{-1}$. If we express this escape flux into a water Global Equivalent Layer (GEL) $d$ using 
$d(m / G y r)=\frac{\Phi_{H}}{2} \frac{m_{H_{2} O}}{\rho_{H_{2} O} S} T$,

where $\Phi_{H} / 2$ is the water loss rates, $\mathrm{m}_{\mathrm{H} 2 \mathrm{O}}$ the mass of a water molecule, $\rho_{\mathrm{H} 2 \mathrm{O}}$ water mass density, $S$ the Martian surface and $T$ the number of seconds in $1 \mathrm{Gyr}\left(\sim 3.2 \times 10^{16} \mathrm{~s} / \mathrm{Gyr}\right)$, we find a loss between $\sim 33$ to $2000 \mathrm{~mm}$ per billion years and therefore no more than $10 \mathrm{~m} \mathrm{GEL}$ during the last 4 billion years, which is small compared to the total water content measured in the reservoirs at the surface and the subsurface $(\sim 30 \mathrm{~m})$, and even less compared to the estimated amount of water needed to form the valley networks or outflow channels (estimated to few 100s m GEL with large uncertainties, Lasue et al. 2013). The differences could be either due to a more important hydrogen escape rate in the past or to a larger reservoir of water (ice or liquid) in the subsurface inaccessible or not yet detected by current radar (Orosei et al. 2015, 2018).

\section{6) Conclusion}

We simulated the atomic hydrogen content in the upper atmosphere of Mars for Martian years 28 and 29. These two years correspond to a period of numerous hydrogen coronal observations by SPICAM on Mars Express. The comparison between our simulated brightness and SPICAM observations shows a reasonable agreement with the observations for $\mathrm{Ls}<200^{\circ}$ and $\mathrm{Ls}>330^{\circ}$ for Martian year 28, for $\mathrm{Ls}<150^{\circ}$ and Ls $>340^{\circ}$ for Martian year 29, while the simulated brightness is strongly underestimated for $230<\mathrm{Ls}<330^{\circ}$ for Martian year 28 and $270^{\circ}<\mathrm{Ls}<340^{\circ}$ for Martian year 29 (therefore, around and after the southern solstice equinox at $\mathrm{Ls}=270^{\circ}$ and the perihelion crossing at $\mathrm{Ls}=251^{\circ}$ ). This underestimate corresponds to a model underestimate of the global hydrogen density (and the Jeans escape rate) by a factor $\sim 6$. Therefore, our study confirms that the seasonal variations of the hydrogen escape at Mars could be of almost two orders of magnitude, as suggested by Chaffin et al. (2014). It also confirms that this variation is a seasonal variation which may be expected every Martian year, as observed from plasma measurements by Mars Express (Yamauchi et al. 2015) and more recently by MAVEN for the Martian year 32, and 33 (e.g., Clarke et al. 2017, Halekas 2017, Rahmati et al. 2018, Chaffin et al. 2018). The discrepancy between our simulations and the observations is attributed to an LMD-GCM underestimate of the amount of water vapor transported to high altitudes. Accurate observations of the water vapor density in the Martian upper atmosphere by TGO_Vandaele et al. 2019) or MAVEN/NGIMS could help to better the understanding of the details of this transport.

\section{Acknowledgements}


424

This project was partially funded by the Programme National de Planetologie and Programme National Soleil Terre and by the Centre National d'Etudes Spatiales. This work has been partially funded by the European Union Horizon 2020 Programme (H2020 Compet -08-2014) under grant agreement UPWARDS-633127. We would like to thank G. Lacombe and L. Baggio for their support on the calibration. We thank Allyson Leffler for her careful review of grammatical aspects of this paper. The SPICAM/MEX data used in this study are available through the ESA Planetary Science Archive (PSA, https://archives.esac.esa.int/psa/). The monthly average 3D simulated hydrogen exosphere can be found on : https://owncloud.latmos.ipsl.fr/index.php/s/AdUgv9PdipA2rim

\section{Appendix : List of SPICAM-UV observations used in this study.}

The list of the SPICAM observations used for the Martian year 28 and 29, and some geometric parameters are given in Table 1 and Table 2 respectively

\begin{tabular}{|c|c|c|c|c|c|c|c|c|}
\hline $\begin{array}{l}\text { Orbit and } \\
\text { Sequence }\end{array}$ & Date & Ls & $\begin{array}{l}\text { Altitude } \\
\text { range }(\mathrm{km})\end{array}$ & $\begin{array}{l}\text { SZA } \\
\text { range }\end{array}$ & $\begin{array}{l}\text { Latitude } \\
\text { range }\end{array}$ & $\begin{array}{l}\text { Slit/N } \\
\text { o slit }\end{array}$ & Binning/ $Y_{0}$ & $\begin{array}{l}\text { Band } \\
\text { used }\end{array}$ \\
\hline 3668A1 & 15 Nov 2006 & 135.4 & $600-5600$ & $56^{\circ}$ & $32 \mathrm{~S}$ & Slit & $32 / 22$ & 5 \\
\hline $3838 \mathrm{~A} 2$ & 02 Jan 2007 & 159.8 & $500-5500$ & $57^{\circ}$ & $7 \mathrm{~N}$ & Slit & $32 / 22$ & 5 \\
\hline 3896A1 & 18 Jan 2007 & 168.4 & $600-5600$ & $66^{\circ}$ & $5 \mathrm{~N}$ & Slit & $32 / 22$ & 5 \\
\hline 3988A1 & $13 \mathrm{Feb} 2007$ & 182.9 & $600-5000$ & $79^{\circ}$ & $23 \mathrm{~S}$ & Slit & $32 / 22$ & 5 \\
\hline 3989A1 & 13 Feb 2007 & 182.9 & $500-4500$ & $79^{\circ}$ & $37 \mathrm{~S}$ & Slit & $32 / 22$ & 5 \\
\hline 4001A1 & 17 Feb 2007 & 185.1 & $500-4800$ & $80^{\circ}$ & $26 \mathrm{~S}$ & Slit & $32 / 22$ & 5 \\
\hline $4085 \mathrm{~A} 1$ & 12 Mar 2007 & 198.6 & $1000-4300$ & $39-67$ & $1 \mathrm{~S}-18 \mathrm{~S}$ & Slit & $32 / 22$ & 5 \\
\hline $4114 \mathrm{~A} 1$ & 20 Mar 2007 & 203.3 & $900-4600$ & $40-73$ & $12 S-32 S$ & Slit & $32 / 22$ & 5 \\
\hline 4178A1 & 07 Apr 2007 & 214.3 & $800-4500$ & $50-86$ & $3 \mathrm{~S}-28 \mathrm{~S}$ & Slit & $32 / 22$ & 5 \\
\hline $4192 \mathrm{~A} 1$ & 11 Apr 2007 & 216.7 & $500-4100$ & $50-88$ & $4 \mathrm{~N}-24 \mathrm{~S}$ & Slit & $32 / 22$ & 5 \\
\hline $4215 \mathrm{~A} 1$ & 17 Apr 2007 & 220.4 & $500-4100$ & $55-93$ & $7 \mathrm{~N}-22 \mathrm{~S}$ & Slit & $32 / 22$ & 5 \\
\hline 4219A1 & 18 Apr 2007 & 221.0 & $400-3600$ & $57-92$ & $19 N-13 S$ & Slit & $32 / 22$ & 5 \\
\hline 4407A4 & 10 Jun 2007 & 254.4 & $500-4000$ & $10-80$ & $14 \mathrm{~S}-54 \mathrm{~N}$ & Slit & $32 / 118$ & 4 \\
\hline 4475A1 & 29 Jun 2007 & 266.5 & $400-4200$ & $36-86$ & $4 \mathrm{~S}-58 \mathrm{~N}$ & Slit & $32 / 118$ & 4 \\
\hline 4501A1 & 07 Jul 2007 & 271.5 & $900-4300$ & $14-67$ & $14 \mathrm{~S}-44 \mathrm{~N}$ & No Slit & $32 / 118$ & 1 \\
\hline
\end{tabular}




\begin{tabular}{|c|c|c|c|c|c|c|c|c|}
\hline $4577 \mathrm{~A} 3$ & $28 \mathrm{Jul} 2007$ & 284.6 & $1100-4800$ & $8-60$ & $16 \mathrm{~S}-34 \mathrm{~N}$ & Slit & $32 / 118$ & 4 \\
\hline 4646A1 & 16 Aug 2007 & 296.2 & $1300-5600$ & 58 & $28 \mathrm{~N}$ & Slit & $32 / 118$ & 4 \\
\hline $4671 \mathrm{~A} 2$ & 23 Aug 2007 & 300.4 & $1100-5100$ & $47^{\circ}$ & $10 \mathrm{~N}$ & No Slit & $32 / 118$ & 1 \\
\hline $4673 \mathrm{~A} 2$ & 24 Aug 2007 & 301.0 & $1300-5500$ & $51^{\circ}$ & $17 \mathrm{~N}$ & Slit & $32 / 118$ & 4 \\
\hline $4693 \mathrm{~A} 2$ & 29 Aug 2007 & 304.0 & $300-10000$ & $36-96$ & $5 \mathrm{~N}-76 \mathrm{~N}$ & Slit & $32 / 118$ & 4 \\
\hline 4729A1 & 08 Sep 2007 & 309.8 & $1100-3500$ & $45^{\circ}$ & $23 \mathrm{~S}$ & No slit & $32 / 118$ & 1 \\
\hline 4740A1 & 11 Sep 2007 & 311.6 & $1100-3600$ & $47^{\circ}$ & $25 \mathrm{~S}$ & Slit & $32 / 118$ & 4 \\
\hline $4757 \mathrm{~A} 2$ & 16 Sep 2007 & 314.5 & $700-3600$ & $50^{\circ}$ & $28 \mathrm{~S}$ & No slit & $32 / 118$ & 1 \\
\hline 4769A2 & 20 Sep 2007 & 316.8 & $300-10000$ & $19-85$ & $1 S-63 N$ & Slit & $32 / 118$ & 4 \\
\hline $4772 \mathrm{~A} 2$ & $20 \operatorname{Sep} 2007$ & 316.8 & $700-3600$ & 53 & $31 \mathrm{~S}$ & Slit & $32 / 118$ & 4 \\
\hline $4785 \mathrm{~A} 1$ & 24 Sep 2007 & 319.1 & $700-3600$ & 56 & $33 \mathrm{~S}$ & Slit & $32 / 118$ & 4 \\
\hline 4794A2 & 27 Sep 2007 & 320.8 & $800-10000$ & $19-90$ & $2 \mathrm{~N}-60 \mathrm{~N}$ & Slit & $32 / 118$ & 4 \\
\hline 4796A1 & 27 Sep 2007 & 320.8 & $700-3600$ & 57 & $34 \mathrm{~S}$ & No Slit & $32 / 118$ & 1 \\
\hline 4856A1 & 14 Oct 2007 & 330.2 & $700-3600$ & 69 & $45 \mathrm{~S}$ & No Slit & $32 / 118$ & 1 \\
\hline 4903A1 & 27 Oct 2007 & 337.3 & $1400-5500$ & 76 & $23 \mathrm{~S}$ & No Slit & $32 / 118$ & 1 \\
\hline 4905A1 & 28 Oct 2007 & 337.8 & $700-5600$ & 77 & $10 \mathrm{~S}$ & Slit & $32 / 118$ & 4 \\
\hline 4910A1 & 29 Oct 2007 & 338.4 & $1000-5600$ & 77 & $17 \mathrm{~S}$ & No Slit & $32 / 118$ & 1 \\
\hline 4914A1 & 30 Oct 2007 & 338.9 & $1000-5600$ & 78 & $18 \mathrm{~S}$ & Slit & $32 / 118$ & 4 \\
\hline 4923A1 & 02 Nov 2007 & 340.5 & $700-5000$ & 79 & $34 \mathrm{~S}$ & No Slit & $32 / 118$ & 1 \\
\hline 4950A1 & 09 Nov 2007 & 344.2 & $700-5000$ & 84 & $39 \mathrm{~S}$ & Slit & $32 / 118$ & 4 \\
\hline $5005 \mathrm{~A} 1 \mathrm{a}$ & 25 Nov 2007 & 352.5 & $300-3300$ & $32-56$ & $24 \mathrm{~S}-9 \mathrm{~N}$ & Slit & $32 / 118$ & 4 \\
\hline $5016 \mathrm{~A} 1$ & 28 Nov 2007 & 354.0 & $600-3600$ & $35-59$ & $14 \mathrm{~S}-14 \mathrm{~N}$ & Slit & $32 / 118$ & 4 \\
\hline $5024 \mathrm{~A} 1$ & 30 Nov 2007 & 355.1 & $500-3700$ & $34-61$ & $17 \mathrm{~N}-29 \mathrm{~N}$ & Slit & $32 / 118$ & 4 \\
\hline $5052 \mathrm{~A} 1$ & 08 Dec 2007 & 359.1 & $500-3900$ & $36-66$ & $18 \mathrm{~N}-31 \mathrm{~N}$ & Slit & $32 / 118$ & 4 \\
\hline
\end{tabular}

Table 1 : List of SPICAM-UV observations used to study the Martian hydrogen corona during the Martian year 28. 439 The orbit number and sequence number of the observations are given in column 1. Column 3 gives the approximate solar longitude Ls at the observation day. Columns 4 and 5 refer to the tangent point of the line of sight.

\begin{tabular}{|c|c|c|c|c|c|c|c|c|}
\hline $\begin{array}{l}\text { Orbit and } \\
\text { Sequence }\end{array}$ & Date & Ls & Altitude range & SZA range & Latitude range & $\begin{array}{l}\text { Slit/No } \\
\text { slit }\end{array}$ & $\begin{array}{l}\text { Binning/Y } \\
0\end{array}$ & $\begin{array}{l}\text { Band } \\
\text { used }\end{array}$ \\
\hline 5070A1 & 13 Dec 2007 & 1.6 & $800-4300$ & $41-70$ & $15 \mathrm{~N}-29 \mathrm{~N}$ & Slit & $32 / 118$ & 4 \\
\hline 5097A1 & 21 Dec 2007 & 5.5 & $900-10,000$ & $75-107$ & $0-60 \mathrm{~N}$ & Slit & $32 / 118$ & 4 \\
\hline 5119A1 & 27 Dec 2007 & 8.5 & $1000-4800$ & $50-80$ & $16 \mathrm{~N}-32 \mathrm{~N}$ & Slit & $32 / 118$ & 4 \\
\hline
\end{tabular}




\begin{tabular}{|c|c|c|c|c|c|c|c|c|}
\hline 5122A1 & 28 Dec 2007 & 8.9 & $1000-4500$ & $50-80$ & $7 \mathrm{~N}-25 \mathrm{~N}$ & No Slit & $32 / 118$ & 1 \\
\hline 5129A1 & 30 Dec 2007 & 9.9 & $900-4400$ & $50-80$ & $4 N-22 N$ & Slit & $32 / 118$ & 4 \\
\hline 5135A1 & 31 Dec 2007 & 10.4 & $900-4600$ & $50-82$ & $14 \mathrm{~N}-33 \mathrm{~N}$ & Slit & $32 / 118$ & 4 \\
\hline 5154A1 & 06 Jan 2008 & 13.3 & $950-4500$ & $55-85$ & $0-20 \mathrm{~N}$ & Slit & $32 / 118$ & 4 \\
\hline 5156A1 & 06 Jan 2008 & 13.3 & $1100-4900$ & $56-87$ & $13 \mathrm{~N}-32 \mathrm{~N}$ & Slit & $32 / 118$ & 4 \\
\hline 5266A1 & 07 Feb 2008 & 28.3 & $450-4300$ & $40-99$ & $23 \mathrm{~S}-85 \mathrm{~S}$ & Slit & $32 / 118$ & 4 \\
\hline 5283A1 & 12 Feb 2008 & 30.6 & $700-4500$ & $48-99$ & $34 \mathrm{~S}-71 \mathrm{~S}$ & No Slit & $32 / 118$ & 1 \\
\hline 5290A2 & $14 \mathrm{Feb} 2008$ & 31.5 & $700-4500$ & $48-99$ & $34 \mathrm{~S}-72 \mathrm{~S}$ & Slit & $32 / 118$ & 4 \\
\hline 5304A1 & 18 Feb 2008 & 33.3 & $600-4400$ & $40-97$ & $22 S-83 S$ & No Slit & $32 / 118$ & 1 \\
\hline 5341A1 & $28 \mathrm{Feb} 2008$ & 37.9 & $600-4400$ & $35-95$ & $19 \mathrm{~S}-78 \mathrm{~S}$ & Slit & $32 / 118$ & 4 \\
\hline $25353 \mathrm{~A} 1$ & 03 Mar 2008 & 39.7 & $500-4300$ & $31-92$ & $14 \mathrm{~S}-76 \mathrm{~S}$ & No Slit & $32 / 118$ & 1 \\
\hline 5367A1 & 07 Mar 2008 & 41.5 & $500-4400$ & $33-93$ & $16 \mathrm{~S}-75 \mathrm{~S}$ & Slit & $32 / 118$ & 4 \\
\hline 2 5374A1 & 09 Mar 2008 & 42.4 & $500-4300$ & $30-92$ & $12 \mathrm{~S}-73 \mathrm{~S}$ & No Slit & $32 / 118$ & 1 \\
\hline 5388A1 & 13 Mar 2008 & 44.2 & $600-4000$ & $52-101$ & $29 \mathrm{~S}-59 \mathrm{~S}$ & Slit & $32 / 118$ & 4 \\
\hline 5390A1 & 13 Mar 2008 & 44.2 & $600-4000$ & $52-101$ & $28 \mathrm{~S}-59 \mathrm{~S}$ & No Slit & $32 / 118$ & 1 \\
\hline 5404A1 & 17 Mar 2008 & 45.9 & $600-4000$ & $50-100$ & $26 \mathrm{~S}-62 \mathrm{~S}$ & Slit & $32 / 118$ & 4 \\
\hline 5416A1 & 21 Mar 2008 & 47.7 & $600-4100$ & $47-98$ & $22 \mathrm{~S}-64 \mathrm{~S}$ & No Slit & $32 / 118$ & 1 \\
\hline 5432A1 & 25 Mar 2008 & 49.5 & $600-4100$ & $46-98$ & $21 \mathrm{~S}-65 \mathrm{~S}$ & Slit & $32 / 118$ & 4 \\
\hline 5446A1 & 29 Mar 2008 & 51.3 & $600-4200$ & $43-95$ & $17 S-66 S$ & No Slit & $32 / 118$ & 1 \\
\hline 5462A1 & 03 Apr 2008 & 53.5 & $600-4000$ & $51-100$ & $21 \mathrm{~S}-63 \mathrm{~S}$ & Slit & $32 / 118$ & 4 \\
\hline 5546A1 & 27 Apr 2008 & 64.0 & $400-3600$ & $57-100$ & $12 \mathrm{~S}-59 \mathrm{~S}$ & Slit & $32 / 118$ & 4 \\
\hline $5617 \mathrm{~A} 2$ & 17 May 2008 & 72.7 & $500-4000$ & $40-77$ & $14 \mathrm{~N}-43 \mathrm{~S}$ & Slit & $32 / 118$ & 4 \\
\hline $5662 \mathrm{~A} 2$ & 30 May 2008 & 78.4 & $400-3900$ & $35-70$ & $17 \mathrm{~N}-39 \mathrm{~S}$ & Slit & $32 / 118$ & 4 \\
\hline $5715 \mathrm{~A} 2$ & 14 Jun 2008 & 85.0 & $800-3400$ & $60-91$ & $12 S-46 S$ & No Slit & $32 / 118$ & 1 \\
\hline 5726A2 & 17 Jun 2008 & 86.3 & $900-3300$ & $58-89$ & $11 \mathrm{~S}-45 \mathrm{~S}$ & No Slit & $32 / 118$ & 1 \\
\hline $5759 \mathrm{~A} 2$ & 26 Jun 2008 & 90.7 & $300-1900$ & $30-70$ & $23 \mathrm{~N}-31 \mathrm{~S}$ & Slit & $32 / 118$ & 4 \\
\hline 6168A1* & 21 Oct 2008 & 144.9 & $730-200-1200$ & $105-48-49$ & $52 \mathrm{~S}-61 \mathrm{~N}$ & Slit & $32 / 23$ & 5 \\
\hline $6200 \mathrm{~A} 1 *$ & 30 Oct 2008 & 149.4 & $900-200-1300$ & $101-51-55$ & $64 \mathrm{~S}-61 \mathrm{~N}$ & Slit & $32 / 23$ & 5 \\
\hline $6206 \mathrm{~A} 1 *$ & 01 Nov 2008 & 150.5 & $1200-200-1300$ & $95-59-64$ & $73 \mathrm{~S}-60 \mathrm{~N}$ & Slit & $32 / 23$ & 5 \\
\hline $6213 \mathrm{~A} 1 *$ & 03 Nov 2008 & 151.5 & $900-200-1300$ & $100-50-55$ & $66 \mathrm{~S}-60 \mathrm{~N}$ & Slit & $32 / 23$ & 5 \\
\hline 6440A1 & 07 Jan 2009 & 187.0 & $900-110-1200$ & $87-72-78$ & $73 \mathrm{~S}-23 \mathrm{~N}$ & Slit & $32 / 118$ & 4 \\
\hline 6478A1* & 18 Jan 2009 & 193.3 & $1200-200-600$ & $100-46-49$ & $23 \mathrm{~S}-61 \mathrm{~S}-16 \mathrm{~S}$ & Slit & $32 / 118$ & 4 \\
\hline
\end{tabular}




\begin{tabular}{|c|c|c|c|c|c|c|c|c|}
\hline $6521 \mathrm{~A} 1$ & 30 Jan 2009 & 200.6 & $1300-100-1200$ & $130-35$ & $40 \mathrm{~S}-73 \mathrm{~S}-2 \mathrm{~S}$ & Slit & $32 / 118$ & 4 \\
\hline 6546A1* & 06 Feb 2009 & 204.9 & $1300-200-1100$ & $120-28$ & 27S-65S-13S & Slit & $32 / 118$ & 4 \\
\hline 6593A1* & 20 Feb 2009 & 213.1 & $1100-200-500$ & $120-44$ & $3 \mathrm{~S}-72 \mathrm{~S}-42 \mathrm{~S}$ & Slit & $32 / 118$ & 4 \\
\hline 6666A1 & 13 Mar 2009 & 226.1 & $1300-100-1300$ & $140-12$ & $19 \mathrm{~S}-74 \mathrm{~S}-23 \mathrm{~S}$ & Slit & $32 / 118$ & 4 \\
\hline 6691A3* & 20 Mar 2009 & 230.4 & $1300-200-1300$ & $140-13$ & $14 \mathrm{~S}-66 \mathrm{~S}-26 \mathrm{~S}$ & Slit & $32 / 118$ & 4 \\
\hline $6722 \mathrm{~A} 1 *$ & 29 Mar 2009 & 236.2 & $1300-200-1300$ & $150-20$ & 9S-67S-31S & Slit & $32 / 118$ & 4 \\
\hline 6765A1 & 10 Apr 2009 & 244.1 & $900-100-1200$ & $135-10$ & $18 \mathrm{~S}-70 \mathrm{~S}-24 \mathrm{~S}$ & Slit & $32 / 118$ & 4 \\
\hline 6801A1 & 20 Apr 2009 & 250.0 & $1300-100-1200$ & $140-35$ & $7 \mathrm{~N}-77 \mathrm{~S}-27 \mathrm{~S}$ & Slit & $32 / 118$ & 4 \\
\hline 6832A1 & 29 Apr 2009 & 256.3 & $800-100-1200$ & $140-10$ & 9S-66S-32S & Slit & $32 / 118$ & 4 \\
\hline 6859A1 & 07 May 2009 & 261.2 & $900-90-1200$ & $140-15$ & $2 \mathrm{~S}-66 \mathrm{~S}-39 \mathrm{~S}$ & Slit & $32 / 118$ & 4 \\
\hline 6888A1 & 15 Мay 2009 & 266.5 & $900-90-1200$ & $140-20$ & $3 \mathrm{~N}-65 \mathrm{~S}-43 \mathrm{~S}$ & Slit & $32 / 118$ & 4 \\
\hline 6895A1 & 17 May 2009 & 267.7 & $900-90-1200$ & $140-20$ & $4 \mathrm{~N}-65 \mathrm{~S}-44 \mathrm{~S}$ & Slit & $32 / 118$ & 4 \\
\hline 6949A1 & 02 Jun 2009 & 277.5 & $1300-10-900$ & $150-65$ & $42 \mathrm{~N}-87 \mathrm{~S}$ & Slit & $32 / 118$ & 4 \\
\hline 6956A2 & 04 Jun 2009 & 278.4 & $1300-10-900$ & $150-65$ & $43 N-87 S$ & Slit & $32 / 118$ & 4 \\
\hline 6960A1 & 05 Jun 2009 & 279. & $1300-10-800$ & $144-74$ & $41 \mathrm{~N}-81 \mathrm{~S}$ & Slit & $32 / 118$ & 4 \\
\hline 6967A1 & 07 Jun 2009 & 280.3 & $1300-10-800$ & $145-75$ & $42 \mathrm{~N}-80 \mathrm{~S}$ & Slit & $32 / 118$ & 4 \\
\hline 7045A2\&3 & 29 Jun 2009 & 294. & $3300-8500-7700$ & $64-84$ & $19 S-14 S$ & Slit & $32 / 118$ & 4 \\
\hline $7046 \mathrm{~A} 1 \& 2^{+}$ & 30 Jun 2009 & & & & & & & \\
\hline 7089A3\&4 & 12 Jul 2009 & 301.6 & $2900-9200-8800$ & $70-89$ & $20 \mathrm{~S}-14 \mathrm{~S}$ & Slit & $32 / 118$ & 4 \\
\hline 7090A1\&2 & 12 Jul 2009 & & & & & & & \\
\hline $7122 \mathrm{~A} 2 \& 3$ & 22 Jul 2009 & 307.5 & $2400-9600-9300$ & $73-94$ & $21 \mathrm{~S}-15 \mathrm{~S}$ & Slit & $32 / 118$ & 4 \\
\hline 7123A1\&2 & 22 Jul 2009 & & & & & & & \\
\hline 7292A1 & 08 Sep 2009 & 334.6 & $2500-4000$ & $53-58$ & $58 \mathrm{~S}-40 \mathrm{~S}$ & Slit & $32 / 118$ & 4 \\
\hline 7293A1 & 09 Sep 2009 & 335.2 & $2800-4300$ & $54-59$ & $57 \mathrm{~S}-39 \mathrm{~S}$ & Slit & $32 / 118$ & 4 \\
\hline$\overline{7320 A 2}$ & 17 Sep 2009 & 339.5 & $2100-4100$ & $56-63$ & $67 \mathrm{~S}-52 \mathrm{~S}$ & Slit & $32 / 118$ & 4 \\
\hline 7321A2 & 17 Sep 2009 & 339.5 & $2400-4300$ & $57-63$ & $67 \mathrm{~S}-52 \mathrm{~S}$ & Slit & $32 / 118$ & 4 \\
\hline $7421 \mathrm{~A} 1$ & 16 Oct 2009 & 354.6 & $4200-5600$ & 78 & $74 \mathrm{~S}$ & Slit & $32 / 118$ & 4 \\
\hline $7423 \mathrm{~A} 1$ & 16 Oct 2009 & 354.6 & $200-3000$ & 76 & $76 \mathrm{~S}$ & Slit & $32 / 118$ & 4 \\
\hline 7446A1 & 23 Oct 2009 & 358.1 & $4400-6200$ & 81 & $73 \mathrm{~S}$ & Slit & $32 / 118$ & 4 \\
\hline 7447A3 & 23 Oct 2009 & 358.1 & $4500-6300$ & 80 & $73 \mathrm{~S}$ & Slit & $32 / 118$ & 4 \\
\hline
\end{tabular}

443 Table 2 : List of SPICAM-UV observations used to study the Martian hydrogen corona during the Martian year 29. 444 The orbit number and sequence number of the observations are given in column 1. Column 3 gives the approximate 445 solar longitude Ls at the observation day. Columns 4, 5 and 6 refer to the tangent point of the line of sight. *For 446 these observations solar scattered light is observed at low altitudes on all bands polluting the airglow emissions. 
Only altitudes above $200 \mathrm{~km}$ are considered in these cases.

\section{References}

Bertaux, J-L., O. Korablev, S. Perrier, E. Quémerais, F. Montmessin, F. Leblanc, S. Lebonnois, P. Rannou, F. Lefevre, F. Forget, A. Fedorova, E. Dimarellis, A. Reberac, D. Fonteyn, J-Y. Chaufray, and S. Guibert, SPICAM on Mars Express : Observing modes and overview of UV spectrometer data and scientific results, J. Geophys. Res., 111, E10S90, doi:10.1029/2006JE002690, 2006

Bertucci, C., N. Romanelli, J-Y. Chaufray, D. Gomez, C. Mazelle, M. Delva, R. Modolo, F. Gonzalez-Galindo, and D.A. Brain, Temporal variability of waves at the proton cyclotron frequency upstream from Mars : Implication for Mars distant hydrogen exosphere, Geophys. Res. Lett., 40, 3809-3813, doi:10.1002/grl.50709, 2013

Beth, A., P. Garnier, D. Toublanc, I. Dandouras, and C. Mazelle, (2016), Theory for planetary exospheres : II Radiation pressure effect on exospheric density profiles, Icarus, 266, 423- 432

Bhattacharyya, D., J.T. Clarke, J-L. Bertaux, J-Y. Chaufray, M.Mayyasi, (2015), A strong seasonal dependence in the martian hydrogen exosphere, Geophys. Res. Lett., 42, 8678- 868

Bhattacharyya, D., J.T. Clarke, J-L. Bertaux, J-Y. Chaufray, and M. Mayyasi, (2017) Analysis and modeling of remote observations of the martian hydrogen exosphere, Icarus, 281, 264-280

Bibring, J-P., Y. Langevin, J. Mustard, F. Poulet, R. Arvidson, A. Gendrin, B. Gondet, N. Mangold, P. Pinet, F. Forget, and the OMEGA team, Global Mineralogical and Aqueous history of Mars derived from 
OMEGA/Mars Express data, Science, 312, 400-404, (2006)

Bishop, J., et al., Phyllosilicate diversity and past aqueous activity revealed at Mawrth Vallis, Mars, Science, 321, 830-833, doi : 10.1126/science.1159699, (2008)

Boqueho, V., and P-L. Blelly, Contributions of a multimoment multispecies approach in modeling planetary atmospheres: Example of Mars, J. Geophys. Res., 110, A01313, doi: 10.1029/2004JA010414, 2005

Brinkmann, R.T., Departures from Jeans' escape rate for H and He in the Earth's atmosphere, (1970), Planet. Space Sci., $18,449-478$

Chaffin, M.S., J-Y. Chaufray, I.A.F Stewart, F. Montmessin, N.M. Schneider, and J-L. Bertaux, (2014), Unexpected variability of Martian hydrogen escape, Geophys. Res. Lett., 41, 314- 320, doi: 10.1002/2013GL058578

Chaffin, M.S., J. Deighan, N.M. Schneider, and A.I.F. Stewart, Elevated atmospheric escape of atomic hydrogen from Mars induced by high-altitude water, Nat. Geosc., 10, 174-178, (2017)

Chaffin, M.S., J-Y. Chaufray, J. Deighan, N.M. Schneider, M. Mayyasi, J.T. Clarke, E. Thiemann, S.K. Jain, M.M.J. Crismani, A. Stiepen, F.G. Eparvier, W.E. McClintock, A.I.F. Stewart, G.M. Holsclaw, F. Montmessin, and B.M. Jakosky, Mars H escape rates derived from MAVEN/IUVS Lyman- $\alpha$ brightness measurements and their dependence on model assumptions, J. Geophys. Res: Planets, 123, 2192-2210, doi: 10.1029/2018JE005574, (2018)

Chaufray, J-Y., J-L. Bertaux, F. Leblanc, and E. Quémerais, (2008), Observation of the hydrogen corona with SPICAM on Mars Express, Icarus, 195, 598-613

Chaufray, J-Y., F. Leblanc, E. Quémerais, and J-L. Bertaux, Martian oxygen density at the exobase deduced from OI 130.4 nm observations by SPICAM on Mars Express, J. Geophys. Res., 114, E02006, doi:10.1029/2008JE003130, 2009

Chaufray, J-Y., J-L. Bertaux, E. Quémerais, E. Villard, F. Leblanc, Hydrogen density in the dayside venusian exosphere derived from Lyman- $\alpha$ observations by SPICAV on Venus Express, Icarus, 217, 767-778, 2012

Chaufray, J-Y., F. Gonzalez-Galindo, F. Forget, M.A. Lopez-Valverde, F. Leblanc, R. Modolo, and S. Hess, (2015a), Variability of the hydrogen in the martian upper atmosphere as simulated by a 3D atmosphere-exosphere coupling, Icarus, 245, 282-294

Chaufray, J-Y., J-L. Bertaux, F. Leblanc, E. Quémerais, and S. Sulis, (2015b), Observations of the nightside venusian hydrogen corona with SPICAV/VEX, Icarus, 262, 1-8 
Chaufray, J-Y., F. Gonzalez-Galindo, F. Forget, M.A. Lopez-Valverde, F. Leblanc, R. Modolo, and S. Hess, (2017), Reply to comment « On the hydrogen escape: Comment to Variability of the hydrogen in the Martian upper atmosphere as simulated by a 3D atmosphere-exosphere coupling by J-Y Chaufray et al." by V. Krasnopolsky, Icarus, 281, 262, doi : 10.1016/j.icarus.2017.07.013

Chaufray, J-Y., R.V. Yelle, F. Gonzalez-Galindo, F. Forget, M. Lopez-Valverde, F. Leblanc, R. Modolo, (2018), Effect of the lateral exospheric transport on the horizontal hydrogen distribution at the exobase of Mars, J. Geophys. Res., 123, 4241-4254, doi : 10.1002/2017JA025163

Clancy, R.T., B.J. Sandor, M.J. Wolff, P.R. Christensen, M.D. Smith, J.C. Pearl, B.J. Conrath, and R.J. Wilson, An intercomparison of ground-based millimeter, MGS TES, and Viking atmospheric temperature measurements: Seasonal and interannual variability of temperatures and dust loading in the global Mars atmosphere, J. Geophys. Res., 105, 9553-9571, 2000

Clarke, J. T., J-L. Bertaux, J-Y. Chaufray, G.R. Gladstone, E. Quémerais, J.K. Wilson, and D. Bhattacharyya, A rapid decrease of the hydrogen corona of Mars, Geophys. Res. Lett., 41, 8013-8020, 2014

Clarke, J.T., M. Mayyasi, D. Bhattacharyya, N.M. Schneider, W.E. McClintock, J.I. Deighan, A.I.F. Stewart, J-Y. Chaufray, M.S. Chaffin, S.K. Jain, A. Stiepen, M. Crismani, G.M. Holsclaw, F. Montmessin, and B. Jakosky, Variability of D and H in the Martian upper atmosphere observed with the MAVEN IUVS echelle channel, J. Geophys. Res., 122, 2336-2344, doi: 10.1002/2016JA023479

Emerich, C., P. Lemaire, J-C. Vial, W. Curdt, U. Schüle, K. Wilhelm, A new relation between the central spectral solar H I Lyman $\alpha$ irradiance and the line irradiance measured by SUMER/SOHO during the cycle 23., Icarus, $178,429-433,(2005)$

Fedorova, A., J-L. Bertaux, D.Betsis, F. Montmessin, O. Korablev, L. Maltagliati, and J. Clarke, Water vapor in the middle atmosphere of Mars during the 2007 global dust storm, Icarus, 300, 440, 2018

Forget, F., F. Hourdin, R. Fournier, C. Hourdin, O. Talagrand, M. Collins, S.R. Lewis, P.L. Read, J-P. Huot, Improved general circulation models of the Martian atmosphere from the surface to above $80 \mathrm{~km}$, J. Geophys. Res., 104, 24,155-24,175, 1999

Fox J.L., M. Benna, P.R. Mahaffy, and B.M. Jakosky, Water and water ions in the Martian thermosphere/ionosphere, Geophys. Res. Lett., 42, 8977-8985, doi: 10.1002/2015GL065465, (2015)

Gladstone, G.R., Auroral resonance line radiative transfer, J. Geophys. Res., 97, 1377-1387, 1992 
Gonzalez-Galindo, F., F. Forget, M.A. Lopez-Valverde, M. Angelats i Coll, and E. Millour, A ground-to-exosphere Martian general circulation model: 1 Seasonal, diurnal, and solar cycle variation of thermospheric temperatures, J. Geophys. Res., 114, E04001, doi:10.1029/2008JE003246, 2009

Gonzalez-Galindo, F., M.A. Lopez-Valverde, F. Forget, M. Garcia-Comas, E. Millour, and L. Montabone, (2015), Variability of the Martian thermosphere during eight Martian years as simulated by a ground-to-exosphere global circulation model, J. Geophys. Res., 120, 2020-2035, doi:10.1002/2015JE004925

Halekas, J. S. (2017), Seasonal variability of the hydrogen exosphere of Mars, J. Geophys. Res. Planets, 122, doi:10.1002/2017JE005306

Heavens et al, Hydrogen escape from Mars enhanced by deep convection in dust storms, Nature Astronom, 2, 126$132(2018)$

Hynek, B.M., M. Beach, and M.R.T. Hoke, Updated global map of Martian valley networks and implications for climate and hydrologic processes, J. Geophys. Res., 115, E09008, doi: 10.1029/2009JE003548, (2010)

Jakosky, B. et al., Loss of the Martian atmosphere to space: Present-day loss rates determined from MAVEN observations and integrated loss through time, Icarus, 315, 146-157, (2018)

Krasnopolsky, V., Mars' upper atmosphere and ionosphere at low, medium, and high solar activities: Implications for evolution of water, J. Geophys. Res., 107(E12), 5128, doi: 10.1029/2001JE001809, 2002

Krasnopolsky, V., Solar activity variations of thermospheric temperatures on Mars and a problem of CO in the lower atmosphere, Icarus, 207, 638-647, (2010)

Krasnopolsky, V., On the hydrogen escape from Mars: comments to "Variability of the hydrogen in the Martian upper atmosphere as simulated by a 3D atmosphere-exosphere coupling” by JY Chaufray et al., Icarus, 281, 262-263, (2017)

Krasnopolsky, V.A., Photochemistry of water in the martian thermosphere and its effect on hydrogen escape, Icarus, $\underline{321,62-70,2019}$

Lasue, J., N. Mangold, E. Hauber, S. Clifford, W. Feldman, O. Gasnault, C. Grima, S. Maurice, and O. Mousis, Quantitative assessments of the Martian hydrosphere, Space Sci. Rev., 174, 155-212, (2013)

Leblanc, F., J-Y. Chaufray, J. Lilensten, O. Witasse, and J-L. Bertaux, Martian dayglow as seen by the SPICAM UV spectrograph on Mars Express, J. Geophys. Res., 111, E09S11, doi: 10.1029/2005JE002664, 2006

Lee, J.S., Refined Monte Carlo method for simulating angle-dependent partial frequency redistributions, Astrophys. 
J., 255, 303-306, 1982

Lopez-Valverde, M.A. et al., Investigation of the Mars upper atmosphere with ExoMars Trace Gas Orbiter, Space Sci. Rev., 214:29, doi: 10.1007/s11214-017-0463-4, (2018)

Maltagliati, L., F. Montmessin, O. Korablev, A. Fedorova, F. Forget, A. Määtänen, F. Lefèvre, and J-L. Bertaux, (2013) Annual survey of water vapor vertical distribution and water- aerosol coupling in the martian atmosphere observed by SPICAM/Mex solar occultations, Icarus, 223, 942-962

Mayyasi, M., D. Bhattacharyya, J. Clarke, A. Catalano, M. Benna, P. Mahaffy, E. Thiemann, C. Lee, J. Deighan, J. Sonal, and 9 co-authors, (2018), Significant space weather impact on the escape of hydrogen from Mars, Geophys. Res. Lett., 45, 8844

Montabone, L., F. Forget, E. Millour, R.J. Wilson, S.R. Lewis, B. Cantor, D. Kass, A. Kleinbohl, M.T. Lemmon, M.D. Smith, M.J. Wolff, Eight-year climatology of dust optical depth on Mars, Icarus, 251, 65-95, 2015

Montmessin, F., F. Forget, P. Rannou, M. Cabane, R. M. Haberle, Origin and role of water ice clouds in the Martian water cycle as inferred from a general circulation model, J. Geophys. Res., 109, E10004, doi : 10.1029/2004JE002284, 2004

Montmessin, F., O. Korablev, F. Lefevre, J-L. Bertaux, A. Fedorova, A. Trokhimovskiy, J-Y. Chaufray, G. Lacombe, A. Reberac, L. Maltagliati, Y. Willame, S. Guslyakova, J-C. Gerard, A. Stiepen, D. Fussen, N. Mateshvili, A. Maatanen, F. Forget, O. Witasse, F. Leblanc, A.C. Vandaele, E. Marcq, B. Sandel, B. Gonder, N. Schneirder, M Chaffin, N. Chapron, SPICAM on Mars Express: A 10 year in-depth survey of the Martian atmosphere, Icarus, 297, 195-216, 2017

Navarro, T., J.-B. Madeleine, F. Forget, A. Spiga, E. Millour, F. Montmessin, and A. Määttänen. Global climate modeling of the Martian water cycle with improved microphysics andadiatively active water ice clouds. Journal of Geophysical Research (Planets), 119:1479- 1495, 2014

Orosei, R., R.L. Jordan, D.D Morgan, M. Cartacci, A. Cicchetti, F. Duru, D.A. Gurnett, E. Heggy, D.L. Kirchner, R. Noschese, W. Kofman, A. Masdea, J-J. Plaut, R. Seu, T.R. Watters, G. Picardi, Mars Advanced Radar for subsurface and ionospheric sounding (MARSIS) after nine years of operation : A summary, Planet Space Sci., $112,98-114,(2015)$

Orosei, R., S.E. Lauro, E. Pettinelli, A. Ciccheti, M. Coradini, B. Cosciotti, F. Di Paolo, E. Flamini, E. Mattei, M. Pajola, and 12 co-auhors, Radar evidence of subglacial liquid water on Mars, Science, 361, 490, (2018) 
Quémerais, E., Angle dependent partial frequency redistribution in the interplanetary medium at Lyman- $\alpha$, Astron. Astrophys., 358, 353-367, 2000

Rahmati, A., D.E. Larson, T.E. Cravens, R.J. Lillis, J.S. Halekas, J.P. McFadden, D.L. Mitchell, E.M.B. Thiemann, J.E.P. Connerney, P.A. Dunn, C.O. Lee, F.G. Eparvier, G.A.DiBraccio, J.R. Espley, J.G. Luhmann, C. Mazelle, and B.M.. Jakosky, Seasonal variability of neutral escape from Mars as derived from MAVEN pickup ion observations, J. Geophys. Res. : Planets, 123, 1192-1202, 10.1029/2018JE005560, (2018)

Romanelli, N. C. Mazelle, J-Y. Chaufray, K. Meziane, L. Shan, S. Ruhunusiri, J.E.P. Connerney, J. R. Espley, F. Eparvier, T.E. Thiemann, et al., 2016, Proton cyclotron, waves occurrence rate upstream from Mars observed by MAVEN : Associated variability of the Martian upper atmosphere, J. Geophys. Res., 121, 11,113-11,128, doi : 10.1002/2016JA023270

Rottman, G.J., N.W. Thomas; W. McClintock, SORCE solar UV irradiance results, Adv. Space Sci., 37, 201-208, (2006)

Shaposhnikov, D.S., A.S. Medvedev, A.V. Rodin, and P. Hartogh, Seasonal water "pump" in the atmosphere of Mars: Vertical transport to the thermosphere, Geophys. Res. Lett., 46, 4161-4169, (2019)

Shizgal, B., and R. Blackmore, A collisional kinetic theory of a plane parallel evaporating planetary atmosphere, Planet. Space Sci., 34, 279-291, (1986).

Terada, K., N. Terada, H. Shinagawa, H. Fujiwara, Y. Kasaba, K. Seki, F. Leblanc, J-Y. Chaufray, and R. Modolo, A full-particle Martian upper thermosphere-exosphere model using the DSMC method, J. Geophys. Res., 121, 1429-1444, doi: 10.1002/2015JE004961,2016

Vandaele, A.C, O. Korablev, F. Daerden, et al., Martian dust storm impact on atmospheric H2O and D/H observed by ExoMars Trace Gas Orbiter, Nature, 568, 52, (2019)

Vidal-Madjar, A., and J-L. Bertaux, A calculated hydrogen distribution in the exosphere, Planet Space Sci., 20, 11471162,1972

Yamauchi, M., T. Hara, R. Lundin, E. Dubinin, A. Fedorov, J-A. Sauvaud, R.A. Frahm, R. Ramstad, Y. Futaana, M. Holmstrom, S. Barabash (2015), Seasonal variation of martian pick-up ions: Evidence of breathing exosphere, Planet. \& Sp. Sci., 119, p. 54 


\title{
Study of the hydrogen escape rate at Mars during Martian years 28 and 29 from comparisons between SPICAM/Mars Express observations and GCM-LMD simulations
}

\author{
Chaufray, J-Y ${ }^{1}$., F. Gonzalez-Galindo 2 , M.A. Lopez-Valverde 2 , F. Forget ${ }^{3}$, E. Quémerais ${ }^{1}$, J-L. Bertaux ${ }^{1}$, F. \\ Montmessin ${ }^{1}$, M. Chaffin ${ }^{4}$, N. Schneider ${ }^{4}$, J.T. Clarke ${ }^{5}$, F. Leblanc ${ }^{1}$, R. Modolo ${ }^{1}$, R.V. Yelle ${ }^{6}$ \\ ${ }^{1}$ LATMOS,CNRS, Guyancourt, France, ${ }^{2} I A A$, CSIC, Granada, Spain, ${ }^{3} L M D, C N R S$, Paris, France, ${ }^{4} L A S P$, \\ Boulder, CO, USA, ${ }^{5}$ Boston University, Boston, MA, USA, ${ }^{6}$ University of Arizona, Tucson, AZ, USA
}

Abstract

We have simulated the 3D atomic hydrogen density in the Martian upper atmosphere and associated Jeans escape rate during Martian years 28 and 29. The coronal Lyman- $\alpha$ brightness is computed using a 3D radiative transfer model which accounts for the monthly average hydrogen density for these two years and is compared to a large set of observations by Mars Express/SPICAM. The simulated brightness is generally in good agreement with the observations for $\mathrm{Ls}<230^{\circ}$ and Ls $>330^{\circ}$ for Martian year 28 and Ls $<270^{\circ}$, Ls $>340^{\circ}$ for Martian year 29 , but the model strongly underestimated the brightness for $230<\mathrm{Ls}<330^{\circ}$ for Martian year 28 and $270<\mathrm{Ls}<340^{\circ}$ for Martian year 29. In these simulations the transport of water vapor contributes to the production of hydrogen at high altitudes during southern summer. A possible explanation for the model discrepancy is an underestimate of this water transport, associated with an underestimate of the hygropause altitude and/or an underestimate of the supersaturation of the mesosphere. Considering this discrepancy, we estimate the hydrogen escape rate during these two Martian years to vary by almost two orders of magnitude, between $\sim 10^{25}$ to $6 \times 10^{26} \mathrm{~s}^{-1}$ (equivalent to a global layer of water $\sim 33$ to $2000 \mathrm{~mm}$ deep every billion years), in agreement with the seasonal variations estimated directly from the fit of the SPICAM observations during the Martian year 28 by Chaffin et al. (2014). Our analysis suggests that episodic dust storms and associated enhancements at high altitude near perihelion are a major factor in the $\mathrm{H}$ escape estimates averaged over one martian year or longer periods, but the accumulated water lost at this rate for 4 billions years is much lower than the amount of water needed to form the flow channels observed on Mars.

1) Introduction

Several geologic and mineralogic observations indicate that Mars was not always as dry as it is today (e.g. Bibring et al. 2006, Bishop et al. 2008, Hynek et al. 2010). The fate of the water is still unknown, but substantial amounts could have escaped into the interplanetary medium in the form of atomic hydrogen (Jakosky et al. 2018). So, understanding 
of how water currently escapes from Mars is necessary to investigate the long-term evolution of the Martian climate.

Recent observations suggest that the amount of exospheric hydrogen at Mars has important seasonal variations, with a larger abundance during southern summer (Chaffin et al. 2014, Clarke et al. 2014, 2017, Bhattacharyya et al. 2015). This increase of the hydrogen density should be associated with an increase of the hydrogen Jeans escape. These seasonal variations have a large impact on the plasma environment of Mars (Bertucci et al. 2013, Yamauchi et al. 2015, Romanelli et al. 2015, Halekas 2017, Rahmati et al. 2018), and could be driven by large amounts of water vapor in the mesosphere, as observed by Mars Express/SPICAM (Maltagliati et al. 2017, Fedorova et al. 2018), during dust storm season (Chaffin et al. 2017, Heavens et al. 2018). Using the Global Circulation Model of Laboratoire de Météorologie Dynamique (GCM-LMD) (Forget et al. 1999, Gonzalez-Galindo et al. 2009, 2015), we were able to simulate seasonal variations of the hydrogen escape, but with a lower range than derived from observations (Chaufray et al. 2015a). In order to better characterize the hydrogen exosphere of Mars and the variability of hydrogen escape, we performed a detailed study of the Martian years 28 and 29, and compared the simulated brightness to a large SPICAM Lyman- $\alpha$ dataset during the same two years. The year number used in this study follows the convention chosen by Clancy et al. (2000) with April $111955\left(\mathrm{Ls}=0^{\circ}\right)$ chosen as the beginning of the Martian year 1.

The data used in this study is described in section 2. In section 3, we present our 3D simulated Lyman- $\alpha$ emissions corresponding to the Martian years 28 and 29, which are obtained by coupling several models. The comparisons between the observed and simulated Lyman- $\alpha$ profiles are presented in section 4 and the possible origin of the discrepancy is discussed in section 5 , followed by conclusions in the last section.

\section{2) Observations}

The Spectroscopy for Investigation and Characterization of the Atmosphere of Mars (SPICAM) is a dual ultraviolet and infrared spectrometer aboard Mars Express (Bertaux et al. 2006, Montmessin et al. 2017). The ultraviolet channel uses an optical entrance of $4 \mathrm{~cm}$ diameter feeding an off-axis parabolic mirror which focuses the observed scene at the focal plane. The instantaneous field of view (IFOV) is limited at the focal point of the parabolic mirror by a 50 $\mu \mathrm{m}$ wide retractable slit that extends over an angular aperture of $2.8^{\circ}$, equivalent to about two pixels on the UV detector. In the upper most portion of the slit, a 10 times wider aperture allows for the observations of fainter sources at the expense of degraded spectral resolution. 
In principle, SPICAM can record 288 spatially resolved spectra along the slit. However, to save data volume transmission, only 5 spectra are transmitted each second. For all the observations presented here, the spectra are the sum of 32 individual CCD line spectra forming 5 adjacent spatial bins of $0.32^{\circ}$ each, starting from the CCD line $\mathrm{Y}_{0}$. The wavelength range of the UV channel is $118-320 \mathrm{~nm}$ and includes the strong hydrogen Lyman- $\alpha$ emission line at $121.6 \mathrm{~nm}$. This emission line is produced by resonant scattering of solar photons by hydrogen atoms in the Martian upper atmosphere. Such emission is observed over several Martian radii as a signature of the extended hydrogen exosphere of Mars (Chaufray et al. 2008).

We selected several observations obtained during the Martian years 28 (from 22 Jan 2006 to 9 Dec 2007) and 29 (from 10 Dec 2007 to 26 Oct 2009) performed by the UV spectrograph SPICAM on Mars Express, including those studied by Chaffin et al. (2014). During this period the UV channel exhibited an anomalous image intensifier behavior, leading to sporadic changes of the high voltage during a sequence of observations (Montmessin et al. 2017). We selected by visual inspection the observations least affected by this behavior. A cleaning procedure has been developed to flag the UV channel spectra affected by these high voltage spikes (Montmessin et al. 2017). All these flagged spectra have been removed from use in this study.

The list of observations used for this study is summarized in Tables 1 and 2 in theAppendix.

Some of the observations were performed without the slit, while others were performed with the slit. Also, the $5 \mathrm{CCD}$ spatial bands (corresponding to a binning of 32 individual, consecutive lines) transmitted each second can differ between observations. Three different configurations are used in the observations presented here. The first configuration corresponds to an observation with the slit and starts from $\mathrm{CCD}$ line $\mathrm{Y}_{0}=23$, the second configuration also corresponds to an observation with the slit, but starts from CCD line $\mathrm{Y}_{0}=118$, and the third configuration corresponds to an observation without the slit, starting from CCD line $\mathrm{Y}_{0}=118$.

For the first configuration, all the transmitted bands correspond to the part of the CCD in front of the narrow part of the slit, so we chose band 5, which is close to the center of the CCD (lines 118- 149). For the second configuration, the last two bands correspond to the part of the CCD in front of the large part of the slit. The signal to noise ratio is generally better, and straylight near 132 and $143 \mathrm{~nm}$ discussed by Leblanc et al. (2006) and Chaufray et al. (2009) is not detected in those bands. Therefore, we chose band 4 (lines 214-245), which is more sensitive than band 5 by about $20 \%$ (Fig. 1a). For the last configuration without the slit, we chose band 1, which corresponds to the same CCD lines as configuration 1 (lines 118-149), but is slightly different (by about 5\%) than the derived brightness from band 

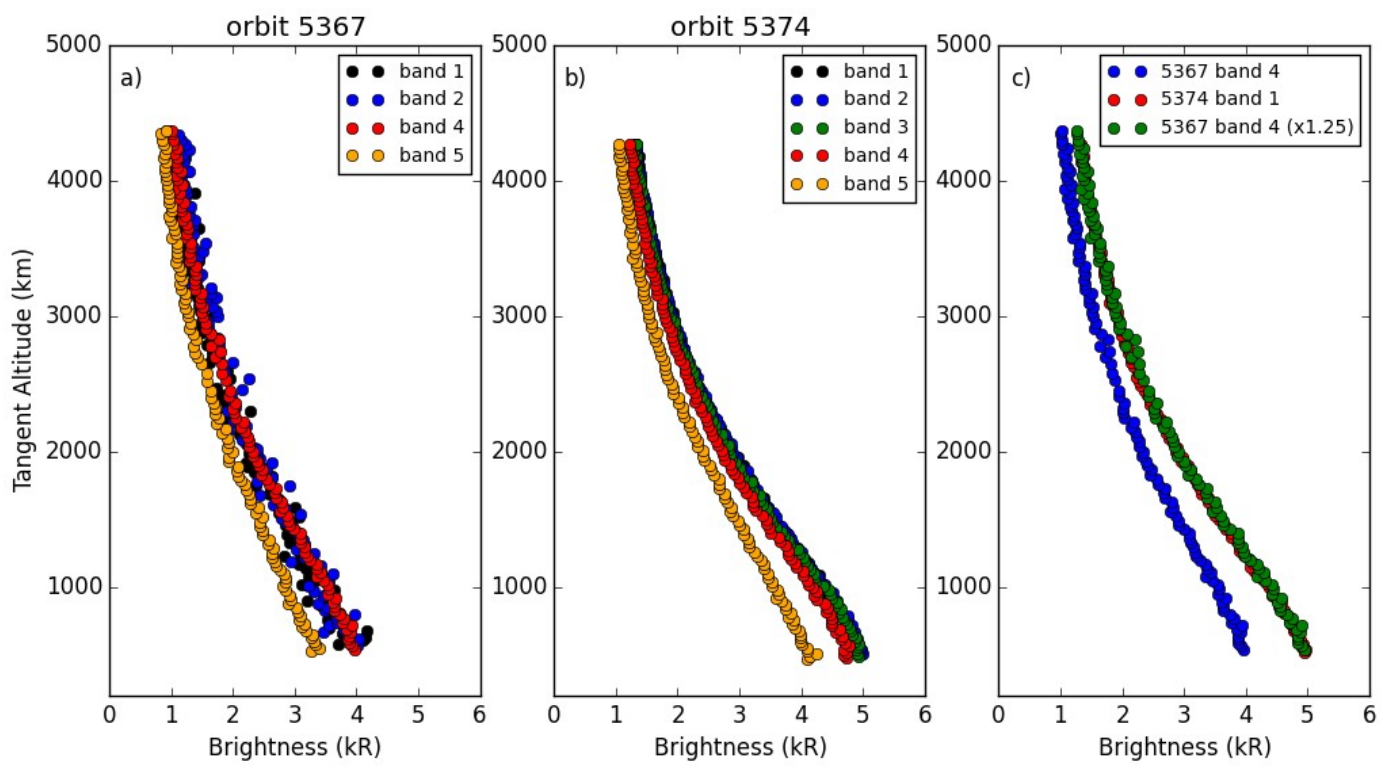

4 (lines 214-245) (Fig. 1b). As shown in Fig. 1, the signal to noise ratio of the derived brightness profile is very good and trying to improve it by using the 5 transmitted bands will not change our results since the calibration uncertainty is larger than the standard deviation of the measurement.

Fig. 1 : a) Brightness profiles derived from Mars Express orbit 5367 with the slit over the first 2 and last 2 transmitted bands of the CCD. b) Brightness profiles derived from Mars Express orbit 5374 without the slit over the 5 transmitted bands. c) Comparison between the profiles derived from the two orbits over the bands used in this study considering a 1.25 factor for the observations with the slit

Observations with very similar geometries (orbits 5367 and 5374 for example, Fig. 1) obtained with and without the slit, indicate a systematic $\sim 20 \%$ underestimate of the derived brightness with the slit compared to the observations without the slit (Fig. 1b). 5\% could be attributed to the different CCD lines as suggested by Fig. 1b. The rest of the difference could come from the method used to integrate the spectral line, neglecting the extended spectral wings, or in a continuum background subtraction. Therefore, to summarize, the brightness measured by configuration 3 is used as a reference, and a correction by $20 \%$ is applied to the brightness measured in configuration 1 and a correction of $25 \%$ is applied to the brightness measured by configuration 2. Examples of Lyman- $\alpha$ vertical profiles are displayed in Fig. 1.

The spectral shape of the Lyman- $\alpha$ line measured for each configuration is displayed in Fig. 2. For the first two configurations, the brightness is computed by integration over the line profile after subtraction of a residual linear background (Chaufray et al. 2008). For the third (slitless) configuration, the average number of counts per pixel is computed and converted to Rayleighs using the solid angle defined by one pixel. All spectra have been corrected for 


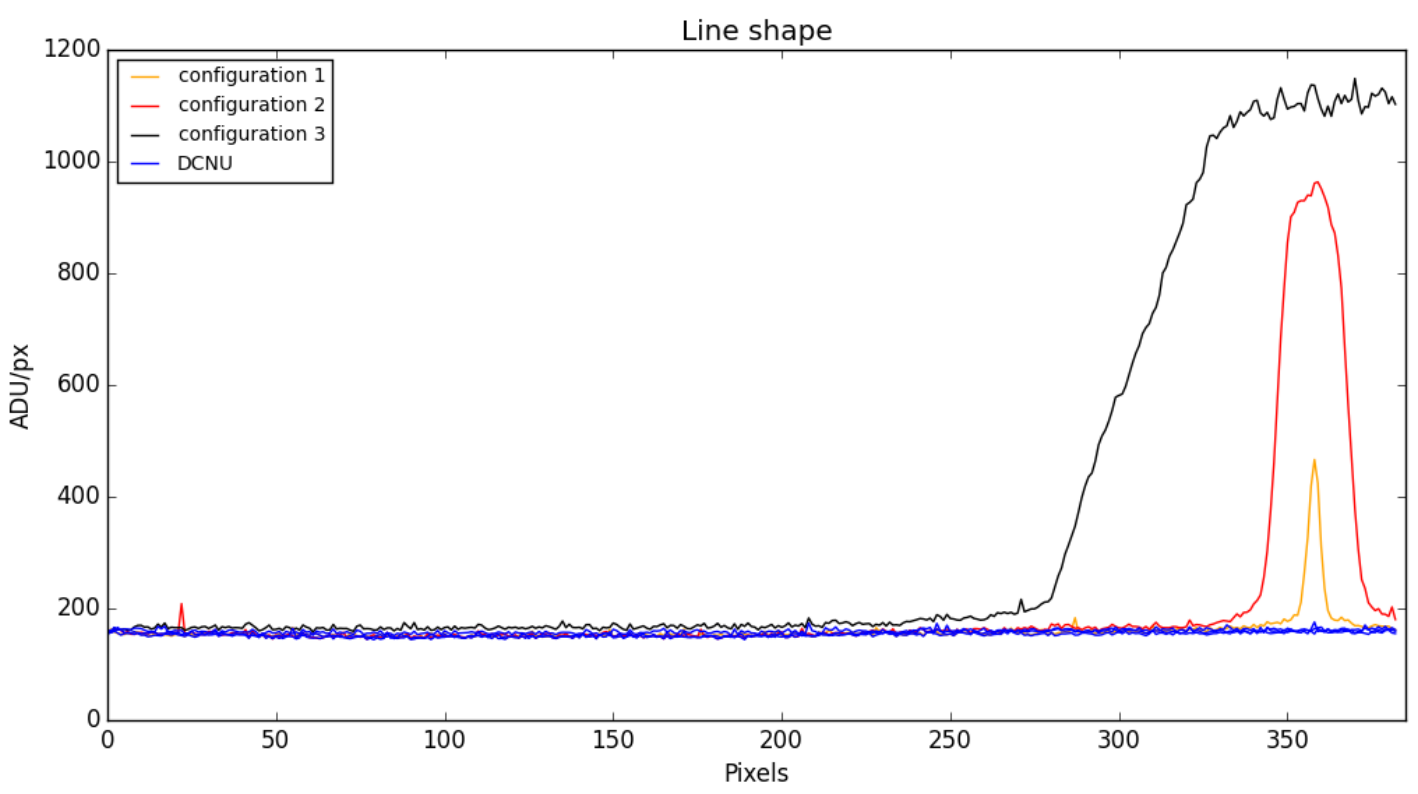

Fig. 2 Spectral shape of the Lyman-a line measured for the three configuration used in the paper. These spectral profile correspond to the average observation between 2000 and $2200 \mathrm{~km}$ from observation 5367 (narrow slit : configuration 1 and large slit : configuration 2) and observation 5374 (no slit : configuration 3). A sapphire filter is glued above the CCD MgF 2 input window, preventing Lyman- $\alpha$ photons to reach pixels $<250$ without slit (Bertaux et al. 2006). With the slit, the linewidth is constrained by the slit size. The Dark charge non-uniformity (DCNU) is also indicated in blue and is very similar for these three measured spectra.

Such a systematic uncertainty is in the range of the absolute calibration of the instrument from star observations (Montmessin et al. 2017). Therefore, in this study we focus on systematic differences between the observations and the simulations which are $>25 \%$, as well as the differences in the shape of the observed and simulated vertical variations.

\section{3) Models}

To simulate the hydrogen corona of Mars during Martian years 28 and 29, we used three models. The first model is the GCM-LMD describing the Martian atmosphere from the surface to the exobase (Forget et al. 1999, GonzalezGalindo et al. 2009, 2015), including the hydrogen in the thermosphere (Chaufray et al. 2015a, 2018). The second model is an exospheric model considering nonuniform conditions at the exobase (hydrogen density and temperature from the GCM-LMD) to derive the hydrogen density in the exosphere, based on the approach of Vidal-Madjar and Bertaux 1972. The last model is a 3D Monte Carlo radiative transfer model used to simulate the resonance Lyman- $\alpha$ 
spectral volume emission rate (Chaufray et al 2015b). The Lyman- $\alpha$ brightness is computed by formal integration of the radiative transfer equation using simulated volume emission rates for the specific geometry (spacecraft position and line of sight direction) of each individual observation listed in Tables 1 and 2 in Appendix A. The simulated brightness is directly proportional to the solar flux at the center of the solar Lyman- $\alpha$ line. To derive it, we used the solar brightness between 121-122 nm measured by SORCE (Rottman et al. 2006) rescaled to Mars distance, with accounting for the different ecliptic longitudes of Earth and Mars as seen from the Sun. The brightness at the center of the line is derived from the empirical relation given by Emerich et al. (2005). The variability of the solar flux between 121 and $122 \mathrm{~nm}$ measured by SORCE during the full period is $\sim 10 \%$, therefore uncertainty on the flux at Mars, due to the different ecliptic longitudes of Mars and Earth should be at most $\sim 10 \%$ but could differ from one observation to the other. Due to the large CPU time required by the Monte Carlo radiative transfer simulations, it is not possible to simulate the Martian Lyman- $\alpha$ volume emission rate with every GCM-LMD time step. In this study, we consider only monthly average hydrogen for the exosphere and corona. This is another source of uncertainty in the simulated brightness that prevents us from discussing differences less than a few tenths percent in the brightness comparisons. This resolution is enough to discuss the seasonal variations that are important, as observed from several missions (Chaffin et al. 2014, 2018, Clarke et al. 2014, Battacharyya et al. 2015, 2017).

\section{1) Thermospheric density}

The GCM-LMD is a 3D model of the Martian atmosphere from the surface to the exobase. It is composed of a 3D dynamics core which solves the fluid equations over a sphere and a physical core, describing all the physical processes computed for each column. In all the simulations presented below, the horizontal resolution is $5.625^{\circ}$ in longitude and $3.75^{\circ}$ in latitude. The dynamical time step $\delta \mathrm{t}$ is $1 \mathrm{sol} / 960 . \sim 1.5$ minute, and the physics time step is $\Delta \mathrm{t}$ $=5 \delta \mathrm{t} \sim 7.5$ minutes. More details on the GCM-LMD can be found in Forget et al. (1999) and Gonzalez-Galindo et al. (2009, 2015). As noted in Chaufray et al. (2015a) the vertical resolution used in the molecular diffusion (2 km) can lead to an uncertainty of a few tenths in the hydrogen density above $200 \mathrm{~km}$, depending on the season and martian year. Because we will focus on large differences between the simulated brightness and observed brightness, this uncertainty as a monthly average exosphere, should not change our conclusion.

In these simulations, we account for the daily variations of the solar flux during the Martian year 28 and 29 as computed by Gonzalez-Galindo et al. (2015). We also consider the dust scenarios of these years derived by 
Montabone et al. (2015). We do not include the microphysical processes controlling the supersaturation presented in Navarro et al. (2014), as these results, and especially the hydrogen density in the Martian thermosphere, depend on model parameters not well constrained by the observations. These simulations will be discussed in section 5 . We also do not include the coupling with the exospheric ballistic transport developed by Chaufray et al. (2018). This coupling can't be performed for a full Martian year because of the large CPU time needed. The first few studies we performed indicate that the effect on the brightness should not be greater than a few tens of percent, and therefore, the differences discussed in section 5 should still be present with this coupling. A study of the local time variations would require such a coupling. Therefore, in this paper we will focus on the seasonal variation of the hydrogen corona and escape. We only consider the hydrogen Jeans escape, computed assuming a Maxwellian distribution at the upper level. Shizgal and Blackmore (1986) have shown that the depletion of energetic atoms could reduce the real escape rate by a factor $\sim 0.57$ for an exospheric temperature of $230 \mathrm{~K}$ and 0.53 for an exospheric temperature of $310 \mathrm{~K}$, We also neglect the non-thermal escape processes which should be not very efficient for atomic hydrogen compared to the thermal escape rate (Krasnopolsky 2010). The non-thermal escape of molecular hydrogen was discussed in Krasnopolsky (2017) and Chaufray et al. (2017) and is not included in these simulations.

The simulated escape flux for the Martian years 28 and 29, as well as the escape flux derived from the monthly average conditions for Martian year 28 are displayed on Fig.2. These variations are close to the simulated escape rates for solar minimum conditions by Chaufray et al. (2015a; 2018) as expected for these Martian years. 


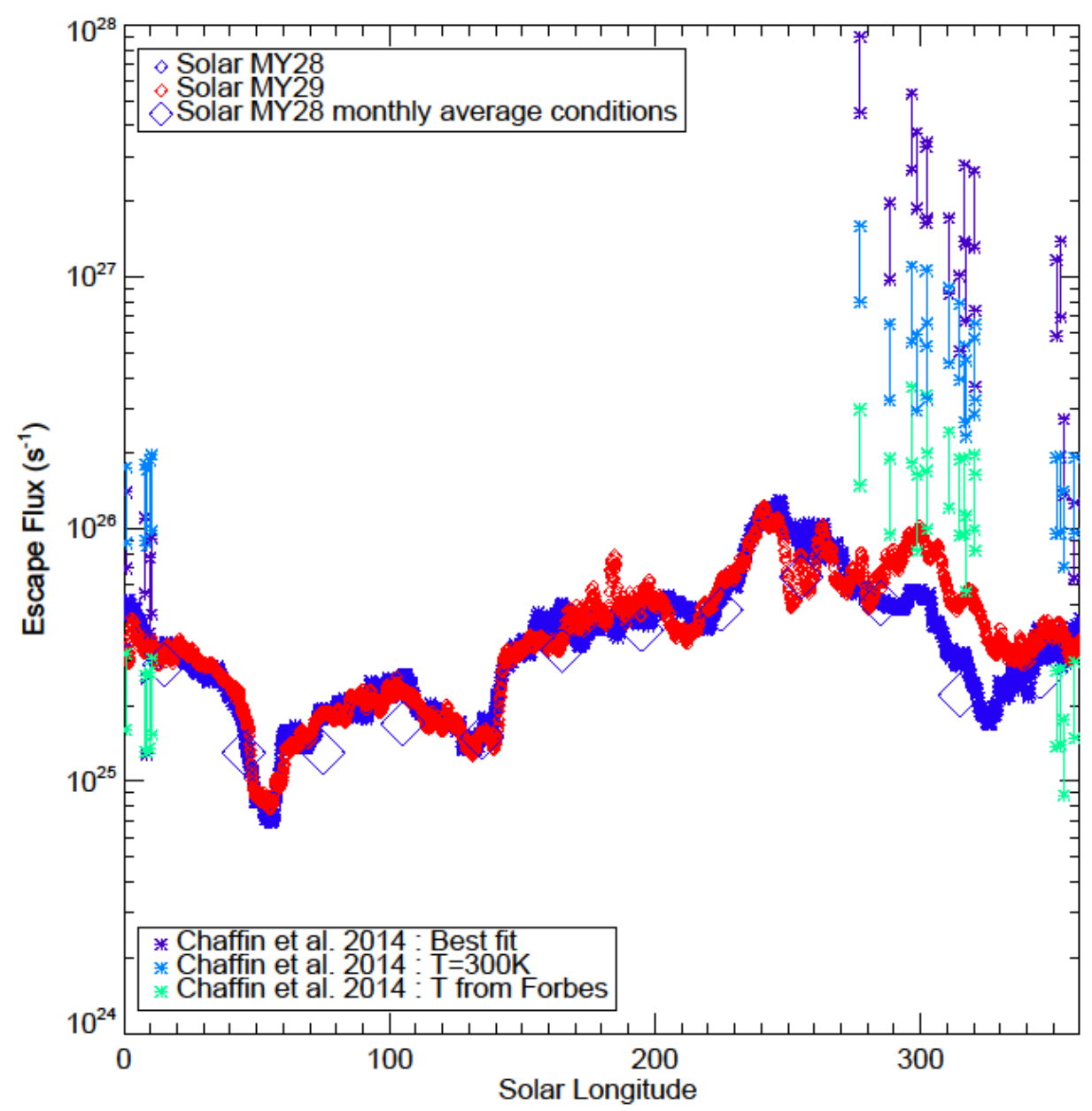

Fig. 3 : Jeans hydrogen escape rate simulated for the Martian years 28 (blue) and 29 (red) with the GCM-LMD. The escape rate derived from the monthly average upper atmosphere are also indicated by the blue diamonds. The escape rates derived from SPICAM fit (from observations between end of Martian year 28, beginning of Martian year 29) using different assumptions by Chaffin et al. 2014 are also displayed. The escape flux (at $\left./ \mathrm{cm}^{2} / \mathrm{s}\right)$ was converted to escape rate (at/s). For this conversion, we assume either a uniform flux at the exobase or a uniform flux at the dayside exosbase and no escape flux at the nightside, leading to the range given by each vertical line.

\section{2) Exospheric density}

The hydrogen density is extended to the exosphere (above $200 \mathrm{~km}$ ), by solving the Liouville equations from nonuniform conditions at the exobase (Vidal-Madjar and Bertaux 1972). We consider Maxwellian velocity distribution functions at the exobase using the temperature and the hydrogen density computed from the LMD-GCM reinterpolated at $200 \mathrm{~km}$ as done in our previous simulations (Chaufray et al. 2015a, 2018). The real distribution at the exobase should differ from a Maxwellian distribution. Due to the escaping particles, the Maxwellian distribution is truncated at velocity larger than the escape velocity (Shizgal and Blackmore 1986, Boqueho et al. 2005, Terada et al., 2016). However, this truncation should not affect the ballistic distribution with a velocity lower than the escaping 
velocity but only reduce the escape rate (Brinkmann, 1970). The Direct Simulation Monte Carlo method used by Terada et al. (2016) shows that the ballistic hydrogen population is very close to the Maxwellian distribution at the local temperature (see Fig 8 b in Terada et al. 2016).

In the computation of the exospheric hydrogen density we neglect ionization loss and the effect of solar radiation pressure that should be important at a few Martian radii above the altitudes scanned by the missions and the optically thick region of the hydrogen corona (Beth et al.2014).

\section{3) Radiative transfer model for resonance line}

The computation of the Lyman- $\alpha$ brightness is done in two steps, as done by numerous models (e.g., Gladstone 1992). In a first step, we use a 3D Monte Carlo radiative transfer model to compute the $3 \mathrm{D}$ volume emission rate from $80 \mathrm{~km}$ to $50,000 \mathrm{~km}$. This radiative transfer model is a 3D version of the model used by Chaufray et al. (2012, 2015b) to study the hydrogen corona of Venus, which accounts for angle-dependent partial frequency redistribution (Lee 1982) and describes the spectral line from -5 to +5 Doppler width (at the maximum temperature at the exobase, i.e., $\sim \pm 40 \mathrm{~mA})$.

The brightness is then computed in a second step, rescaling the volume emission rate to the solar flux at Lyman- $\alpha$ at the time of the observations and accounting for the geometry of each individual observation from Mars Express (Chaufray et al. 2008). Twenty-four 3D volume emission rates, monthly average, corresponding to Martian months of the years 28 and 29 have been computed. To reduce the CPU time, we did not simulate every line of sight for each observation, but instead averaged several lines of sight (between 5 and 20) and compared the simulated brightness of the average line of sight with the observed average brightness.

The interplanetary brightness emission is estimated for each individual observation using a 3D radiative transfer model of the interplanetary emission (Quémerais 2000). The possible uncertainty on the brightness should be small ( 100R) compared to the brightness of the Martian hydrogen corona and will not change the conclusion of this study.

\section{4) Effect of ballistic transport in the Martian exosphere}

The effect of ballistic transport on the hydrogen density near the exobase of Mars has been recently studied (Chaufray et al. 2018). This coupling has not been included in this study due to the large CPU needed to perform such coupled simulations. Our first estimates indicate that considering this coupling has a small effect on the 
simulated brightness. The Lyman- $\alpha$ emission line is optically thick below few thousands kilometers in altitude (Bhattacharyya et al. 2017) and therefore is sensitive to global conditions and not too significantly affected by local conditions. Ballistic transport redistributes the hydrogen atoms in the exosphere, but does not change the global amount of hydrogen in the exosphere. Therefore, it is not surprising to find a limited impact on the simulated brightness. Since the main goal of this study is to focus on the large differences between the simulations and the observations, in the next section we will neglect this effect. A more careful study of the local time variations by few tenths of the brightness as observed by MAVEN (Chaffin et al. 2015) would need to include this effect and such a study is therefore deferred to a future work.

\section{4) Comparisons with observations}

Examples of comparisons between the observed brightness and the simulated brightness at different Martian months are displayed on Fig. 4. The model reasonably reproduces the observations at Ls $=180^{\circ}, 220^{\circ}$ and $340^{\circ}$ but underestimates the brightness at $\mathrm{Ls}=280^{\circ}$ and does not reproduce the shape of the vertical profile. To quantify the difference between the observed and simulated brightness, we derived an optimized scale factor which needed to be applied to the observation to better reproduce the simulated profile by minimizing the function.

$$
\Delta^{2}=\sum_{\text {los }}\left[\log \left(I_{o b s}\right)+\log (A)-\log \left(I_{\text {sim }}\right)\right]^{2}
$$

The minimum of the function $\Delta^{2}$ is found when its derivative $\partial \Delta^{2} / \partial \mathrm{A}=0$ yielding $\mathrm{A}$ as

$$
\log (A)=\frac{1}{n} \sum_{\text {los }}\left[\log \left(I_{\text {sim }}\right)-\log \left(I_{\text {los }}\right)\right]
$$

The rescaled profile is also displayed on Fig.4. For the three observations at $\mathrm{Ls}=180^{\circ}, 220^{\circ}$ and $340^{\circ}$ displayed in Fig. 4 , this scale factor is $0.99,0.79$, and 0.82 respectively, and is in the range of possible uncertainty associated with the retrieved brightness and the solar flux at Mars or the simulation of a monthly average hydrogen corona. For the observation near Ls $=280^{\circ}$, the scale factor is 0.39 , which cannot be explained by the uncertainty in the retrieved brightness by SPICAM-UV.

The difference between the simulated and the observed Lyman- $\alpha$ brightness indicates an important underestimate of the hydrogen density by the model, as was suggested by our first simple comparison of the escape rate derived from SPICAM by Chaffin et al. (2014) at this season (from the same set of observations) and the simulated escape rate for different solar conditions (Chaufray et al. 2015a). The shape of the simulated profile also differs from that of the 
observed profile, confirming that the difference cannot be attributed to the absolute calibration of SPICAM or an

underestimate of the solar flux at Mars and is therefore due to an underestimate of the hydrogen density at this season by our model. Observations performed simultaneously between Ls $=331$ and $345^{\circ}$ by HST during the Martian year 28 also indicate a decrease of the hydrogen corona from this period (Clarke et al. 2014), which is consistent with the SPICAM observations.

Possible explanations of this underestimate of the hydrogen content of the Martian upper atmosphere in our model will be discussed in section 5
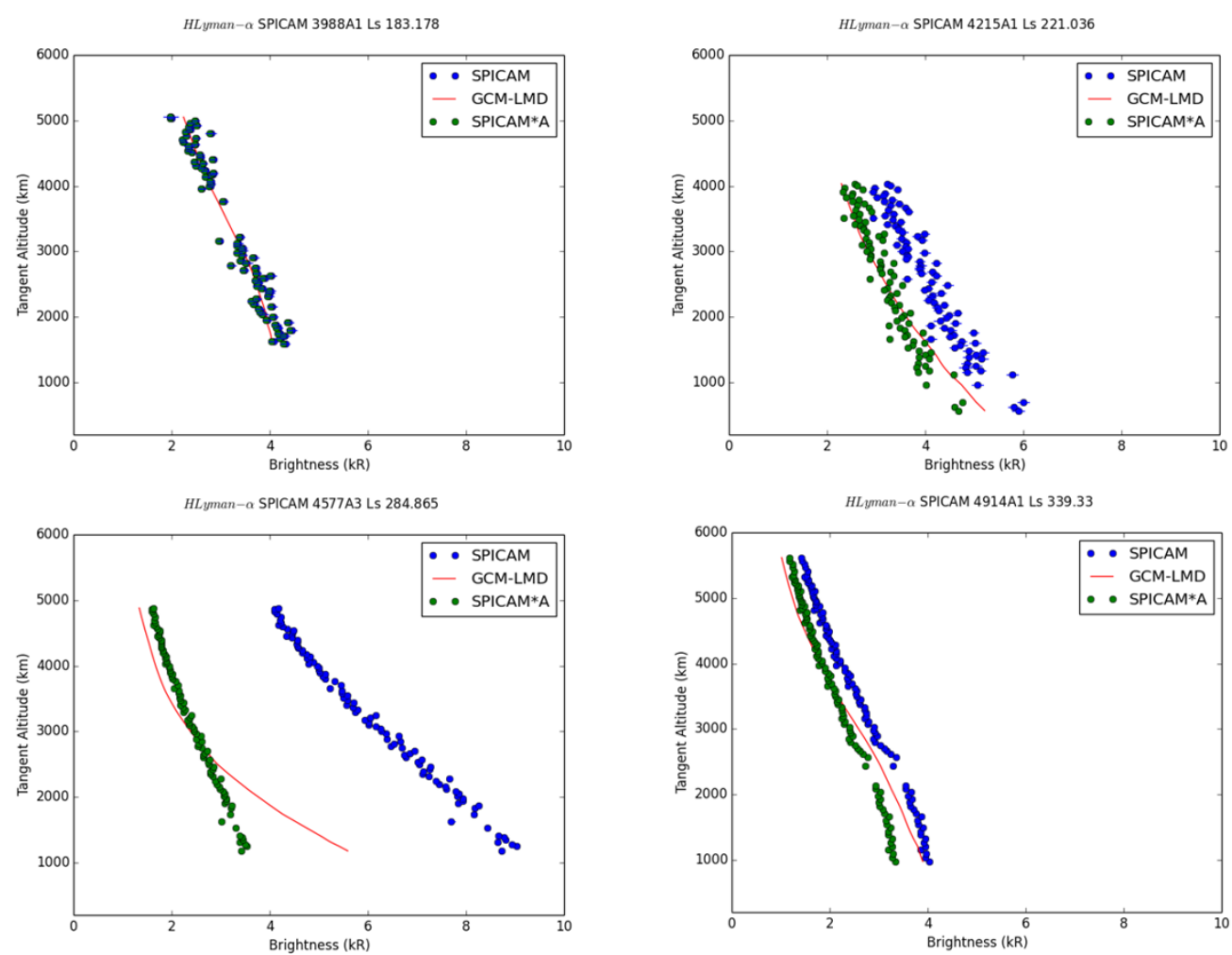

Fig. 4 Examples of comparisons between the Lyman- $\alpha$ brightness profiles observed by SPICAM-UV (blue dots) and the simulated brightness profile (red line) for different solar longitude Ls during the Martian year 28.

Fig. 5 displays the simulated profile corresponding to observation 4577 when the simulated hydrogen density is arbitrarily multiplied by a factor 6 . In that case, the simulated profile is in better agreement with the observation and the simulated shape of the profile is consistent with the observed shape, confirming the difference is likely due to an underestimate of the hydrogen density by the model. 


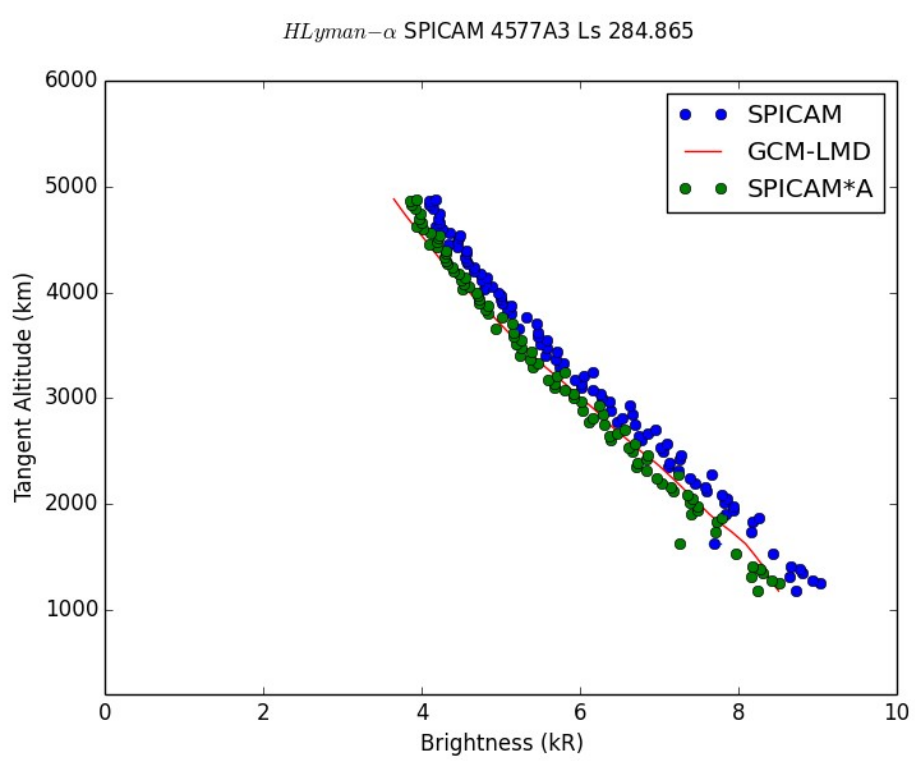

Fig. 5 Simulated Lyman- $\alpha$ brightness profile for orbit \#4577 with a simulated hydrogen density multiplied by 6 w.r.t. the normal model, compared to the SPICAM

Examples of comparisons between the observed brightness and the simulated brightness at different Martian months for Martian year 29 are displayed on Fig. 6. The model reasonably reproduces the observations at Ls near $40^{\circ}, 80^{\circ}$ (the best comparison for the full set of observations), and to a lesser extent, near $145^{\circ}$, but fails to reproduce the observation near $\mathrm{Ls}=255^{\circ}$. The observation at $\mathrm{Ls}=255^{\circ}$ corresponds to a grazing limb observation with a tangent altitude decreasing at the nightside (brightness $<4 \mathrm{kR}$ ) reaching a minimum altitude near $100 \mathrm{~km}$ and increasing on the dayside where the Lyman-alpha brightness is large, $\sim 10 \mathrm{kR}$.

For the Martian year 29, we derive an optimized scale factor $A$ for each observation. The scale factors are $1.08,0.98$, 0.81 and 0.75 for the four profiles displayed on Fig. 5., confirming that the model underestimates the brightness near summer southern solstice. While the factor 0.75 is close to the reasonable value, it corresponds to a large difference in term of hydrogen density, due to the large brightness $(\sim 10 \mathrm{kR})$ and the non-linearity between the density and the brightness for an optically thick emission.

As for the Martian year 28, we performed another simulation with the hydrogen density arbitrarily multiplied by 6 for the observation \#6832. Fig. 7 shows that the agreement is slightly better, as expected, but the brightness is still underestimated. But the remaining difference is less than $1 \mathrm{kR}$ and could be due to the deuterium emission not included in our simulations, this could possibly be important at this season at these low altitudes (Clarke et al. 2017, Chaffin et al. 2018). 

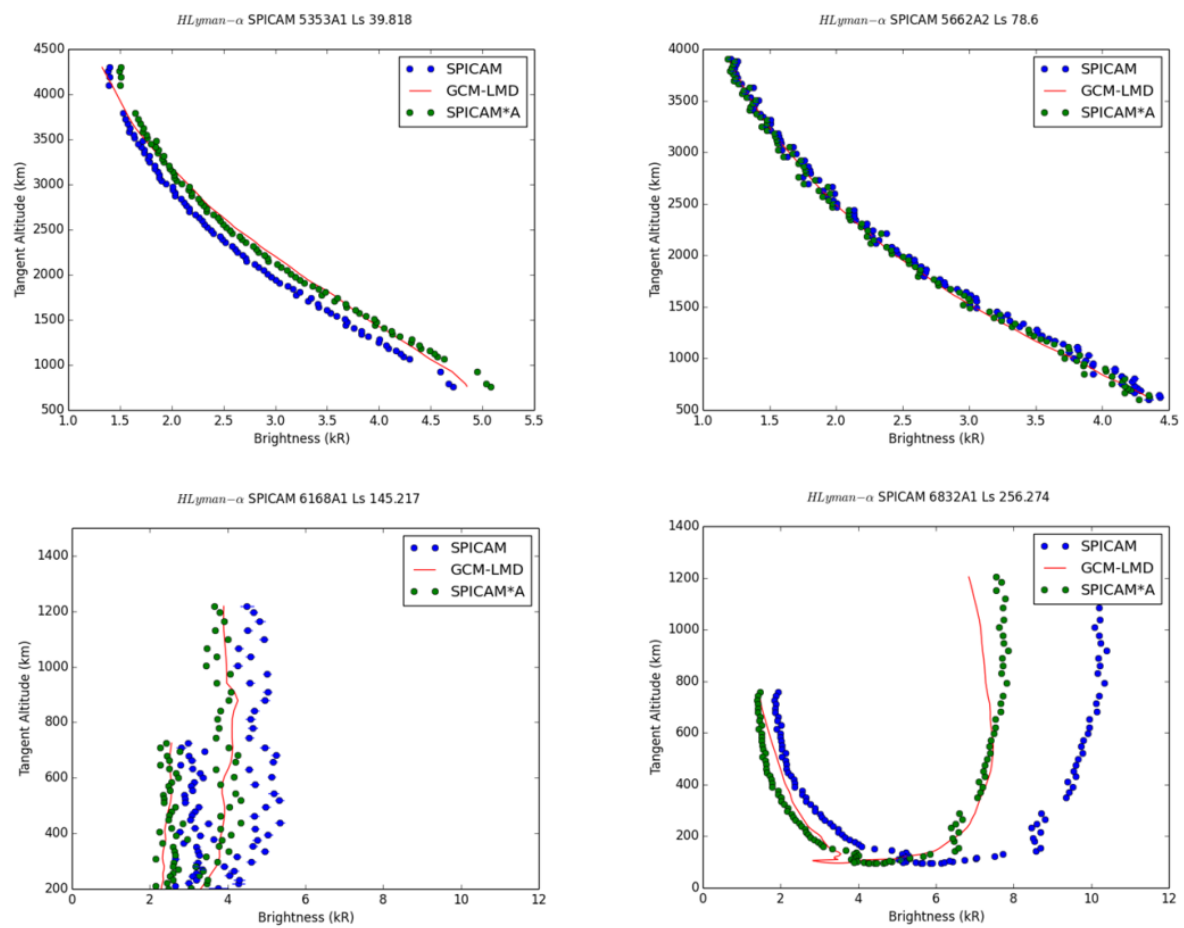

Fig. 6 Examples of comparisons between the Lyman- $\alpha$ brightness profiles observed by SPICAM- UV (blue dots) and the simulated brightness profile (red line) for different solar longitude Ls during the Martian year 29

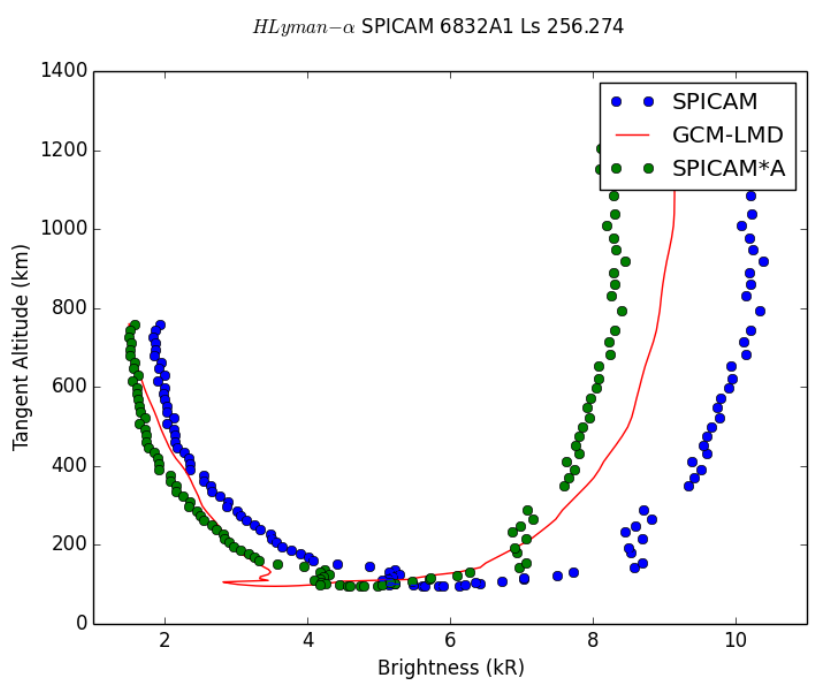

Fig. 7 Simulated Lyman- $\alpha$ brightness profile for orbit \#6832 with a simulated hydrogen density multiplied by 6 w.r.t. the normal model, compared to the SPICAM

For each observation from Martian years 28 and 29, we derive a scale factor and compute the $\chi^{2}$ value for the observed and the rescaled profiles given by:

$$
\chi^{2}=\frac{1}{n} \sum_{l o s} \frac{\left[B^{*} I_{o b s}-I_{s i m}\right]^{2}}{B^{2} \sigma_{o b s}^{2}}
$$

with $\mathrm{B}=1$ for the observed profile and $\mathrm{B}=\mathrm{A}$ for the rescaled profile.

290 Fig.8 displayed the $\chi^{2}$ value versus Ls, including all observations given in Table 1, as well as the scale factor $A$ 

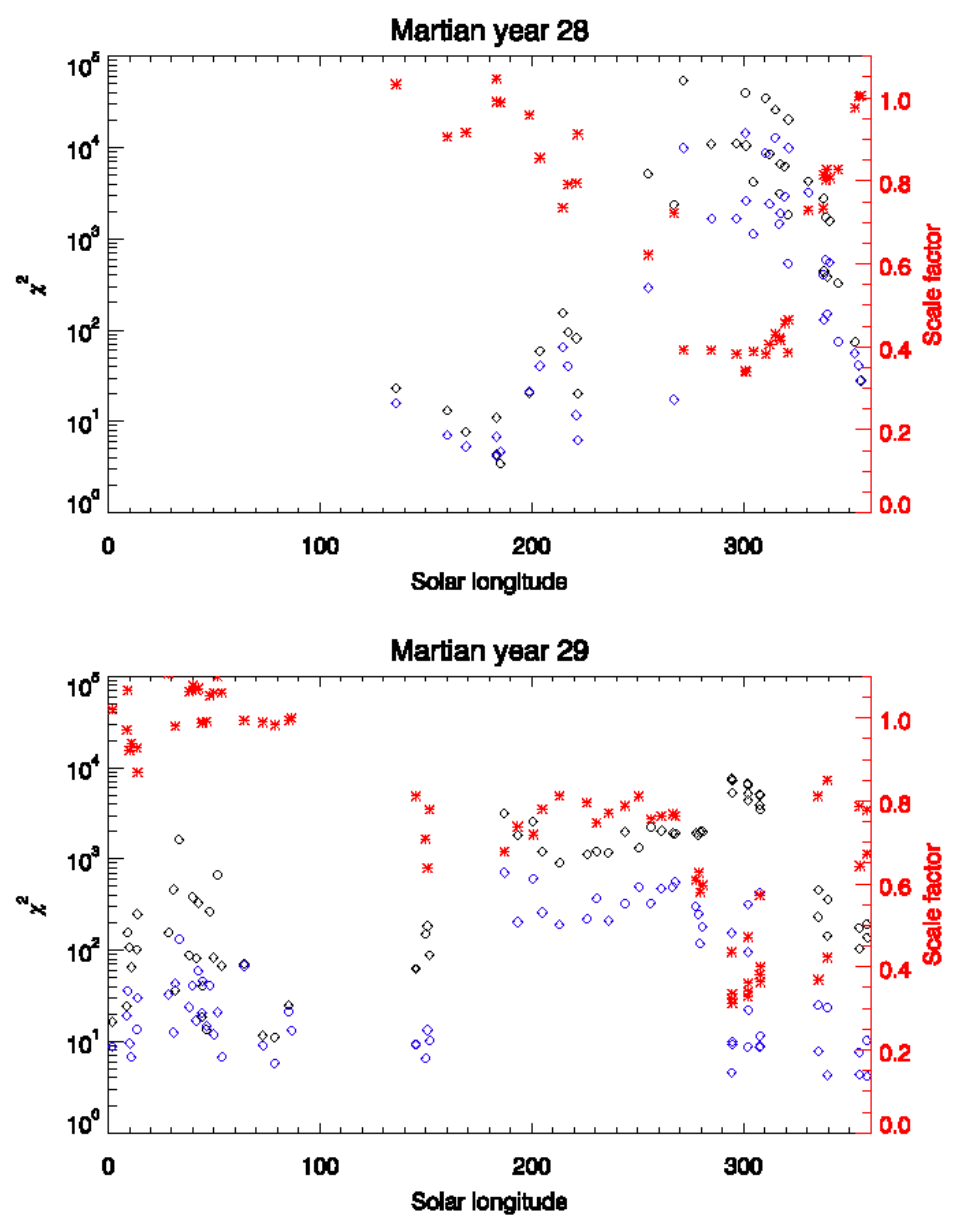

Fig. $8 \chi^{2}$ variations (black diamond, left scale) for the Martian year 28 (top) and Martian year (29), and best scale factor variations (red crosses, right scale), with the associated $\chi^{2}$ values (blue diamonds).

The full set of observations confirm the conclusion derived from the few examples shown in Figs. 4 and 6 . The simulated profiles are in reasonable agreement with the observed profile when $\chi^{2}<100$, and the scale factor is larger than $0.8\left(\mathrm{Ls}<230^{\circ}\right.$ and $\left.\mathrm{Ls}>330^{\circ}\right)$ for Martian year 28, and $\mathrm{Ls}<150^{\circ}$ and $\mathrm{Ls}>340^{\circ}$ for Martian year 29). The scale factor reaches the smallest values $\sim 0.3-0.4$ for $\mathrm{Ls}=250^{\circ}-330^{\circ}$ for both years, meaning the model strongly underestimates the hydrogen density for this season. For Martian year 28, even if the absolute brightness was rescaled, the shape of the profile would not be well reproduced as indicated by the large value of $\chi^{2}$ (see also Fig. 4 bottom left) when the optimized scale factor is included. To reproduce the observed profile, the simulated hydrogen density should be multiplied by $\sim 6$ as shown for orbit 4577 in Fig. 5 .

At Ls near $0^{\circ}$ (observation \#5070), Chaffin et al. (2014) was able to fit the observed profile with an escape flux between $1 \times 10^{7}$ and $1 \times 10^{8} \mathrm{~cm}^{-2} \mathrm{~s}^{-1}$, corresponding to a total escape rate $\sim 1.6 \times 10^{25}$ and $1.6 \times 10^{26} \mathrm{~s}^{-1}$ if we assume a 
uniform escape flux at the exobase in agreement with our simulated escape rate equal to $4 \times 10^{25} \mathrm{~s}^{-1}$ at this season (Fig. 3). The escape flux simulated is not uniform and larger at the dayside than nightside (Chaufray et al. 2015a). If we integrate only the escape flux at the dayside to derive the escape rate, then all the derived escape rate from SPICAM shown in Fig. 3 should be divided by two. In that case, the observations near Ls $=0^{\circ}$ are still in agreement with our simulations. The two other observations detailed in Chaffin et al. (2014) (observation \#4646 and \#4501) are not reproduced by our simulations and the escape rate derived by Chaffin et al. (2014) was between $2 \times 10^{8}$ and $5 \times 10^{9} \mathrm{~cm}^{-}$ ${ }^{2} \mathrm{~s}^{-1}\left(3 \times 10^{26}\right.$ and $\left.8 \times 10^{27} \mathrm{~s}^{-1}\right)$ for observation \#4501 $\left(\mathrm{Ls}=271^{\circ}\right)$ and between $1 \times 10^{8}$ and $2 \times 10^{9} \mathrm{~cm}^{-2} \mathrm{~s}^{-1}\left(1.6 \times 10^{26} \mathrm{~s}^{-1}\right.$ $\left.3.2 \times 10^{27} \mathrm{~s}^{-1}\right)$ for observation $\# 4646\left(\mathrm{Ls}=296^{\circ}\right)$, larger than our simulated escape flux of $\sim 10^{26} \mathrm{~s}^{-1}$ at these periods

(Fig. 2). These high values would be in better agreement with the model escape flux if the simulated hydrogen density was increased by a factor $\sim 6$ as suggested by our simple density rescale.

\section{5) Discussion}

The hydrogen density in the Martian upper atmosphere simulated for Martian years 28 and 29 is in general in reasonable agreement with the SPICAM observations suggesting that the main source processes of atomic hydrogen are included in these simulations during most of the Martian year, except near southern summer solstice $\left(\operatorname{Ls} \sim 270^{\circ}\right)$. At this period, the hydrogen density and the escape rate are likely underestimated by a factor $\sim 6$.

At this period, deuterium Lyman- $\alpha$ could contribute partly to the brightness, especially in the thermosphere / lower exosphere as shown by MAVEN/IUVS (Clarke et al. 2017), for example for orbits 6500s, 6600s and 6700s (Fig. 6 bottom right). However, the deuterium brightness measured by MAVEN/IUVS during Martian year 32 is less than 1 kRayleigh and decreases quickly with altitude, becoming negligible above $300 \mathrm{~km}$. Such a low brightness is insufficient to explain the discrepancy between the model and the observations. Substantial amounts of water vapor at high altitude in the mesosphere has been detected by SPICAM at this season (Maltagliati et al. 2013, Fedorova et al. 2018). The water vapor could be photodissociated and contribute to an increase in the hydrogen density in the Martian thermosphere and exosphere, as well as the hydrogen escape (Chaffin et al. 2017). The MAVEN/NGIMS ionospheric observation above $150 \mathrm{~km}$ near the same season (Martian year 32) suggested a water vapor mixing ratio of only $0.4 \mathrm{ppb}$ at $80 \mathrm{~km}$, in order to avoid the loss of observed $\mathrm{HCO}^{+}$by $\mathrm{H}_{2} \mathrm{O}$ reactions (Fox et al. 2015), but has been recently questionned by another model (Krasnopolsky 2019). In this study, the water vapor density profile was assumed to be close to a diffusion equilibrium profile. Recently, Heavens et al. (2018) suggested that the increase of the hygropause altitude due to dust storms could lead to an increase of the hydrogen density (and escape) in the 
thermosphere, while Shaposhnikov et al. (2019) suggest, using a GCM, that a "pump" mechanism could facilitate upward transport at high latitude during perihelion. Seasonal variations of the altitude of the hygropause at Mars are present in the LMD-GCM simulations (Montmessin et al. 2005). Fig. 9 displays a typical density profile for $\mathrm{H}_{2} \mathrm{H}_{2}$, $\mathrm{H}_{2} \mathrm{O}$, and $\mathrm{CO}_{2}$ and the temperature profile obtained for Martian year 28 at the equator at noon for the month Ls = $120-150$ and $\mathrm{Ls}=270^{\circ}-300^{\circ}$. At $\mathrm{Ls}=120-150^{\circ}$, the water vapor is confined to very low altitudes $(<20 \mathrm{~km})$. The hydrogen density presents a double-peaked profile, one peak in the low atmosphere due to water vapor photodissociation, and another peak in the low thermosphere due to ionospheric reactions (Krasnopolsky 2002). At $\mathrm{Ls}=240-270^{\circ}$, the hygropause is $\sim 50 \mathrm{~km}$, but there are still substantial amounts of water vapor in the mesosphere near $80 \mathrm{~km}$ but not above $120 \mathrm{~km}$. Only one hydrogen density peak is observed, because the peak associated with ionospheric reactions is merged with the hydrogen coming from water photodissociation transported from the lower atmosphere which is $\sim 100$ times larger.
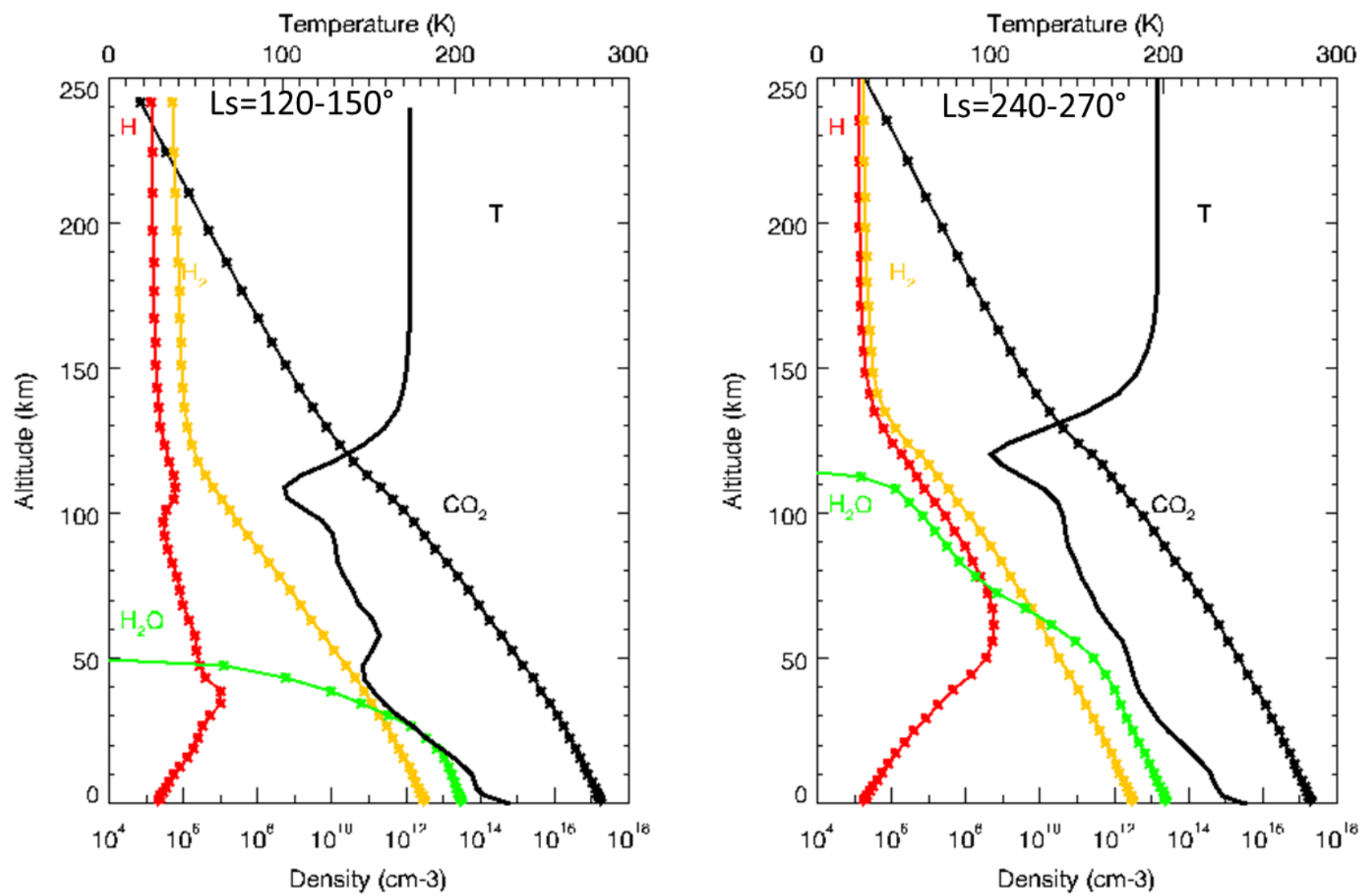

Fig. 9 Vertical density profile of the main hydrogen species and $\mathrm{CO}_{2}$ at noon equator from the surface to the exobase simulated for two different months during the Martian year 28. 
could be the main driver of our simulated escape rate, contrary to our first interpretation (Chaufray et al. 2015a). This would partly explain the difference between our simulations and the model of Krasnopolsky (2002), as suggested by Krasnopolsky (2017). An increase in the water vapor density could explain the current underestimate of the hydrogen escape at Ls $=270^{\circ}-300^{\circ}$, and would be in agreement with the conclusion of Maltagliati et al. (2013), who found that the GCM-LMD underestimates the water vapor in the Martian mesosphere at this season, since the description of the water cycle presented in this study is done with similar assumptions than those used by these authors. This discrepancy was attributed to an underestimation of the strength of the interaction between the water vapor and the dust cycles. The water vapor mixing ratio in the mesosphere depends on the supersaturation of the upper atmosphere, which is not well known. The microphysical processes controlling supersaturation are now included in the LMDGCM (Navarro et al. 2014), but their effects on the water vapor at high altitudes depends on model parameters not well constrained by the observations and therefore were not included in these simulations. A similar comparison with the few SPICAM observations displayed in Fig. 4 of the simulations used to generate the Mars Climate Database 5.2 (for the Martian year 28), including these microphysical processes is displayed in Fig. 10
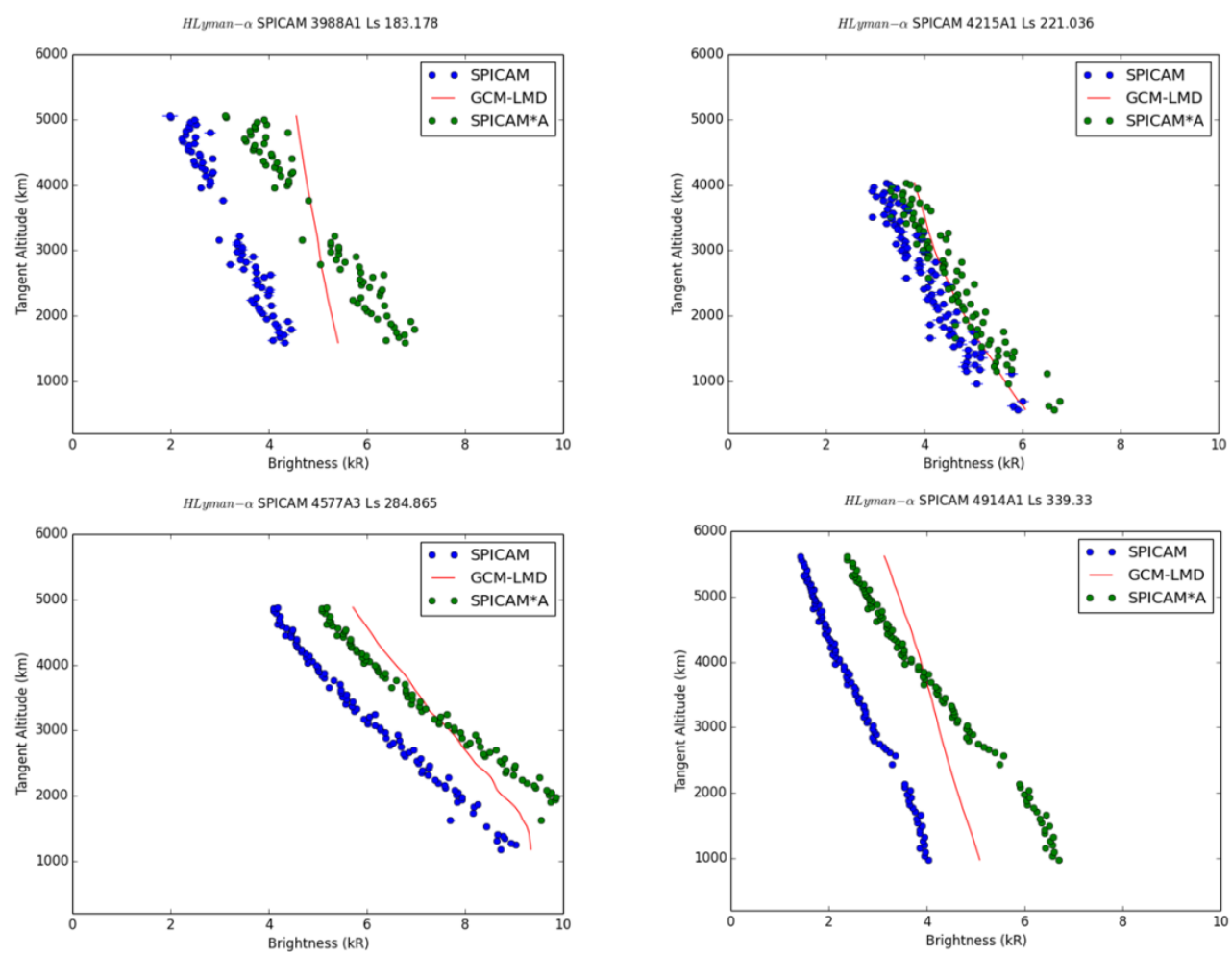

Fig. 10 Same as Fig. 4 but for the simulations used to generate the MCD 5.2, including the microphysical processes controlling the supersaturation.

In these simulations, the Lyman-alpha brightness is overestimated, even at the southern summer. The brightness 
profiles for observations $4215 \mathrm{~A} 1$ is in good agreement, while the simulated shape profiles of the three other observations is very different from the observed shape suggesting that the difference is due to an overestimate of the hydrogen density in the Martian upper altitude. The average hydrogen escape rate for Ls $=270-300^{\circ}$ used to simulate the observation 4501 is $1.1 \times 10^{27} \mathrm{~s}^{-1}$, larger by $\sim 2$ compared with the escape rate estimated in section 4 after a global rescale of the hydrogen density. Although not perfect, the updated LMD-GCM microphysical processes could be sufficient to produce the large amount of hydrogen lacking in our previous simulations. Our simulations could also be consistent with the ionospheric observations by MAVEN/NGIMS, because if the amount of water vapor coming from the lower atmosphere is larger at $120 \mathrm{~km}$, but remains negligible above $150 \mathrm{~km}$, it could prevent the $\mathrm{HCO}^{+}$ destruction while producing $\mathrm{H}_{3} \mathrm{O}^{+}$ions near $120 \mathrm{~km}$ (Fox et al. 2015). A more detailed study of the link between the water vapor and the atomic hydrogen and ionospheric water species will benefit from future observations of the water vapor density in the Martian mesosphere/thermosphere by Exo Mars Trace Gas Orbiter (Lopez-Valverde et al. 2018), especially during the recent global dust storm in June 2018. Comparisons of other Martian years like Martian year 32 where an increase of water vapor was also observed by SPICAM (Fedorova et al. 2018), and using the large Lyman- $\alpha$ dataset of MAVEN/IUVS (Chaffin et al. 2015, 2018) will be also useful to estimate the year to year variability possibly due to dust events and the variability associated with the solar activity in order to extrapolate past conditions with larger EUV solar flux and orbit parameters. Space weather events also increase the atmospheric escape rates (Jakosky et al.. 2015). But they mostly increase the heavy ions escape rates as reported in Jakosky et al. (2015) during the interplanetary coronal mass ejection in March 2015. Solar flares could heat the Martian upper atmosphere and increase the escape rate during short time periods (Mayyasi et al. 2018).

Considering the discrepancy of our model and the SPICAM observations, we estimated the hydrogen escape rate during these two Martian years to vary between $\sim 10^{25}$ to $6 \times 10^{26} \mathrm{~s}^{-1}$. This range is in good agreement the with the seasonal variations $\left(3 \times 10^{25} \mathrm{~s}^{-1}\right.$ near aphelion and $4 \times 10^{26} \mathrm{~s}^{-1}$ near perihelion) reported from pick-up protons for the Martian years 32-33 by Rahmati et al. (2018), and from Lyman- $\alpha$ emission by Chaffin et al. (2018) (estimated $~ 5$ $14 \times 10^{26} \mathrm{~s}^{-1}$ at Ls $=250^{\circ}$ for MY 32 and $1-4 \times 10^{26} \mathrm{~s}^{-1}$ at Ls $=200^{\circ}$ for MY 33). It is slightly lower than the escape rate estimated from the hydrogen column upstream of the bow shock by Halekas (2017); considering $\mathrm{T}=200 \mathrm{~K}$ near aphelion, and $\mathrm{T}=300 \mathrm{~K}$ near perihelion, and assuming an uniform escape rate, the escape flux derived from Fig. 5 of Halekas (2017) leads to an escape rate variation from $\sim 3 \times 10^{25}$ to $\sim 3 \times 10^{27} \mathrm{~s}^{-1}$. If we express this escape flux into a water Global Equivalent Layer (GEL) $d$ using 
$d(m / G y r)=\frac{\Phi_{H}}{2} \frac{m_{H_{2} \mathrm{O}}}{\rho_{\mathrm{H}_{2} \mathrm{O}} S} T$,

where $\Phi_{H} / 2$ is the water loss rates, $\mathrm{m}_{\mathrm{H} 2 \mathrm{O}}$ the mass of a water molecule, $\rho_{\mathrm{H} 2 \mathrm{O}}$ water mass density, $S$ the Martian surface and $T$ the number of seconds in $1 \mathrm{Gyr}\left(\sim 3.2 \times 10^{16} \mathrm{~s} / \mathrm{Gyr}\right)$, we find a loss between $\sim 33$ to $2000 \mathrm{~mm}$ per billion years and therefore no more than $10 \mathrm{~m}$ GEL during the last 4 billion years, which is small compared to the total water content measured in the reservoirs at the surface and the subsurface $(\sim 30 \mathrm{~m})$, and even less compared to the estimated amount of water needed to form the valley networks or outflow channels (estimated to few 100s m GEL with large uncertainties, Lasue et al. 2013). The differences could be either due to a more important hydrogen escape rate in the past or to a larger reservoir of water (ice or liquid) in the subsurface inaccessible or not yet detected by current radar (Orosei et al. 2015, 2018).

\section{6) Conclusion}

We simulated the atomic hydrogen content in the upper atmosphere of Mars for Martian years 28 and 29. These two years correspond to a period of numerous hydrogen coronal observations by SPICAM on Mars Express. The comparison between our simulated brightness and SPICAM observations shows a reasonable agreement with the observations for $\mathrm{Ls}<200^{\circ}$ and $\mathrm{Ls}>330^{\circ}$ for Martian year 28, for $\mathrm{Ls}<150^{\circ}$ and Ls $>340^{\circ}$ for Martian year 29, while the simulated brightness is strongly underestimated for $230<\mathrm{Ls}<330^{\circ}$ for Martian year 28 and $270^{\circ}<\mathrm{Ls}<340^{\circ}$ for Martian year 29 (therefore, around and after the southern solstice equinox at $\mathrm{Ls}=270^{\circ}$ and the perihelion crossing at $\mathrm{Ls}=251^{\circ}$ ). This underestimate corresponds to a model underestimate of the global hydrogen density (and the Jeans escape rate) by a factor $\sim 6$. Therefore, our study confirms that the seasonal variations of the hydrogen escape at Mars could be of almost two orders of magnitude, as suggested by Chaffin et al. (2014). It also confirms that this variation is a seasonal variation which may be expected every Martian year, as observed from plasma measurements by Mars Express (Yamauchi et al. 2015) and more recently by MAVEN for the Martian year 32, and 33 (e.g., Clarke et al. 2017, Halekas 2017, Rahmati et al. 2018, Chaffin et al. 2018). The discrepancy between our simulations and the observations is attributed to an LMD-GCM underestimate of the amount of water vapor transported to high altitudes. Accurate observations of the water vapor density in the Martian upper atmosphere by TGO (Vandaele et al. 2019) or MAVEN/NGIMS could help to better the understanding of the details of this transport.

\section{Acknowledgements}


This project was partially funded by the Programme National de Planetologie and Programme National Soleil Terre and by the Centre National d'Etudes Spatiales. This work has been partially funded by the European Union Horizon 2020 Programme (H2020 Compet -08-2014) under grant agreement UPWARDS-633127. We would like to thank G. Lacombe and L. Baggio for their support on the calibration. We thank Allyson Leffler for her careful review of grammatical aspects of this paper. The SPICAM/MEX data used in this study are available through the ESA Planetary Science Archive (PSA, https://archives.esac.esa.int/psa/). The monthly average 3D simulated hydrogen exosphere can be found on : https://owncloud.latmos.ipsl.fr/index.php/s/AdUgv9PdipA2rim

\section{Appendix : List of SPICAM-UV observations used in this study.}

The list of the SPICAM observations used for the Martian year 28 and 29, and some geometric parameters are given in Table 1 and Table 2 respectively

\begin{tabular}{|c|c|c|c|c|c|c|c|c|}
\hline $\begin{array}{l}\text { Orbit and } \\
\text { Sequence }\end{array}$ & Date & Ls & $\begin{array}{l}\text { Altitude } \\
\text { range }(\mathrm{km})\end{array}$ & $\begin{array}{l}\text { SZA } \\
\text { range }\end{array}$ & $\begin{array}{l}\text { Latitude } \\
\text { range }\end{array}$ & $\begin{array}{l}\text { Slit/N } \\
\text { o slit }\end{array}$ & Binning $/ Y_{0}$ & $\begin{array}{l}\text { Band } \\
\text { used }\end{array}$ \\
\hline $3668 \mathrm{~A} 1$ & 15 Nov 2006 & 135.4 & $600-5600$ & $56^{\circ}$ & $32 \mathrm{~S}$ & Slit & $32 / 22$ & 5 \\
\hline $3838 \mathrm{~A} 2$ & 02 Jan 2007 & 159.8 & $500-5500$ & $57^{\circ}$ & $7 \mathrm{~N}$ & Slit & $32 / 22$ & 5 \\
\hline 3896A1 & 18 Jan 2007 & 168.4 & $600-5600$ & $66^{\circ}$ & $5 \mathrm{~N}$ & Slit & $32 / 22$ & 5 \\
\hline 3988A1 & 13 Feb 2007 & 182.9 & $600-5000$ & $79^{\circ}$ & $23 \mathrm{~S}$ & Slit & $32 / 22$ & 5 \\
\hline 3989A1 & $13 \mathrm{Feb} 2007$ & 182.9 & $500-4500$ & $79^{\circ}$ & $37 \mathrm{~S}$ & Slit & $32 / 22$ & 5 \\
\hline $4001 \mathrm{~A} 1$ & 17 Feb 2007 & 185.1 & $500-4800$ & $80^{\circ}$ & $26 \mathrm{~S}$ & Slit & $32 / 22$ & 5 \\
\hline $4085 \mathrm{~A} 1$ & 12 Mar 2007 & 198.6 & $1000-4300$ & $39-67$ & $1 \mathrm{~S}-18 \mathrm{~S}$ & Slit & $32 / 22$ & 5 \\
\hline 4114A1 & 20 Mar 2007 & 203.3 & $900-4600$ & $40-73$ & $12 \mathrm{~S}-32 \mathrm{~S}$ & Slit & $32 / 22$ & 5 \\
\hline 4178A1 & 07 Apr 2007 & 214.3 & $800-4500$ & $50-86$ & $3 S-28 S$ & Slit & $32 / 22$ & 5 \\
\hline $4192 \mathrm{~A} 1$ & 11 Apr 2007 & 216.7 & $500-4100$ & $50-88$ & $4 \mathrm{~N}-24 \mathrm{~S}$ & Slit & $32 / 22$ & 5 \\
\hline $4215 \mathrm{~A} 1$ & 17 Apr 2007 & 220.4 & $500-4100$ & $55-93$ & $7 \mathrm{~N}-22 \mathrm{~S}$ & Slit & $32 / 22$ & 5 \\
\hline 4219A1 & 18 Apr 2007 & 221.0 & $400-3600$ & $57-92$ & $19 N-13 S$ & Slit & $32 / 22$ & 5 \\
\hline 4407A4 & 10 Jun 2007 & 254.4 & $500-4000$ & $10-80$ & $14 \mathrm{~S}-54 \mathrm{~N}$ & Slit & $32 / 118$ & 4 \\
\hline $4475 \mathrm{~A} 1$ & 29 Jun 2007 & 266.5 & $400-4200$ & $36-86$ & $4 S-58 N$ & Slit & $32 / 118$ & 4 \\
\hline $4501 \mathrm{~A} 1$ & 07 Jul 2007 & 271.5 & $900-4300$ & $14-67$ & $14 \mathrm{~S}-44 \mathrm{~N}$ & No Slit & $32 / 118$ & 1 \\
\hline
\end{tabular}




\begin{tabular}{|c|c|c|c|c|c|c|c|c|}
\hline $4577 \mathrm{~A} 3$ & $28 \mathrm{Jul} 2007$ & 284.6 & $1100-4800$ & $8-60$ & $16 \mathrm{~S}-34 \mathrm{~N}$ & Slit & $32 / 118$ & 4 \\
\hline 4646A1 & 16 Aug 2007 & 296.2 & $1300-5600$ & 58 & $28 \mathrm{~N}$ & Slit & $32 / 118$ & 4 \\
\hline $4671 \mathrm{~A} 2$ & 23 Aug 2007 & 300.4 & $1100-5100$ & $47^{\circ}$ & $10 \mathrm{~N}$ & No Slit & $32 / 118$ & 1 \\
\hline $4673 \mathrm{~A} 2$ & 24 Aug 2007 & 301.0 & $1300-5500$ & $51^{\circ}$ & $17 \mathrm{~N}$ & Slit & $32 / 118$ & 4 \\
\hline $4693 \mathrm{~A} 2$ & 29 Aug 2007 & 304.0 & $300-10000$ & $36-96$ & $5 \mathrm{~N}-76 \mathrm{~N}$ & Slit & $32 / 118$ & 4 \\
\hline 4729A1 & 08 Sep 2007 & 309.8 & $1100-3500$ & $45^{\circ}$ & $23 \mathrm{~S}$ & No slit & $32 / 118$ & 1 \\
\hline 4740A1 & 11 Sep 2007 & 311.6 & $1100-3600$ & $47^{\circ}$ & $25 \mathrm{~S}$ & Slit & $32 / 118$ & 4 \\
\hline $4757 \mathrm{~A} 2$ & 16 Sep 2007 & 314.5 & $700-3600$ & $50^{\circ}$ & $28 \mathrm{~S}$ & No slit & $32 / 118$ & 1 \\
\hline 4769A2 & 20 Sep 2007 & 316.8 & $300-10000$ & $19-85$ & $1 S-63 N$ & Slit & $32 / 118$ & 4 \\
\hline $4772 \mathrm{~A} 2$ & $20 \operatorname{Sep} 2007$ & 316.8 & $700-3600$ & 53 & $31 \mathrm{~S}$ & Slit & $32 / 118$ & 4 \\
\hline $4785 \mathrm{~A} 1$ & 24 Sep 2007 & 319.1 & $700-3600$ & 56 & $33 \mathrm{~S}$ & Slit & $32 / 118$ & 4 \\
\hline 4794A2 & 27 Sep 2007 & 320.8 & $800-10000$ & $19-90$ & $2 \mathrm{~N}-60 \mathrm{~N}$ & Slit & $32 / 118$ & 4 \\
\hline 4796A1 & 27 Sep 2007 & 320.8 & $700-3600$ & 57 & $34 \mathrm{~S}$ & No Slit & $32 / 118$ & 1 \\
\hline 4856A1 & 14 Oct 2007 & 330.2 & $700-3600$ & 69 & $45 \mathrm{~S}$ & No Slit & $32 / 118$ & 1 \\
\hline 4903A1 & 27 Oct 2007 & 337.3 & $1400-5500$ & 76 & $23 \mathrm{~S}$ & No Slit & $32 / 118$ & 1 \\
\hline 4905A1 & 28 Oct 2007 & 337.8 & $700-5600$ & 77 & $10 \mathrm{~S}$ & Slit & $32 / 118$ & 4 \\
\hline 4910A1 & 29 Oct 2007 & 338.4 & $1000-5600$ & 77 & $17 \mathrm{~S}$ & No Slit & $32 / 118$ & 1 \\
\hline 4914A1 & 30 Oct 2007 & 338.9 & $1000-5600$ & 78 & $18 \mathrm{~S}$ & Slit & $32 / 118$ & 4 \\
\hline 4923A1 & 02 Nov 2007 & 340.5 & $700-5000$ & 79 & $34 \mathrm{~S}$ & No Slit & $32 / 118$ & 1 \\
\hline 4950A1 & 09 Nov 2007 & 344.2 & $700-5000$ & 84 & $39 \mathrm{~S}$ & Slit & $32 / 118$ & 4 \\
\hline $5005 \mathrm{~A} 1 \mathrm{a}$ & 25 Nov 2007 & 352.5 & $300-3300$ & $32-56$ & $24 \mathrm{~S}-9 \mathrm{~N}$ & Slit & $32 / 118$ & 4 \\
\hline $5016 \mathrm{~A} 1$ & 28 Nov 2007 & 354.0 & $600-3600$ & $35-59$ & $14 \mathrm{~S}-14 \mathrm{~N}$ & Slit & $32 / 118$ & 4 \\
\hline $5024 \mathrm{~A} 1$ & 30 Nov 2007 & 355.1 & $500-3700$ & $34-61$ & $17 \mathrm{~N}-29 \mathrm{~N}$ & Slit & $32 / 118$ & 4 \\
\hline $5052 \mathrm{~A} 1$ & 08 Dec 2007 & 359.1 & $500-3900$ & $36-66$ & $18 \mathrm{~N}-31 \mathrm{~N}$ & Slit & $32 / 118$ & 4 \\
\hline
\end{tabular}

Table 1 : List of SPICAM-UV observations used to study the Martian hydrogen corona during the Martian year 28. 439 The orbit number and sequence number of the observations are given in column 1. Column 3 gives the approximate solar longitude Ls at the observation day. Columns 4 and 5 refer to the tangent point of the line of sight.

\begin{tabular}{|c|c|c|c|c|c|c|c|c|}
\hline $\begin{array}{l}\text { Orbit and } \\
\text { Sequence }\end{array}$ & Date & Ls & Altitude range & SZA range & Latitude range & $\begin{array}{l}\text { Slit/No } \\
\text { slit }\end{array}$ & $\begin{array}{l}\text { Binning/Y } \\
0\end{array}$ & $\begin{array}{l}\text { Band } \\
\text { used }\end{array}$ \\
\hline 5070A1 & 13 Dec 2007 & 1.6 & $800-4300$ & $41-70$ & $15 \mathrm{~N}-29 \mathrm{~N}$ & Slit & $32 / 118$ & 4 \\
\hline 5097A1 & 21 Dec 2007 & 5.5 & $900-10,000$ & $75-107$ & $0-60 \mathrm{~N}$ & Slit & $32 / 118$ & 4 \\
\hline 5119A1 & 27 Dec 2007 & 8.5 & $1000-4800$ & $50-80$ & $16 \mathrm{~N}-32 \mathrm{~N}$ & Slit & $32 / 118$ & 4 \\
\hline
\end{tabular}




\begin{tabular}{|c|c|c|c|c|c|c|c|c|}
\hline 5122A1 & 28 Dec 2007 & 8.9 & $1000-4500$ & $50-80$ & $7 \mathrm{~N}-25 \mathrm{~N}$ & No Slit & $32 / 118$ & 1 \\
\hline 5129A1 & 30 Dec 2007 & 9.9 & $900-4400$ & $50-80$ & $4 N-22 N$ & Slit & $32 / 118$ & 4 \\
\hline 5135A1 & 31 Dec 2007 & 10.4 & $900-4600$ & $50-82$ & $14 \mathrm{~N}-33 \mathrm{~N}$ & Slit & $32 / 118$ & 4 \\
\hline 5154A1 & 06 Jan 2008 & 13.3 & $950-4500$ & $55-85$ & $0-20 \mathrm{~N}$ & Slit & $32 / 118$ & 4 \\
\hline 5156A1 & 06 Jan 2008 & 13.3 & $1100-4900$ & $56-87$ & $13 \mathrm{~N}-32 \mathrm{~N}$ & Slit & $32 / 118$ & 4 \\
\hline 5266A1 & 07 Feb 2008 & 28.3 & $450-4300$ & $40-99$ & $23 \mathrm{~S}-85 \mathrm{~S}$ & Slit & $32 / 118$ & 4 \\
\hline 5283A1 & 12 Feb 2008 & 30.6 & $700-4500$ & $48-99$ & $34 \mathrm{~S}-71 \mathrm{~S}$ & No Slit & $32 / 118$ & 1 \\
\hline 5290A2 & $14 \mathrm{Feb} 2008$ & 31.5 & $700-4500$ & $48-99$ & $34 \mathrm{~S}-72 \mathrm{~S}$ & Slit & $32 / 118$ & 4 \\
\hline 5304A1 & 18 Feb 2008 & 33.3 & $600-4400$ & $40-97$ & $22 S-83 S$ & No Slit & $32 / 118$ & 1 \\
\hline 5341A1 & $28 \mathrm{Feb} 2008$ & 37.9 & $600-4400$ & $35-95$ & $19 \mathrm{~S}-78 \mathrm{~S}$ & Slit & $32 / 118$ & 4 \\
\hline $25353 \mathrm{~A} 1$ & 03 Mar 2008 & 39.7 & $500-4300$ & $31-92$ & $14 \mathrm{~S}-76 \mathrm{~S}$ & No Slit & $32 / 118$ & 1 \\
\hline 5367A1 & 07 Mar 2008 & 41.5 & $500-4400$ & $33-93$ & $16 \mathrm{~S}-75 \mathrm{~S}$ & Slit & $32 / 118$ & 4 \\
\hline 2 5374A1 & 09 Mar 2008 & 42.4 & $500-4300$ & $30-92$ & $12 \mathrm{~S}-73 \mathrm{~S}$ & No Slit & $32 / 118$ & 1 \\
\hline 5388A1 & 13 Mar 2008 & 44.2 & $600-4000$ & $52-101$ & $29 \mathrm{~S}-59 \mathrm{~S}$ & Slit & $32 / 118$ & 4 \\
\hline 5390A1 & 13 Mar 2008 & 44.2 & $600-4000$ & $52-101$ & $28 \mathrm{~S}-59 \mathrm{~S}$ & No Slit & $32 / 118$ & 1 \\
\hline 5404A1 & 17 Mar 2008 & 45.9 & $600-4000$ & $50-100$ & $26 \mathrm{~S}-62 \mathrm{~S}$ & Slit & $32 / 118$ & 4 \\
\hline 5416A1 & 21 Mar 2008 & 47.7 & $600-4100$ & $47-98$ & $22 \mathrm{~S}-64 \mathrm{~S}$ & No Slit & $32 / 118$ & 1 \\
\hline 5432A1 & 25 Mar 2008 & 49.5 & $600-4100$ & $46-98$ & $21 \mathrm{~S}-65 \mathrm{~S}$ & Slit & $32 / 118$ & 4 \\
\hline 5446A1 & 29 Mar 2008 & 51.3 & $600-4200$ & $43-95$ & $17 S-66 S$ & No Slit & $32 / 118$ & 1 \\
\hline 5462A1 & 03 Apr 2008 & 53.5 & $600-4000$ & $51-100$ & $21 \mathrm{~S}-63 \mathrm{~S}$ & Slit & $32 / 118$ & 4 \\
\hline 5546A1 & 27 Apr 2008 & 64.0 & $400-3600$ & $57-100$ & $12 \mathrm{~S}-59 \mathrm{~S}$ & Slit & $32 / 118$ & 4 \\
\hline $5617 \mathrm{~A} 2$ & 17 May 2008 & 72.7 & $500-4000$ & $40-77$ & $14 \mathrm{~N}-43 \mathrm{~S}$ & Slit & $32 / 118$ & 4 \\
\hline $5662 \mathrm{~A} 2$ & 30 May 2008 & 78.4 & $400-3900$ & $35-70$ & $17 \mathrm{~N}-39 \mathrm{~S}$ & Slit & $32 / 118$ & 4 \\
\hline $5715 \mathrm{~A} 2$ & 14 Jun 2008 & 85.0 & $800-3400$ & $60-91$ & $12 S-46 S$ & No Slit & $32 / 118$ & 1 \\
\hline 5726A2 & 17 Jun 2008 & 86.3 & $900-3300$ & $58-89$ & $11 \mathrm{~S}-45 \mathrm{~S}$ & No Slit & $32 / 118$ & 1 \\
\hline $5759 \mathrm{~A} 2$ & 26 Jun 2008 & 90.7 & $300-1900$ & $30-70$ & $23 \mathrm{~N}-31 \mathrm{~S}$ & Slit & $32 / 118$ & 4 \\
\hline 6168A1* & 21 Oct 2008 & 144.9 & $730-200-1200$ & $105-48-49$ & $52 \mathrm{~S}-61 \mathrm{~N}$ & Slit & $32 / 23$ & 5 \\
\hline $6200 \mathrm{~A} 1 *$ & 30 Oct 2008 & 149.4 & $900-200-1300$ & $101-51-55$ & $64 \mathrm{~S}-61 \mathrm{~N}$ & Slit & $32 / 23$ & 5 \\
\hline $6206 \mathrm{~A} 1 *$ & 01 Nov 2008 & 150.5 & $1200-200-1300$ & $95-59-64$ & $73 \mathrm{~S}-60 \mathrm{~N}$ & Slit & $32 / 23$ & 5 \\
\hline $6213 \mathrm{~A} 1 *$ & 03 Nov 2008 & 151.5 & $900-200-1300$ & $100-50-55$ & $66 \mathrm{~S}-60 \mathrm{~N}$ & Slit & $32 / 23$ & 5 \\
\hline 6440A1 & 07 Jan 2009 & 187.0 & $900-110-1200$ & $87-72-78$ & $73 \mathrm{~S}-23 \mathrm{~N}$ & Slit & $32 / 118$ & 4 \\
\hline 6478A1* & 18 Jan 2009 & 193.3 & $1200-200-600$ & $100-46-49$ & $23 \mathrm{~S}-61 \mathrm{~S}-16 \mathrm{~S}$ & Slit & $32 / 118$ & 4 \\
\hline
\end{tabular}




\begin{tabular}{|c|c|c|c|c|c|c|c|c|}
\hline $6521 \mathrm{~A} 1$ & 30 Jan 2009 & 200.6 & $1300-100-1200$ & $130-35$ & $40 \mathrm{~S}-73 \mathrm{~S}-2 \mathrm{~S}$ & Slit & $32 / 118$ & 4 \\
\hline 6546A1* & 06 Feb 2009 & 204.9 & $1300-200-1100$ & $120-28$ & 27S-65S-13S & Slit & $32 / 118$ & 4 \\
\hline 6593A1* & 20 Feb 2009 & 213.1 & $1100-200-500$ & $120-44$ & $3 \mathrm{~S}-72 \mathrm{~S}-42 \mathrm{~S}$ & Slit & $32 / 118$ & 4 \\
\hline 6666A1 & 13 Mar 2009 & 226.1 & $1300-100-1300$ & $140-12$ & $19 \mathrm{~S}-74 \mathrm{~S}-23 \mathrm{~S}$ & Slit & $32 / 118$ & 4 \\
\hline 6691A3* & 20 Mar 2009 & 230.4 & $1300-200-1300$ & $140-13$ & $14 \mathrm{~S}-66 \mathrm{~S}-26 \mathrm{~S}$ & Slit & $32 / 118$ & 4 \\
\hline $6722 \mathrm{~A} 1 *$ & 29 Mar 2009 & 236.2 & $1300-200-1300$ & $150-20$ & 9S-67S-31S & Slit & $32 / 118$ & 4 \\
\hline 6765A1 & 10 Apr 2009 & 244.1 & $900-100-1200$ & $135-10$ & $18 \mathrm{~S}-70 \mathrm{~S}-24 \mathrm{~S}$ & Slit & $32 / 118$ & 4 \\
\hline 6801A1 & 20 Apr 2009 & 250.0 & $1300-100-1200$ & $140-35$ & $7 \mathrm{~N}-77 \mathrm{~S}-27 \mathrm{~S}$ & Slit & $32 / 118$ & 4 \\
\hline 6832A1 & 29 Apr 2009 & 256.3 & $800-100-1200$ & $140-10$ & 9S-66S-32S & Slit & $32 / 118$ & 4 \\
\hline 6859A1 & 07 May 2009 & 261.2 & $900-90-1200$ & $140-15$ & $2 \mathrm{~S}-66 \mathrm{~S}-39 \mathrm{~S}$ & Slit & $32 / 118$ & 4 \\
\hline 6888A1 & 15 Мay 2009 & 266.5 & $900-90-1200$ & $140-20$ & $3 \mathrm{~N}-65 \mathrm{~S}-43 \mathrm{~S}$ & Slit & $32 / 118$ & 4 \\
\hline 6895A1 & 17 May 2009 & 267.7 & $900-90-1200$ & $140-20$ & $4 \mathrm{~N}-65 \mathrm{~S}-44 \mathrm{~S}$ & Slit & $32 / 118$ & 4 \\
\hline 6949A1 & 02 Jun 2009 & 277.5 & $1300-10-900$ & $150-65$ & $42 \mathrm{~N}-87 \mathrm{~S}$ & Slit & $32 / 118$ & 4 \\
\hline 6956A2 & 04 Jun 2009 & 278.4 & $1300-10-900$ & $150-65$ & $43 N-87 S$ & Slit & $32 / 118$ & 4 \\
\hline 6960A1 & 05 Jun 2009 & 279. & $1300-10-800$ & $144-74$ & $41 \mathrm{~N}-81 \mathrm{~S}$ & Slit & $32 / 118$ & 4 \\
\hline 6967A1 & 07 Jun 2009 & 280.3 & $1300-10-800$ & $145-75$ & $42 \mathrm{~N}-80 \mathrm{~S}$ & Slit & $32 / 118$ & 4 \\
\hline 7045A2\&3 & 29 Jun 2009 & 294. & $3300-8500-7700$ & $64-84$ & $19 S-14 S$ & Slit & $32 / 118$ & 4 \\
\hline $7046 \mathrm{~A} 1 \& 2^{+}$ & 30 Jun 2009 & & & & & & & \\
\hline 7089A3\&4 & 12 Jul 2009 & 301.6 & $2900-9200-8800$ & $70-89$ & $20 \mathrm{~S}-14 \mathrm{~S}$ & Slit & $32 / 118$ & 4 \\
\hline 7090A1\&2 & 12 Jul 2009 & & & & & & & \\
\hline $7122 \mathrm{~A} 2 \& 3$ & 22 Jul 2009 & 307.5 & $2400-9600-9300$ & $73-94$ & $21 \mathrm{~S}-15 \mathrm{~S}$ & Slit & $32 / 118$ & 4 \\
\hline 7123A1\&2 & 22 Jul 2009 & & & & & & & \\
\hline 7292A1 & 08 Sep 2009 & 334.6 & $2500-4000$ & $53-58$ & $58 \mathrm{~S}-40 \mathrm{~S}$ & Slit & $32 / 118$ & 4 \\
\hline 7293A1 & 09 Sep 2009 & 335.2 & $2800-4300$ & $54-59$ & $57 \mathrm{~S}-39 \mathrm{~S}$ & Slit & $32 / 118$ & 4 \\
\hline$\overline{7320 A 2}$ & 17 Sep 2009 & 339.5 & $2100-4100$ & $56-63$ & $67 \mathrm{~S}-52 \mathrm{~S}$ & Slit & $32 / 118$ & 4 \\
\hline 7321A2 & 17 Sep 2009 & 339.5 & $2400-4300$ & $57-63$ & $67 \mathrm{~S}-52 \mathrm{~S}$ & Slit & $32 / 118$ & 4 \\
\hline $7421 \mathrm{~A} 1$ & 16 Oct 2009 & 354.6 & $4200-5600$ & 78 & $74 \mathrm{~S}$ & Slit & $32 / 118$ & 4 \\
\hline $7423 \mathrm{~A} 1$ & 16 Oct 2009 & 354.6 & $200-3000$ & 76 & $76 \mathrm{~S}$ & Slit & $32 / 118$ & 4 \\
\hline 7446A1 & 23 Oct 2009 & 358.1 & $4400-6200$ & 81 & $73 \mathrm{~S}$ & Slit & $32 / 118$ & 4 \\
\hline 7447A3 & 23 Oct 2009 & 358.1 & $4500-6300$ & 80 & $73 \mathrm{~S}$ & Slit & $32 / 118$ & 4 \\
\hline
\end{tabular}

443 Table 2 : List of SPICAM-UV observations used to study the Martian hydrogen corona during the Martian year 29. 444 The orbit number and sequence number of the observations are given in column 1. Column 3 gives the approximate 445 solar longitude Ls at the observation day. Columns 4, 5 and 6 refer to the tangent point of the line of sight. *For 446 these observations solar scattered light is observed at low altitudes on all bands polluting the airglow emissions. 
Only altitudes above $200 \mathrm{~km}$ are considered in these cases.

\section{References}

Bertaux, J-L., O. Korablev, S. Perrier, E. Quémerais, F. Montmessin, F. Leblanc, S. Lebonnois, P. Rannou, F. Lefevre, F. Forget, A. Fedorova, E. Dimarellis, A. Reberac, D. Fonteyn, J-Y. Chaufray, and S. Guibert, SPICAM on Mars Express : Observing modes and overview of UV spectrometer data and scientific results, J. Geophys. Res., 111, E10S90, doi:10.1029/2006JE002690, 2006

Bertucci, C., N. Romanelli, J-Y. Chaufray, D. Gomez, C. Mazelle, M. Delva, R. Modolo, F. Gonzalez-Galindo, and D.A. Brain, Temporal variability of waves at the proton cyclotron frequency upstream from Mars : Implication for Mars distant hydrogen exosphere, Geophys. Res. Lett., 40, 3809-3813, doi:10.1002/grl.50709, 2013

Beth, A., P. Garnier, D. Toublanc, I. Dandouras, and C. Mazelle, (2016), Theory for planetary exospheres : II Radiation pressure effect on exospheric density profiles, Icarus, 266, 423- 432

Bhattacharyya, D., J.T. Clarke, J-L. Bertaux, J-Y. Chaufray, M.Mayyasi, (2015), A strong seasonal dependence in the martian hydrogen exosphere, Geophys. Res. Lett., 42, 8678- 868

Bhattacharyya, D., J.T. Clarke, J-L. Bertaux, J-Y. Chaufray, and M. Mayyasi, (2017) Analysis and modeling of remote observations of the martian hydrogen exosphere, Icarus, 281, 264-280

Bibring, J-P., Y. Langevin, J. Mustard, F. Poulet, R. Arvidson, A. Gendrin, B. Gondet, N. Mangold, P. Pinet, F. Forget, and the OMEGA team, Global Mineralogical and Aqueous history of Mars derived from 
OMEGA/Mars Express data, Science, 312, 400-404, (2006)

Bishop, J., et al., Phyllosilicate diversity and past aqueous activity revealed at Mawrth Vallis, Mars, Science, 321, 830-833, doi : 10.1126/science.1159699, (2008)

Boqueho, V., and P-L. Blelly, Contributions of a multimoment multispecies approach in modeling planetary atmospheres: Example of Mars, J. Geophys. Res., 110, A01313, doi: 10.1029/2004JA010414, 2005

Brinkmann, R.T., Departures from Jeans' escape rate for H and He in the Earth's atmosphere, (1970), Planet. Space Sci., $18,449-478$

Chaffin, M.S., J-Y. Chaufray, I.A.F Stewart, F. Montmessin, N.M. Schneider, and J-L. Bertaux, (2014), Unexpected variability of Martian hydrogen escape, Geophys. Res. Lett., 41, 314- 320, doi: 10.1002/2013GL058578

Chaffin, M.S., J. Deighan, N.M. Schneider, and A.I.F. Stewart, Elevated atmospheric escape of atomic hydrogen from Mars induced by high-altitude water, Nat. Geosc., 10, 174-178, (2017)

Chaffin, M.S., J-Y. Chaufray, J. Deighan, N.M. Schneider, M. Mayyasi, J.T. Clarke, E. Thiemann, S.K. Jain, M.M.J. Crismani, A. Stiepen, F.G. Eparvier, W.E. McClintock, A.I.F. Stewart, G.M. Holsclaw, F. Montmessin, and B.M. Jakosky, Mars H escape rates derived from MAVEN/IUVS Lyman- $\alpha$ brightness measurements and their dependence on model assumptions, J. Geophys. Res: Planets, 123, 2192-2210, doi: 10.1029/2018JE005574, (2018)

Chaufray, J-Y., J-L. Bertaux, F. Leblanc, and E. Quémerais, (2008), Observation of the hydrogen corona with SPICAM on Mars Express, Icarus, 195, 598-613

Chaufray, J-Y., F. Leblanc, E. Quémerais, and J-L. Bertaux, Martian oxygen density at the exobase deduced from OI 130.4 nm observations by SPICAM on Mars Express, J. Geophys. Res., 114, E02006, doi:10.1029/2008JE003130, 2009

Chaufray, J-Y., J-L. Bertaux, E. Quémerais, E. Villard, F. Leblanc, Hydrogen density in the dayside venusian exosphere derived from Lyman- $\alpha$ observations by SPICAV on Venus Express, Icarus, 217, 767-778, 2012

Chaufray, J-Y., F. Gonzalez-Galindo, F. Forget, M.A. Lopez-Valverde, F. Leblanc, R. Modolo, and S. Hess, (2015a), Variability of the hydrogen in the martian upper atmosphere as simulated by a 3D atmosphere-exosphere coupling, Icarus, 245, 282-294

Chaufray, J-Y., J-L. Bertaux, F. Leblanc, E. Quémerais, and S. Sulis, (2015b), Observations of the nightside venusian hydrogen corona with SPICAV/VEX, Icarus, 262, 1-8 
Chaufray, J-Y., F. Gonzalez-Galindo, F. Forget, M.A. Lopez-Valverde, F. Leblanc, R. Modolo, and S. Hess, (2017), Reply to comment « On the hydrogen escape: Comment to Variability of the hydrogen in the Martian upper atmosphere as simulated by a 3D atmosphere-exosphere coupling by J-Y Chaufray et al." by V. Krasnopolsky, Icarus, 281, 262, doi : 10.1016/j.icarus.2017.07.013

Chaufray, J-Y., R.V. Yelle, F. Gonzalez-Galindo, F. Forget, M. Lopez-Valverde, F. Leblanc, R. Modolo, (2018), Effect of the lateral exospheric transport on the horizontal hydrogen distribution at the exobase of Mars, J. Geophys. Res., 123, 4241-4254, doi : 10.1002/2017JA025163

Clancy, R.T., B.J. Sandor, M.J. Wolff, P.R. Christensen, M.D. Smith, J.C. Pearl, B.J. Conrath, and R.J. Wilson, An intercomparison of ground-based millimeter, MGS TES, and Viking atmospheric temperature measurements: Seasonal and interannual variability of temperatures and dust loading in the global Mars atmosphere, J. Geophys. Res., 105, 9553-9571, 2000

Clarke, J. T., J-L. Bertaux, J-Y. Chaufray, G.R. Gladstone, E. Quémerais, J.K. Wilson, and D. Bhattacharyya, A rapid decrease of the hydrogen corona of Mars, Geophys. Res. Lett., 41, 8013-8020, 2014

Clarke, J.T., M. Mayyasi, D. Bhattacharyya, N.M. Schneider, W.E. McClintock, J.I. Deighan, A.I.F. Stewart, J-Y. Chaufray, M.S. Chaffin, S.K. Jain, A. Stiepen, M. Crismani, G.M. Holsclaw, F. Montmessin, and B. Jakosky, Variability of D and H in the Martian upper atmosphere observed with the MAVEN IUVS echelle channel, J. Geophys. Res., 122, 2336-2344, doi: 10.1002/2016JA023479

Emerich, C., P. Lemaire, J-C. Vial, W. Curdt, U. Schüle, K. Wilhelm, A new relation between the central spectral solar H I Lyman $\alpha$ irradiance and the line irradiance measured by SUMER/SOHO during the cycle 23., Icarus, $178,429-433,(2005)$

Fedorova, A., J-L. Bertaux, D.Betsis, F. Montmessin, O. Korablev, L. Maltagliati, and J. Clarke, Water vapor in the middle atmosphere of Mars during the 2007 global dust storm, Icarus, 300, 440, 2018

Forget, F., F. Hourdin, R. Fournier, C. Hourdin, O. Talagrand, M. Collins, S.R. Lewis, P.L. Read, J-P. Huot, Improved general circulation models of the Martian atmosphere from the surface to above $80 \mathrm{~km}$, J. Geophys. Res., 104, 24,155-24,175, 1999

Fox J.L., M. Benna, P.R. Mahaffy, and B.M. Jakosky, Water and water ions in the Martian thermosphere/ionosphere, Geophys. Res. Lett., 42, 8977-8985, doi: 10.1002/2015GL065465, (2015)

Gladstone, G.R., Auroral resonance line radiative transfer, J. Geophys. Res., 97, 1377-1387, 1992 
Gonzalez-Galindo, F., F. Forget, M.A. Lopez-Valverde, M. Angelats i Coll, and E. Millour, A ground-to-exosphere Martian general circulation model: 1 Seasonal, diurnal, and solar cycle variation of thermospheric temperatures, J. Geophys. Res., 114, E04001, doi:10.1029/2008JE003246, 2009

Gonzalez-Galindo, F., M.A. Lopez-Valverde, F. Forget, M. Garcia-Comas, E. Millour, and L. Montabone, (2015), Variability of the Martian thermosphere during eight Martian years as simulated by a ground-to-exosphere global circulation model, J. Geophys. Res., 120, 2020-2035, doi:10.1002/2015JE004925

Halekas, J. S. (2017), Seasonal variability of the hydrogen exosphere of Mars, J. Geophys. Res. Planets, 122, doi:10.1002/2017JE005306

Heavens et al, Hydrogen escape from Mars enhanced by deep convection in dust storms, Nature Astronom, 2, 126$132(2018)$

Hynek, B.M., M. Beach, and M.R.T. Hoke, Updated global map of Martian valley networks and implications for climate and hydrologic processes, J. Geophys. Res., 115, E09008, doi: 10.1029/2009JE003548, (2010)

Jakosky, B. et al., Loss of the Martian atmosphere to space: Present-day loss rates determined from MAVEN observations and integrated loss through time, Icarus, 315, 146-157, (2018)

Krasnopolsky, V., Mars' upper atmosphere and ionosphere at low, medium, and high solar activities: Implications for evolution of water, J. Geophys. Res., 107(E12), 5128, doi: 10.1029/2001JE001809, 2002

Krasnopolsky, V., Solar activity variations of thermospheric temperatures on Mars and a problem of CO in the lower atmosphere, Icarus, 207, 638-647, (2010)

Krasnopolsky, V., On the hydrogen escape from Mars: comments to "Variability of the hydrogen in the Martian upper atmosphere as simulated by a 3D atmosphere-exosphere coupling” by JY Chaufray et al., Icarus, 281, 262-263, (2017)

Krasnopolsky, V.A., Photochemistry of water in the martian thermosphere and its effect on hydrogen escape, Icarus, $321,62-70,2019$

Lasue, J., N. Mangold, E. Hauber, S. Clifford, W. Feldman, O. Gasnault, C. Grima, S. Maurice, and O. Mousis, Quantitative assessments of the Martian hydrosphere, Space Sci. Rev., 174, 155-212, (2013)

Leblanc, F., J-Y. Chaufray, J. Lilensten, O. Witasse, and J-L. Bertaux, Martian dayglow as seen by the SPICAM UV spectrograph on Mars Express, J. Geophys. Res., 111, E09S11, doi: 10.1029/2005JE002664, 2006

Lee, J.S., Refined Monte Carlo method for simulating angle-dependent partial frequency redistributions, Astrophys. 
J., 255, 303-306, 1982

Lopez-Valverde, M.A. et al., Investigation of the Mars upper atmosphere with ExoMars Trace Gas Orbiter, Space Sci. Rev., 214:29, doi: 10.1007/s11214-017-0463-4, (2018)

Maltagliati, L., F. Montmessin, O. Korablev, A. Fedorova, F. Forget, A. Määtänen, F. Lefèvre, and J-L. Bertaux, (2013) Annual survey of water vapor vertical distribution and water- aerosol coupling in the martian atmosphere observed by SPICAM/Mex solar occultations, Icarus, 223, 942-962

Mayyasi, M., D. Bhattacharyya, J. Clarke, A. Catalano, M. Benna, P. Mahaffy, E. Thiemann, C. Lee, J. Deighan, J. Sonal, and 9 co-authors, (2018), Significant space weather impact on the escape of hydrogen from Mars, Geophys. Res. Lett., 45, 8844

Montabone, L., F. Forget, E. Millour, R.J. Wilson, S.R. Lewis, B. Cantor, D. Kass, A. Kleinbohl, M.T. Lemmon, M.D. Smith, M.J. Wolff, Eight-year climatology of dust optical depth on Mars, Icarus, 251, 65-95, 2015

Montmessin, F., F. Forget, P. Rannou, M. Cabane, R. M. Haberle, Origin and role of water ice clouds in the Martian water cycle as inferred from a general circulation model, J. Geophys. Res., 109, E10004, doi : 10.1029/2004JE002284, 2004

Montmessin, F., O. Korablev, F. Lefevre, J-L. Bertaux, A. Fedorova, A. Trokhimovskiy, J-Y. Chaufray, G. Lacombe, A. Reberac, L. Maltagliati, Y. Willame, S. Guslyakova, J-C. Gerard, A. Stiepen, D. Fussen, N. Mateshvili, A. Maatanen, F. Forget, O. Witasse, F. Leblanc, A.C. Vandaele, E. Marcq, B. Sandel, B. Gonder, N. Schneirder, M Chaffin, N. Chapron, SPICAM on Mars Express: A 10 year in-depth survey of the Martian atmosphere, Icarus, 297, 195-216, 2017

Navarro, T., J.-B. Madeleine, F. Forget, A. Spiga, E. Millour, F. Montmessin, and A. Määttänen. Global climate modeling of the Martian water cycle with improved microphysics andadiatively active water ice clouds. Journal of Geophysical Research (Planets), 119:1479- 1495, 2014

Orosei, R., R.L. Jordan, D.D Morgan, M. Cartacci, A. Cicchetti, F. Duru, D.A. Gurnett, E. Heggy, D.L. Kirchner, R. Noschese, W. Kofman, A. Masdea, J-J. Plaut, R. Seu, T.R. Watters, G. Picardi, Mars Advanced Radar for subsurface and ionospheric sounding (MARSIS) after nine years of operation : A summary, Planet Space Sci., $112,98-114,(2015)$

Orosei, R., S.E. Lauro, E. Pettinelli, A. Ciccheti, M. Coradini, B. Cosciotti, F. Di Paolo, E. Flamini, E. Mattei, M. Pajola, and 12 co-auhors, Radar evidence of subglacial liquid water on Mars, Science, 361, 490, (2018) 
Quémerais, E., Angle dependent partial frequency redistribution in the interplanetary medium at Lyman- $\alpha$, Astron. Astrophys., 358, 353-367, 2000

Rahmati, A., D.E. Larson, T.E. Cravens, R.J. Lillis, J.S. Halekas, J.P. McFadden, D.L. Mitchell, E.M.B. Thiemann, J.E.P. Connerney, P.A. Dunn, C.O. Lee, F.G. Eparvier, G.A.DiBraccio, J.R. Espley, J.G. Luhmann, C. Mazelle, and B.M.. Jakosky, Seasonal variability of neutral escape from Mars as derived from MAVEN pickup ion observations, J. Geophys. Res. : Planets, 123, 1192-1202, 10.1029/2018JE005560, (2018)

Romanelli, N. C. Mazelle, J-Y. Chaufray, K. Meziane, L. Shan, S. Ruhunusiri, J.E.P. Connerney, J. R. Espley, F. Eparvier, T.E. Thiemann, et al., 2016, Proton cyclotron, waves occurrence rate upstream from Mars observed by MAVEN : Associated variability of the Martian upper atmosphere, J. Geophys. Res., 121, 11,113-11,128, doi : 10.1002/2016JA023270

Rottman, G.J., N.W. Thomas; W. McClintock, SORCE solar UV irradiance results, Adv. Space Sci., 37, 201-208, (2006)

Shaposhnikov, D.S., A.S. Medvedev, A.V. Rodin, and P. Hartogh, Seasonal water "pump" in the atmosphere of Mars: Vertical transport to the thermosphere, Geophys. Res. Lett., 46, 4161-4169, (2019)

Shizgal, B., and R. Blackmore, A collisional kinetic theory of a plane parallel evaporating planetary atmosphere, Planet. Space Sci., 34, 279-291, (1986).

Terada, K., N. Terada, H. Shinagawa, H. Fujiwara, Y. Kasaba, K. Seki, F. Leblanc, J-Y. Chaufray, and R. Modolo, A full-particle Martian upper thermosphere-exosphere model using the DSMC method, J. Geophys. Res., 121, 1429-1444, doi: 10.1002/2015JE004961, 2016

Vandaele, A.C, O. Korablev, F. Daerden, et al., Martian dust storm impact on atmospheric $\mathrm{H} 2 \mathrm{O}$ and D/H observed by ExoMars Trace Gas Orbiter, Nature, 568, 52, (2019)

Vidal-Madjar, A., and J-L. Bertaux, A calculated hydrogen distribution in the exosphere, Planet Space Sci., 20, 11471162,1972

Yamauchi, M., T. Hara, R. Lundin, E. Dubinin, A. Fedorov, J-A. Sauvaud, R.A. Frahm, R. Ramstad, Y. Futaana, M. Holmstrom, S. Barabash (2015), Seasonal variation of martian pick-up ions: Evidence of breathing exosphere, Planet. \& Sp. Sci., 119, p. 54 


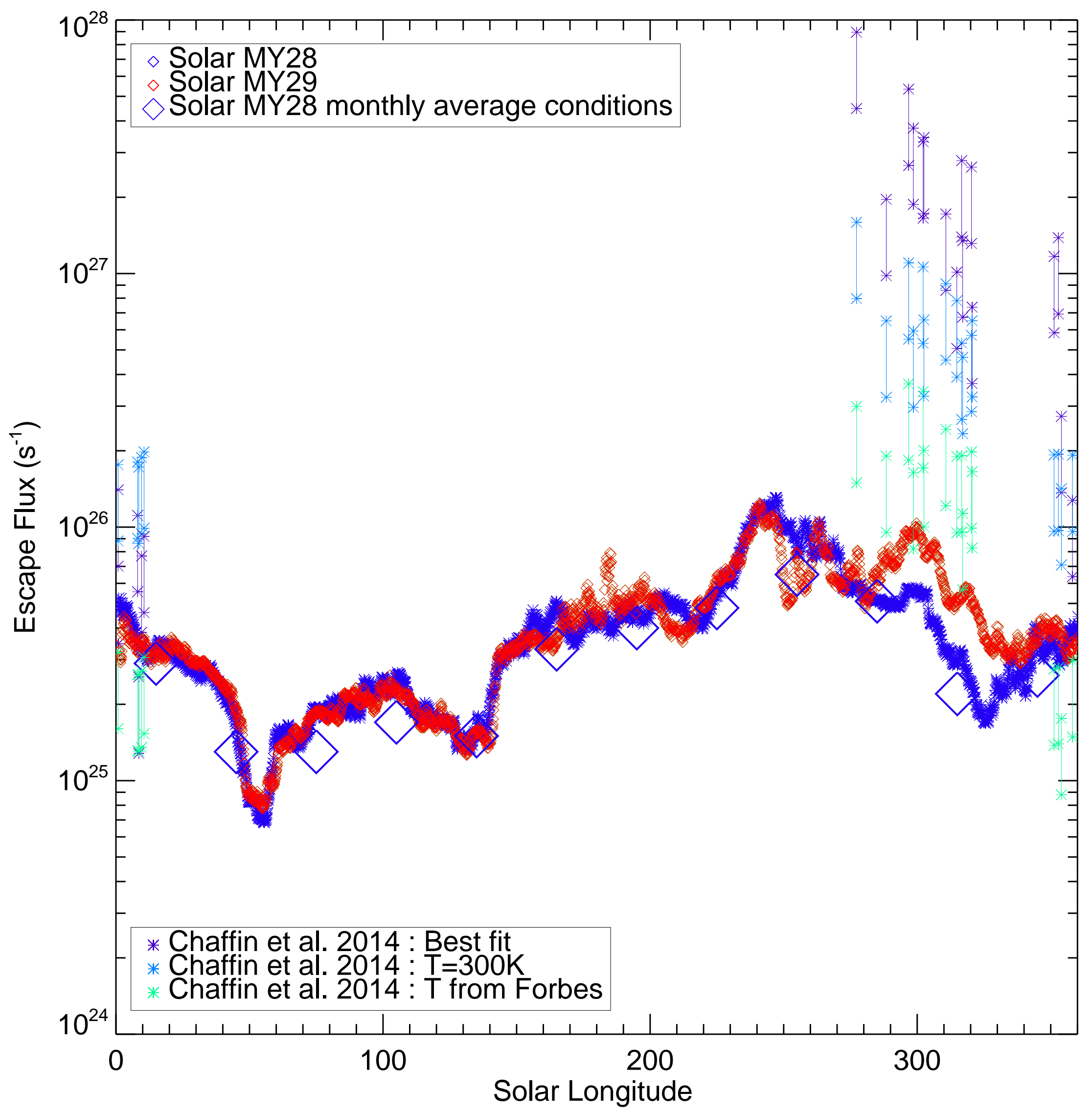


